RAFAEL SALLES

EXPERIMENTAL ANALYSIS OF FLUID-STRUCTURE INTERACTION PHENOMENA ON A VERTICAL FLEXIBLE CYLINDER: MODAL COEFFICIENTS AND PARAMETRIC RESONANCE 
RAFAEL SALLES

\section{EXPERIMENTAL ANALYSIS OF FLUID-STRUCTURE INTERACTION PHENOMENA ON A VERTICAL FLEXIBLE CYLINDER: MODAL COEFFICIENTS AND PARAMETRIC RESONANCE}

\footnotetext{
Dissertation presented to Escola Politécnica da Universidade de São Paulo in partial fulfillment of the requirements for the Master of Science degree
} 
RAFAEL SALLES

\title{
EXPERIMENTAL ANALYSIS OF FLUID-STRUCTURE INTERACTION PHENOMENA ON A VERTICAL FLEXIBLE CYLINDER: MODAL COEFFICIENTS AND PARAMETRIC RESONANCE
}

\author{
Dissertation presented to Escola Politécnica \\ da Universidade de São Paulo in partial \\ fulfillment of the requirements for the Master \\ of Science degree \\ Area: Mechanical Engineering \\ Advisor: Professor Doutor Celso Pupo Pesce
}

São Paulo 
Este exemplar foi revisado e corrigido em relação à versão original, sob responsabilidade única do autor e com a anuência de seu orientador.

São Paulo, de de

Assinatura do autor:

Assinatura do orientador:

Catalogação-na-publicação

Salles, Rafael

Experimental Analysis of Fluid-Structure Interaction Phenomena on a Vertical Flexible Cylinder: Modal Coefficients and Parametric Resonance / R. Salles -- versão corr. -- São Paulo, 2016.

$116 \mathrm{p}$.

Dissertação (Mestrado) - Escola Politécnica da Universidade de São Paulo. Departamento de Engenharia Mecânica.

1.Tubos flexíveis 2.Riser 3.Interação Fluido-Estrutura 4.Estrutura Offshore 5.Instabilidade de Mathieu I.Universidade de São Paulo. Escola Politécnica. Departamento de Engenharia Mecânica II.t. 


\section{Acknowledgements}

Primeiramente, eu preciso agradecer aos meus pais pela força e apoio durante toda a pós-graduação, afinal a chance de realizar este trabalho é fruto de capacidade e oportunidade. Creio que eles deram a mim ambas primorosamente. Além dos meus pais, posso citar o apoio das avós sempre muito queridas, bem como de outros familiares que sempre depositaram em mim esperança para dar-me forças de seguir meus sonhos.

Muito há o que agradecer ao meu orientador professor Celso Pesce. Um homem de visão que sempre viu em mim algo que ainda não consegui e, ao depositar sua fé em mim, deu-me oportunidades, oportunidade para ser orientado, para crescer, para trabalhar com outros tantos colegas geniais. Ainda assim, creio que o mais caro que pude receber dele foram palavras em momentos difíceis. Fora meus pais, acho que apenas Celso realmente acredita e deposita tua fé em mim tão sinceramente.

Não poderia deixar de agradecer minha terapeuta Carol porque, sem a ajuda dela, eu não teria conseguido sobreviver aos meus 20 -e-poucos até o presente momento. Os anos 2007 da Britney chega para todos.

Seguindo aos colegas, o primeiro não poderia deixar de ser o professor Guilherme Franzini por ter tanta paciência para conseguir acompanhar-me com passos muito mais lentos do que os dele. Aos colegas Caio e Luzia, apenas penso em vós como figuras angelicais que foram postas no meu dia-a-dia.

À equipa do LMO por estar presente em diversos momentos da minha vida acadêmica. Colegas de longa data como Marcos Rabelo e Flávia Milo que marcaram uma época muito importante da minha formação profissional. Aos colegas do TPN pela imensa ajuda e disponibilidade em momentos de dificuldade, meus sinceros agradecimentos, em especial aos Rodolfo Gonçalves e Pedro Melo por terem ficado comigo até os últimos minutos de 2014 para realizar meus ensaios com o modelo vertical no ar.

O pessoal que cuidou da parte administrativa, Larissa, Marta, Lúcia, saibam que eu devo muito a vós; à equipa técnica do LMO e TPN reservo meu agradecimento sincero pela paciência depositada em mim, que sou naturalmente uma pessoa atrapalhada; e especialmente a Mariza e Regiane do PPGEM por serem as pessoas mais solícitas que 
conheço.

Não poderia deixar de agradecer ao professor Raul Lima por sempre ter idéias e opiniões que foram muito importantes na minha formação nos últimos anos. Assim como ele, outros professores: Marcos Pimenta, Euryale Zerbini, Carlos Mazzilli e Roberto Ramos.

Por último deixo um grande < 3 INHAÍ < 3 para meus amigos de longa data, Lara, Bela, Bafominho, Bia, Esther, Ranily, Lurdes, Bucious e Bris; sou grato por todo o tempo que estão ao meu lado diretamente, indiretamente e carmicamente. Agradeço à Nat por ter-me como Moze by Ishikawa, assim como guardo as melhores lembranças dos novos amigos com quem a vida presenteou-me.

Agradecimento também ao $\mathrm{CNPq}$ (Conselho Nacional de Desenvolvimento Científico e Tecnológico), sua assessoria científica e seus funcionários pelo suporte financeiro e administrativo durante o período de vigência da minha bolsa de mestrado (processo 131549/2013-8). 
Dedication

I dedicate this work to my grand parents, specially to my grandmother Mainha who will always be missed. 


\section{Epigraph}

"Les découvertes des satellites de Jupiter, des phases de Vénus, des taches du Soleil, etc., ne demandaient que des télescopes et de l'assiduité; mais il fallait un génie extraordinaire pour démêler les lois de la nature dans des phénomènes que l'on avait toujours eus sous les yeux, mais dont l'explication avait néanmoins toujours échappé aux recherches des Philosophes."

J.-L. Lagrange (1736 - 1813), Sur les différents Principes de la Dynamique, Mécanique Analytique - Seconde Partie. 


\begin{abstract}
Oil and gas exploitation in deep waters has become more than just a profit business to be a daily necessity, since the world energy matrix is based on fossil components. Risers are offshore structures that are intimately linked with oil and gas exploitation and those are subjected to a great variety of effects in field, e.g., marine currents, Vortex Induced Vibration (VIV), heave motion caused by gravitational waves, non-linear contact with the sea floor, and many others. Riser dynamics is essentially non-linear and experimental tests in real scale are almost impossible due to a great variety of control parameters acting concomitantly. Small-scale models are a better experimental approach. Nevertheless, there are many structural and hydrodynamical parameters to be evaluated. Considering only vertical risers in the present work, Galerkin's modal decomposition is used in order to reduce the dynamics of a vertical flexible cylinder to a few linear modes in which the majority of energy and information are contained. From the modal analysis, added mass and structural parameters damping of a vertical flexible cylinder using data obtained from free-decay tests performed both in water and in air are evaluated. Finally, a modal Mathieu-Hill oscillator with non-linear damping is constructed and, based on a Strutt diagram, modal stability under parametric resonance is discussed.
\end{abstract}

Keywords: Flexible pipes, Riser, Fluid-structure interaction, Offshore structure, Mathieu instability. 


\section{Resumo}

Exploração de óleo e gás em bacias de águas profundas tem-se tornado mais do que apenas uma economia lucrativa, para ser uma necessidade diária, já que a matriz energética mundial está baseada em componentes fósseis. Risers são estruturas offshore ligadas intimamente com a exploração de óleo e gás e essas estão sujeitas a uma grande variedade de efeitos na operação, e.g., correntes marítimas, Vibrações Induzidas por Vórtices (VIV), movimento de heave causado por ondas gravitacionais, contato não-linear com o solo marinho, entre outros. Dinâmica de risers é essencialmente não-linear e testes experimentais em escala real são praticamente impossíveis devido a uma enorme variedade de parâmetros de controle agindo concomitantemente. Modelos em escala reduzida são uma abordagem experimental mais conveniente. Não obstante, há muitos parâmetros estruturais e hidrodinâmicos a serem determinados. Considerando apenas risers verticais no trabalho presente, a decomposição modal de Galerkin é usada a fim de reduzir a dinâmica de um cilindro fléxivel vertical a alguns modos lineares em que a maior parte da energia e informação estão contidos. A partir da análise modal, parâmetros de massa adicional e amortecimento estrutural de um cilindro flexível vertical são obtidos usando testes de decaimento livre conduzidos na água e no ar. Finalmente, um oscilador modal de Mathieu-Hill com amortecimento não-linear é proposto e, baseado em um diagrama de Strutt, a estabilidade modal sob excitação de ressonânica paramétrica é discutida.

Palavras-chave: Tubos flexíveis, Riser, Interação fluido-estrutura, Estrutura offshore, Instabilidade de Mathieu. 



\section{List of Figures}

1.1 Typical riser configurations: vertical and SCR . . . . . . . . . . . . . . . . . . . . . . 1

1.2 Plataform and other ocean units on which risers might be used. . . . . . . . . . . 2

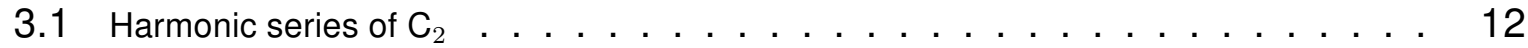

3.2 Pythagorean relationship between frequency and wavelength in a ideal vibranting string 13

3.3 Schematic model of a classical vibrating string bi-articulated with constant distributed mass, $\mu$, and constant traction,.$\ldots \ldots \ldots \ldots$

3.4 Vertical tensioned string subjected to its own weight $\ldots \ldots \ldots$

3.5 Sine and Bessel modes: 1st-to-4th mode. . . . . . . . . . . . . . . . . . . . 18

3.6 Free-decay test in water on the experimental model. Power spectrum of the transversal movement amplitude along the model spanwise . . . . . . . . . . . . . . . 20

4.1 A segment of the "as built" model: a silicon tube filled with stainless steel micro-spheres. 29

4.2 Viscous-elastic behavior of experimental model under cyclic tension test. Maximum engineering deformation of $\epsilon \approx 0.1 \%$ at $f=1.5 \mathrm{~Hz} \ldots \ldots \ldots 30$

4.3 Vertical model. Note: reflexive targets attached to the model in order to capture its displacement. . . . . . . . . . . . . . . . . . . 32

4.4 Submerged optical cameras: measuring displacement. Note: model launched in "catenary" configuration. . . . . . . . . . . . . . . . . . . . 32

4.5 Servo driver and load cell with the model in vertical configuration at IPT. . . . . . . . 33

4.6 Vertical model. Note: reflexive targets attached to the model in order to capture its displacement. . . . . . . . . . . . . . . . . . . . . 34

4.7 schematic experimental set-up. . . . . . . . . . . . . . . . . . . . . . . 34

4.8 Experimental model used in air. Unstretched length, $L_{0}=2613 \mathrm{~mm} \ldots \ldots \ldots$

4.9 Experimental model used in air. Detail to the discretization of the model using optical targets and the filling of micro-spheres. . . . . . . . . . . . . . 
4.10 Superior part of the structure assembled and base in which the model is fixed. . . . 36

4.11 Lateral view of the structure. . . . . . . . . . . . . . . . . . . . . 36

4.12 The final structure with model suspended. . . . . . . . . . . . . . . . . . 37

4.13 Set of optical tracking cameras positioned in front of the model. . . . . . . . . . . . 37

4.14 The final structure assembled with model suspended further detailed. . . . . . . . . 38

5.1 Scalograms of imposed heave $-f_{t}: f_{N, 1}=1: 1 \ldots \ldots \ldots \ldots$

5.2 PSD of imposed heave $-f_{t}: f_{N, 1}=1: 1 \ldots \ldots \ldots \ldots \ldots$

5.3 PSD of imposed heave $-f_{t}: f_{N, 1}=3: 1 \ldots \ldots \ldots \ldots \ldots \ldots$. . . . . . . . 42

5.4 Scalograms and PSD spectra from imposed heave $-f_{t}: f_{N, 1}=2: 1 \ldots \ldots$. . . . 43

5.5 Modal representation using three harmonics. Experimental data and Bessel-type eigenfunction base. VIV $-V_{R, 1}=6.00 . \ldots \ldots \ldots \ldots$. . . . . . . . . 45

5.6 Modal representation using three harmonics. Experimental data and sine eigenfunction base. Heave $-f_{t}: f_{N, 1}=2: 1 \ldots \ldots \ldots \ldots$

5.7 Modal representation using three harmonics. Experimental data and sine eigenfunction base. Heave $-f_{t}: f_{N, 1}=3: 1 \ldots \ldots \ldots \ldots \ldots \ldots$

5.8 Modal representation using four harmonics. Experimental data and sine eigenfunction base. Heave $-f_{t}: f_{N, 1}=3: 1 \ldots \ldots \ldots \ldots$

5.9 Free-Decay Test - Initial impulse position along spanwise. Impulse A - Test 4; Impulse B - Test 5 ; and Impulse $\mathrm{C}-$ Test $6 \ldots \ldots \ldots$. . . . . . . . . . . . . 48

5.10 Free-Decay Test - Time Series of the Target $25 \ldots \ldots \ldots$

5.11 Free-Decay Test - Amplitude scalogram $\left(y^{\star}\right) \ldots \ldots \ldots$

5.12 Free-Decay - Amplitude Spectrum Density along spanwise: Test 4 . . . . . . . . . . 51

5.13 Free-Decay - Amplitude Spectrum Density along spanwise: Test $5 \ldots \ldots 1$

5.14 Free-Decay - Amplitude Spectrum Density along spanwise: Test 6 . . . . . . . . 52

5.15 Free-Decay Test - Modal Amplitude Time Series. . . . . . . . . . . . . . . . . . 52

5.16 Free-Decay Test $4-$ Amplitude spectrogram $\left(y^{\star}\right) \ldots \ldots \ldots$

5.17 Free-Decay Test - Modal Amplitude Time Series. . . . . . . . . . . . . . . . 55

5.18 Free-Decay Test $4-$ Modal linear damping: Mode $1 \ldots \ldots$. . . . . . . . . . . . 55

5.19 Free-Decay Test - Modal Amplitude Time Series. . . . . . . . . . . . . . . . . 56

5.20 Free-Decay Test $4-$ Modal linear damping: Mode $2 \ldots \ldots$. . . . . . . . . . . . 56

5.21 Free-Decay Test -Modal Amplitude Time Series. . . . . . . . . . . . . . . . . 57

5.22 Free-Decay Test $4-$ Modal linear damping: Mode $3 \ldots \ldots$. . . . . . . . . . . . . . 57 
5.23 Free-Decay Test $5-$ Amplitude spectrogram $\left(y^{\star}\right) . \ldots \ldots$. . . . . . . . 58

5.24 Free-Decay Test $5-$ Modal linear damping: Mode 1. . . . . . . . . . . . . . . . . 59

5.25 Free-Decay Test $5-$ Modal linear damping: Mode $2 \ldots \ldots$. . . . . . . . . . . . . 59

5.26 Free-Decay Test $5-$ Modal linear damping: Mode $3 . \ldots$. . . . . . . . . . . . 60

5.27 Free-Decay Test 6 - Amplitude spectrogram $\left(y^{\star}\right)$. . . . . . . . . . . . . . 61

5.28 Free-Decay Test 6 - Modal linear damping: Mode 1 . . . . . . . . . . . . . . 62

5.29 Free-Decay Test 6 - Modal linear damping: Mode $2 \ldots \ldots$. . . . . . . . . . . . . . 62

5.30 Free-Decay Test 6 - Modal linear damping: Mode 3 . . . . . . . . . . . . . . 63

5.31 Free-Decay Test - Tension imposed at the top. . . . . . . . . . . . . . . 65

5.32 Hydrodynamics coefficients vs. Keulegan-Carpenter number for constant values of the frequency parameter and Reynolds number . . . . . . . . . . . . . . . . 68

5.33 Normalized amplitude of free-decay test in water . . . . . . . . . . . . . . 70

5.34 Second bifurcation on Strutt diagram by introducing a quadratically damping term. . . 72

5.35 Strutt diagram of classical undamped Mathieu-Hill oscillator. . . . . . . . . . . . . 73

5.36 Modal Strutt diagrams approximated to a Mathieu oscillator with quadratically damping function. Heave: Red square $-f_{t}: f_{N, 1}=1: 1$; Green square $-f_{t}: f_{N, 1}=2: 1 ;$ \& Blue square $-f_{t}: f_{N, 1}=3: 1 \ldots \ldots \ldots \ldots 74$

5.37 Amplitude series, frequency spectra (FFT) and phase portraits for the first mode. . . . 78

5.38 Amplitude series, frequency spectra (FFT) and phase portraits for the second mode. . 79

5.39 Amplitude series, frequency spectra (FFT) and phase portraits for the third mode. . . 80 


\section{List of Tables}

4.1 Dimensionless parameters. . . . . . . . . . . . . . . . . . . . . . . . . . . 28

4.2 Quantities feautures and Froude similarity. . . . . . . . . . . . . . . . . 28

4.3 Data summary: SCR, scaled model, designed small-scale model and "as built". . . . 29

4.4 Mechanical properties from the experimental model of flexible vertical cylinder model in water. . . . . . . . . . . . . . . . . . . . . 31

4.5 Mechanical properties from the experimental model of flexible vertical cylinder model in air. . . . . . . . . . . . . . . . . . . . 38

5.1 Free-decay: eigenfrequencies and linear damping results - Tests: 4,5 and 6 . . . 63

5.2 Mean values of frequency and linear damping considering the most suitable results from Tests 4,5 and $6 \ldots \ldots \ldots \ldots \ldots$

5.3 Modal parameters eq. 3.30 analytical expressions, using sine eigenfunction base. . . 64

5.4 Axial rigidity equivalence . . . . . . . . . . . . . . . . . . 66

5.5 Modal added mass . . . . . . . . . . . . . . . . . . 67

5.6 Modal Strutt diagram stability of the trivial solution for experiments in water $\ldots . . .75$

5.7 Parameters of Mathieu-Hill oscillators (ROMs) studied in Franzini et al. (2015) . . . . 75

5.8 Frequency ratios of periodic response. Prediction by Strutt diagram versus determined from spectral analysis. . . . . . . . . . . . . . . . . . . . . 77 


\section{List of Abbreviations}

Institutions

CNPq National Counsel of Technological and Scientific Development

FAPESP São Paulo Research Foundation

FINEP Financiadora de Estudos e Projetos

IPT Institute for Technological Research

LIFE\&MO Laboratory of Fluid-Structure Interaction \& Offshore Mechanics

NDF $\quad$ Fluid \& Dynamics Research Group

LMO Laboratory of Offshore Mechanics

TPN Numerical Offshore Tank

USP São Paulo University

Other abbreviations

$\mathrm{CH}-\mathrm{TPN} \quad$ Hydrodynamic Calibrator

CFD Computational Fluid Dynamics

FEM Finite Element Method

FFT Fast Fourier Transform

GT Gabor Transform

HHT Hilbert-Huang Transform

JTFA Joint Time-Frequency Analysis

PVW Principle of Virtual Work

R\&D Research \& Development

SCR Steel Catenary Riser

SM Spectral Methods

STFT Short Time Fourier Transform

VIM Vortex-Induced Motion

VIV Vortex-Induced Vibration

VSIV Vortex Self Induced Vibration

WT Wavelet Transform 


\section{List of Symbols}

\section{Latin letters}

\begin{tabular}{|c|c|}
\hline$A(t)$ & Amplitude temporal series \\
\hline$A_{n}$ & Modal temporal amplitude \\
\hline$A_{t}$ & Amplitude parametrically imposed at the top \\
\hline$A^{\star} x_{n}$ & Dimensionless in-line modal amplitude of th \\
\hline$A^{\star} y_{n}$ & $\begin{array}{l}\text { Dimensionless transversal modal amplitu } \\
A y_{n} / D\end{array}$ \\
\hline$a_{n}, \tilde{a}_{n}$ & Modal added mass coefficient, $=m_{a} / \mu$ \\
\hline$C_{n}, C_{1}, C_{2}$ & Coefficients of formula deduction \\
\hline$C_{a, n}$ & Modal added mass coefficient, $=m_{a} / m_{d}$ \\
\hline$C_{D}$ & Drag coefficient \\
\hline$C_{m}$ & Inertia coefficient, $=C_{a}+1$ \\
\hline$C_{n}$ & Modal quadratically damping \\
\hline$C_{s, n}$ & Modal linear damping \\
\hline$c_{s}$ & Structural linear damping, $=T / \mu$ \\
\hline$c_{0}$ & Wave celerity \\
\hline$D$ & Diameter, cylinder diameter \\
\hline$E A$ & Axial stiffness \\
\hline$E I$ & Bending stiffness \\
\hline$f$ & Frequency \\
\hline$f_{N, n}$ & Natural frequency related to the $n$-th mode \\
\hline$F_{s}$ & Sampling frequency \\
\hline$f_{s}^{\star}$ & Reduced shedding frequency $=S t V_{R}$ \\
\hline$F r$ & Froude number $=U / \sqrt{g L}$ \\
\hline$g$ & Gravity \\
\hline$I$ & Interval, $=[0,1]$ \\
\hline$K_{a}$ & Axial stiffness, nondimensional $=E A / T$ \\
\hline$K_{f}$ & Bending stiffness, nondimensional $=\lambda_{f} / L$ \\
\hline$K C$ & Keulegan-Carpenter number $=2 \pi A / D$ \\
\hline$L$ & Stretched length \\
\hline$L_{0}$ & Unstretched length \\
\hline$L_{i}$ & Immersed length \\
\hline
\end{tabular}




$\begin{array}{ll}\mu & \text { Mass, cylinder mass } \\ M_{n} & \text { Modal total mass } \\ M_{n}^{a} & \text { Modal added mass, }=M_{n}^{s t r u c}+M_{n}^{a} \\ M_{n}^{d} & \text { Modal displaced mass } \\ M_{n}^{\text {struc }} & \text { Modal structural mass } \\ m_{a} & \text { Added mass, }=C_{a} m_{d}=a \mu \\ m_{d} & \text { Displaced mass }=\pi \rho_{w} D^{2} / 4 \\ m_{t} & \text { Total mass, } \mu+m_{a} \\ m^{\star} & \text { Mass ratio parameter }=\mu / m_{d} \\ R e & \text { Reynolds number }=U D / \nu \\ S t & \text { Strouhal number }=f_{v} D / U \\ t & \text { Time } \\ T & \text { Traction load on the cylinder } \\ T_{0} & \text { Traction load at the bottom of the cylinder } \\ T_{t} & \text { Static tension at the top of the cylinder } \\ U & \text { Stream induced velocity } \\ u(z, t) & \text { Displacement vector } \\ u^{x}(z, t), u^{y}(z, t) & \text { Displacement component in-line and transversal directions, } \\ w(z) & \text { respectively } \\ \hat{z} & \text { Weighting function } \\ V_{R, 1} & \text { Normalized axial position, }=z / L \\ x, y, z & \text { Reduced velocity of the first mode }=U / f_{N, 1} D \\ x^{\star}, y^{\star}, z^{\star} & \text { In-flow, cross-flow and axial cylinder displacements } \\ \hat{z} & \text { Nondimensional cylinder displacements, }=x / D,=y / D \text { and }=z / D \\ & \text { Normalized axial cylinder displacement, }=z / L \\ & \end{array}$

\section{Greek letters}

$\begin{array}{ll}\beta_{n} & \text { Modal quadratically damping ratio, }=C_{n} / M_{n}^{a} \\ \hat{\beta}_{n} & \text { Modal quadratically damping ratio, }=\beta_{n} / 2 \\ \tilde{\beta} & \text { Diameter, [mm] } \\ \beta_{K C} & \text { Keulegan-Carpenter frequency parameter } \\ \gamma & \text { Total distributed weight }=\mu g \\ \gamma_{i} & \text { Immersed distributed weight (with buoyancy effect) }=\mu g-\pi \rho_{w} D^{2} L_{i} g / 4\end{array}$




\begin{tabular}{|c|c|}
\hline$\delta_{n}$ & Dimensionless frequency parameter, $=2 \omega_{N, n} / \omega_{t}$ \\
\hline$\delta_{n}^{\star}$ & Dimensionless frequency parameter, $=\delta_{n}-\left(\zeta_{n}^{\star} \omega_{N, n}\right)^{2}$ \\
\hline$\epsilon$ & Deformation, nondimensional [\%] \\
\hline$\varepsilon_{n}$ & Dimensionless modulation, $=2 \hat{\xi}_{n} /\left(\omega_{t}\right)^{2}$ \\
\hline$\zeta_{n}$ & Modal nondimensional linear damping ratio $=C_{s, n} /\left[2 M_{n} \omega_{N}\right][\%]$ \\
\hline$\zeta_{n}^{\star}$ & Modal linear damping ratio $=2 \zeta_{n} / \omega_{t}$ \\
\hline$\tilde{\zeta}$ & Deduction coefficient for Bessel modes \\
\hline$\eta_{n}$ & Modal stiffness related to the $n$-th mode \\
\hline$\theta^{n}$ & Modal angle phase related to the $n$-th mode \\
\hline$\vartheta^{n}(t)$ & $\begin{array}{l}\text { Modal amplitude temporal series (change of variables) related to the } \\
n \text {-th mode }\end{array}$ \\
\hline$\lambda$ & Wavelength \\
\hline$\lambda_{F r}$ & Froude scale parameter \\
\hline$\lambda_{f}$ & Flexural length \\
\hline$\mu$ & Distributed mass $=m / L$ \\
\hline$\nu$ & Kinematic viscosity \\
\hline$\xi_{n}$ & Modal stiffness modulation related to the $n$-th mode \\
\hline$\hat{\xi}_{n}$ & Modal stiffness modulation related to the $n$-th mode, $\xi_{n} / M_{n}$ \\
\hline$\tilde{\xi}_{n}$ & Deduction coefficient for Bessel modes \\
\hline$\rho_{w}$ & Water specific mass \\
\hline$\tau$ & Dimensionless time, $=\omega_{t} t / 2$ \\
\hline$\varphi^{n}(t)$ & Modal temporal amplitude series \\
\hline$\phi$ & Phase angle \\
\hline$\psi$ & Eigenfunction, modal shape \\
\hline$\Omega$ & Frequency $[\mathrm{rad} / \mathrm{s}]$ \\
\hline$\omega_{n}$ & Frequency of $n$-th mode $[\mathrm{rad} / \mathrm{s}]$ \\
\hline$\omega_{N, n}$ & Eigenfrequency, natural frequency of $n$-th mode $[\mathrm{rad} / \mathrm{s}]$ \\
\hline
\end{tabular}

Other symbols

$\begin{array}{ll}b & \text { Flat } \\ \mathfrak{F} & \text { Fourier transform } \\ \mathfrak{I} & \text { Imaginary part of a complex number }\end{array}$




$\begin{array}{ll}\mathbb{N} & \text { Natural numbers } \\ \varnothing & \text { Diameter, }[\mathrm{mm}] \\ \mathcal{O} & \text { Power order } \\ \mathbb{R} & \text { Real numbers } \\ \mathfrak{R} & \text { Real part of a complex number } \\ \sharp & \text { Sharp }\end{array}$




\section{Contents}

List of Figures . . . . . . . . . . . . . . . . . . . . . . . . . . . xiii

List of Tables . . . . . . . . . . . . . . . . . . . . . . . xiv

List of Abbreviations . . . . . . . . . . . . . . . . . . . . . . . . . . . . . . $\quad x v$

List of Symbols . . . . . . . . . . . . . . . . . . . . . . . . . . . . . . . . . . . $x$ xvi

Preface ......................... . . . . . . . . .

1 Introduction 1

2 Literature review 5

2.1 Approaches to modeling and experimental analysis . . . . . . . . . . . 8

2.2 Signal analysis techniques . . . . . . . . . . . . . . . . 9

3 Theoretical background 11

3.1 Modal mathematical formulation . . . . . . . . . . . . . . . . . . . 11

3.1.1 The classical vibrating string . . . . . . . . . . . . . . . . . 11

3.1 .2 Vertical string . . . . . . . . . . . . . . . . . . . . 14

3.1.3 Modal decomposition . . . . . . . . . . . . . . . . . . . 19

3.2 Modal oscillator . . . . . . . . . . . . . . . . . . . . . . 23

3.2.1 Mathematical formulation from the continuum . . . . . . . . . 23

4 Experimental Campaign 27

4.1 Experiments in water . . . . . . . . . . . . . . . . . . . . . . . 27

4.1 .1 Froude similitude . . . . . . . . . . . . . . . . . . . . 27

4.1.2 Experimental set-up: a brief description . . . . . . . . . . . . 31

4.2 New experiments in air . . . . . . . . . . . . . . . . . . . 35

5 Experimental Analysis: methodology and results 39

5.1 Modal representation . . . . . . . . . . . . . . . . . . . . . . 39

5.1.1 Imposed heave motion at the top . . . . . . . . . . . . . . . . . . 39

5.2 Free-Decay Test in air . . . . . . . . . . . . . . . . . . . . . . . . . 48

5.2.1 Modal Structural Damping Coefficient . . . . . . . . . . . . . 48

5.2 .2 Modal Added Mass . . . . . . . . . . . . . . . . . . . . . . . 64

5.3 Parametric resonance and Mathieu instability . . . . . . . . . . . . . . 71

6 Conclusions $\quad 81$

$\begin{array}{ll}\text { References } & 93\end{array}$ 


\section{Preface}

The Laboratory of Offshore Mechanics (LMO) is a large group that assembles a few research groups, among them the Laboratory of Fluid-Structure Interaction \& Offshore Mechanics (LIFE\&MO). The LIFE\&MO has dedicated its lifetime to study fluid-structure interaction phenomena and many aspects of offshore mechanics since 1990. Such themes include structural mechanics of risers, pipes and mooring lines, dynamic positioning of floating stations, non-linear dynamics and hydrodynamics. Its main subject is Offshore Engineering in deep water, patronized by institutions such as: Prysmian, Petrobras, FAPESP, CNPq and FINEP. Some pioneering works developed by the LMO group culminated with, just to cite a few:

- The Steel Catenary Riser (SCR) draft which, pioneering, was installed on a semi-submersible platform (the P-18), see Pesce et al. (1994);

- A first methodology establishment for design of a SCR, see (Pesce et al., 1996);

- The analytical treatment of riser dynamics, see Pesce (1997), Aranha et al. (1997), Pesce and Fujarra (1997), Fujarra et al. (1997), Pesce et al. (1998), Pesce et al. (1999), Ramos Jr. and Pesce (2003), Pesce et al. (2006a) and Silveira et al. (2007).;

Concerning experimental activities, LMO and LIFE\&MO have been pioneers at using several new monitoring techniques for VIV in flexible cylinders and riser models since 1997. Among those techniques, some uses accelerometry and conventional extensometry installed and distributed along the tested cylinder length; for further details, e.g., Fujarra (1997), Fujarra et al. (1998), Pesce and Fujarra (2000), Fujarra et al. (2001) and Fujarra (2002). The aforementioned experimental tests were performed at facilities, such as: Institute for Technological Research (IPT), the Michigan University and the Cornell University. The distributed extensometry technique were considered an innovation within the international community, as references such as Pesce and Fujarra (2000) and Fujarra et al. (2001) are acknowledged on important papers on VIV; see Williamson and Govardhan (2004). Those techniques are still used nowadays at LMO, e.g., Franzini et al. (2008), Franzini et al. (2009) and Franzini et al. (2011a), and other research groups, e.g., Morooka et al. (2009).

The LMO was also internationally pioneer using the Hilbert-Huang Transform (HHT) in order to analyze non-linear phenomena such as VIV; see Pesce et al. (2006), Silveira et al. (2007), Franzini et al. (2008), Franzini et al. (2010), Franzini et al. (2011a) and Franzini et al. (2011b). The HHT is a technique in which it is possible to obtain a time-frequency spectral analysis from extremely non-ergodic signals. This signal analysis tool was successfully employed on studies of offshore platforms Vortex-Induced Motion (VIM) phenomena; see Gonçalves et al. (2012).

Hereupon, the R\&D project entitled "Non-Linear Dynamics of Risers: non-linear interactions of hydro-elastic and contact nature"1 was contracted to Universidade de São Paulo (USP) by Petrobras in late 2009, under the Offshore Structures Thematic Network. Its scope is based on four complementary activities, as follows:

1. Mathematical modeling of risers dynamics with reduction techniques by using non-linear modes representation;

${ }^{1}$ The original title is in Portuguese: "Dinâmica não-linear de Risers: Interações não-lineares de natureza hidro-elástica e de contato". 
2. Parametric excitations and internal resonant responses, arising from dynamical interaction in several time and space scales, between heave imposed the top and Vortex-Induced Vibration (VIV);

3. Analysis of typical cases, focusing on non-linear dynamics of risers;

4. Experimental dynamics tests with riser small-scale model.

It is worthy mentioning that the "Non-Linear Dynamics of Risers" project has enabled the LIFE\&MO to advance on state-of-art researches on VIV and riser dynamics, improving technical and scientific topics related to theoretical models, modeling and monitoring techniques, as well as on several experimental analysis techniques applied to tests with small-scale riser models with catenary-shape and vertical configurations. It might be remarked the following advances:

1. Small-scale modeling and monitoring techniques

(a) Development of a new methodology for designing a small-scale riser models in which a silicon tube is filled with stainless steel micro-spheres, providing a geometrical, structural and dynamical hydro-elastic Froude similitude with a riser in real scale; see LIFE\&MO (2012b) and Pereira et al. (2012);

(b) Development and consolidation of displacement measurement technique along the model span, using an optical tracking system of cameras placed on air and immersed at different laboratory facilities: a Water Channel (NDF), a Hydrodynamic Calibrator (CH-TPN), and a Towing Tank (IPT); further details on LIFE\&MO (2012a, 2012b), Gonçalves et al. (2012), Franzini et al. (2013) and Pereira et al. (2013).

2. Experimental analysis techniques

(a) Consolidation of an analysis methodology in which conventional techniques are merged, such as: statistical methods, spectrum analysis (energy spectrum along model span) and time-domain analysis (amplitude-time scalograms); see LIFE\&MO (2012c, 2012d);

(b) Crystallization of the time-frequency spectrum analysis using the HHT; LIFE\&MO (2012b), Gonçalves et al. (2012), Franzini et al. (2013), Pereira et al. (2013) Franzini et al. (2015) and Franzini et al. (2016);

(c) Development of a modal decomposition method in VIV studies in flexible cylinders; as it may be regarded in LIFE\&MO (2012d) and Franzini et al. (2014).

Among those advances enumerated before, the particular design of a small-scale experimental model based on a silicon tube filled with stainless steel micro-spheres might be pointed out as a great innovation on the experimental field. The author had the chance of a life time to be part of the group who developed such experimental method, being able to collaborate actively; see Pereira et al. (2012).

All work carried out on the aforementioned project might be summed up on the series of technical reports LIFE\&MO (2011a, 2011b, 2011c, 2011d, 2011e, 2011f, 2012a, 2012b, 2012c, 2012d) and a colossal and unique amount of experimental data, including several appendices. An interesting note might be made, quoting the preface from LIFE\&MO (2012d), which says: 
The specific results obtained [on the technical reports LIFE\&MO (2012c, 2012d)] [...] are unique, since they came from a thorough planning and testing which were conducted systematically with a great degree of comprehensiveness and completeness.

The reader will be able to verify that the huge experimental data base [...] will serve not only to technical and scientific insights of experimental nature, however primarily to verification of theoretical models, whether numerical or analytical, being able to become an important source of benchmarking to industry, with consequent impact assessments on risers design meyhodology. (LIFE\&MO, 2012d, p. 12, own translation)

It is possible to affirm that the regarded R\&D project is unique and its large experimental data base will provide bright scientific insight for the next years. At least, that is what it is hoped. 


\section{Chapter 1}

\section{Introduction}

The dynamics of risers is eminently of nonlinear character, since it deals with vibration phenomena on large aspect ratio, $L / D \gg 100$, flexible cylindrical structures, associated with interactions with the fluid medium surrounding them and the combined support excitations and internal resonances of parametric nature. The complexity of these concurrent interactions - associated with its practical relevance in ocean engineering - makes the research topic in screen to remain in full development, both nationally and internationally, as evidenced by the long list of publications in the field, which forms part of Chapter 2.

Figure 1.1: Typical riser configurations: vertical and SCR.

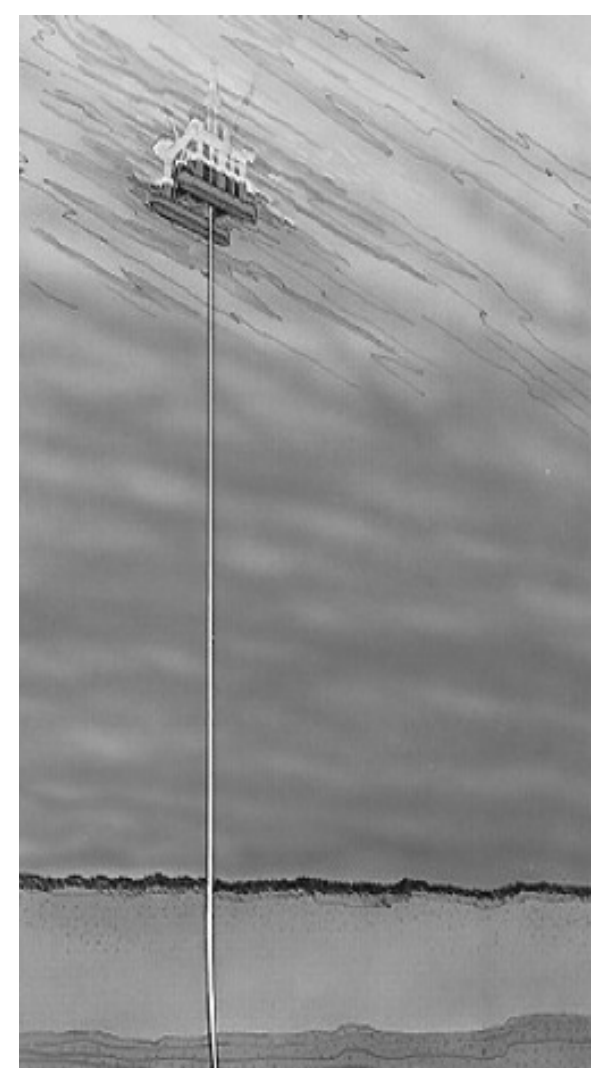

(a) Vertical riser.

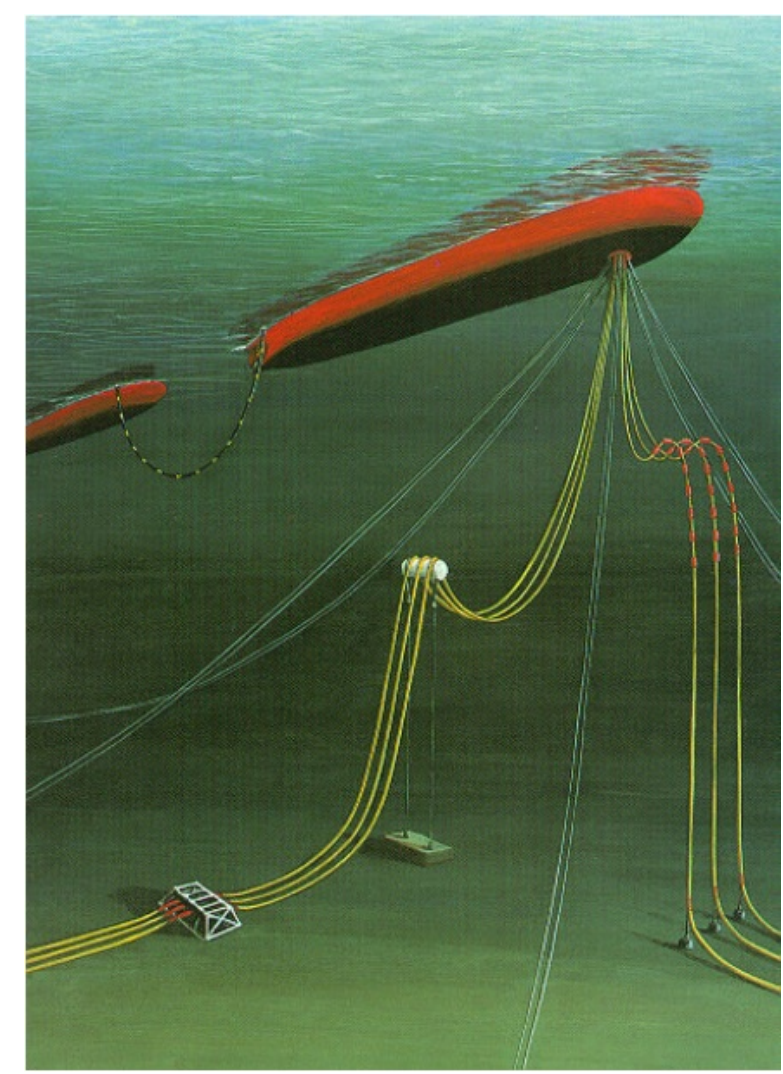

(b) SCR, lazy-wave and other riser configurations.

Source: Extracted from (a) - Kuiper (2008); (b) - Orcina Software. 
Risers are structures that transport, from the ocean floor till the ocean surface, economical commodities, e.g., oil and gas. Together with oil and gas, sea water, salt, sand, sediments, are also conveyed. Risers might be presented as a nearly rigid structure, for shallow waters, or flexible, for deep-water and ultra deep-waters fields. Besides, there are different configurations according to which a riser may be installed, such as, the Steel "Canetary" Riser (SCR), lazy-wave, top tensioned vertical riser, and so on; see Figure 1.1. What will determine the riser configuration is the sort of floating unit from which is suspended, the local depth and other in financial parameters.

As mentioned before, the riser dynamics is a subject in which several phenomena occur with different time and space scales. Among all sorts of Fluid-Structure phenomena, Vortex Induced Vibration (VIV) over the spanwise and parametric resonance due to heave motion imposed at the top of the line by gravitational waves over the vessel in which risers are suspended; are to be emphasized Figure 1.2. Generally, VIV and heave play concomitant action on the structure, however the present work will only deal with effects due to parametric resonance, modal structural parameters and hydrodynamical coefficients in still water.

Figure 1.2: Plataform and other ocean units on which risers might be used.

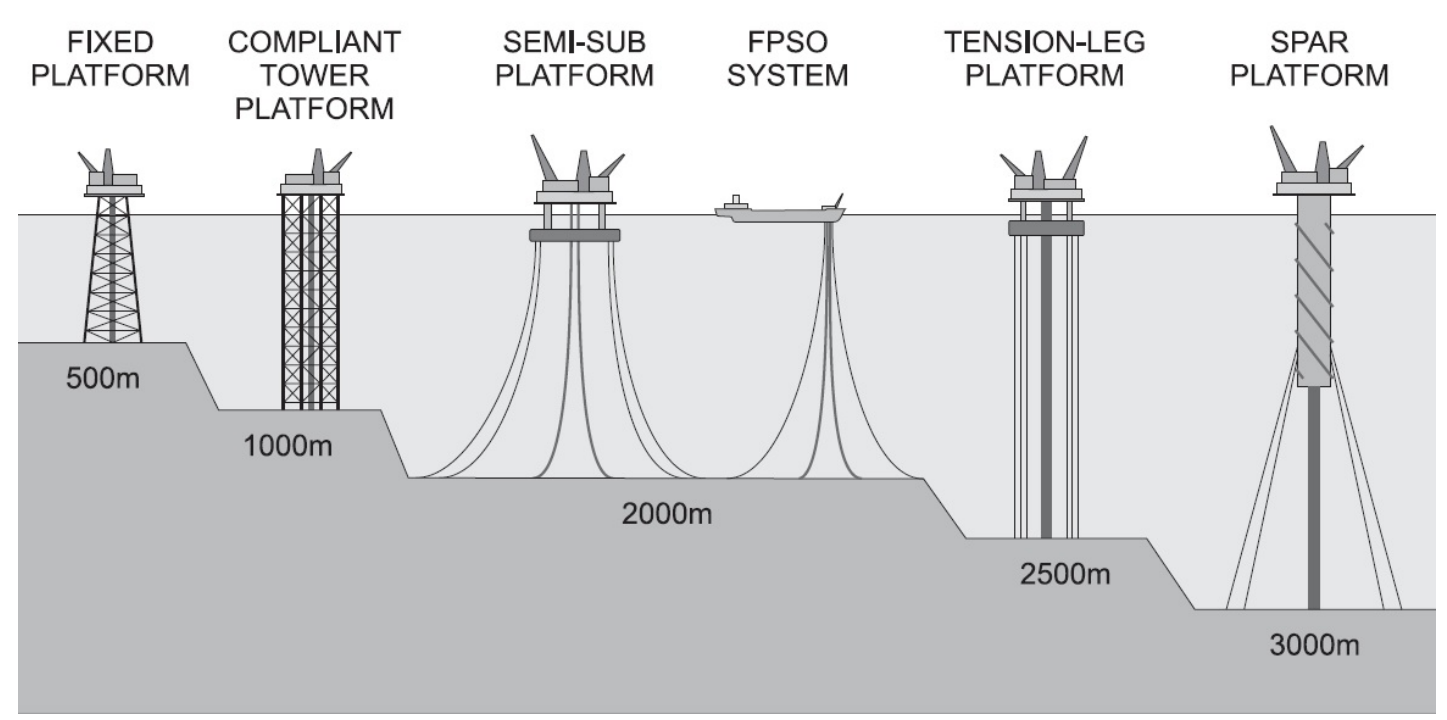

Source: Extracted from Assi (2009).

The small-scale model developed back in 2011 to conduct experimental campaigns at NDF, TPN and IPT will be soon detailed in Chapter 4. In order to study the elastica dynamical behavior in the experiments, it was proposed a non-intrusive observation using cameras capturing the Cartesian motion of an amount of optical targets attached in the model. The number of optical targets is 43 (experiments in water) and 54 (experiments in air).

Analyzing around 50 temporal series for each Cartesian direction of each target would be a huge work that not necessarily would show some correlations, or internal resonances easily. Following the Zeitgeist proposed in LIFE\&MO (2012d), the modal decomposition is studied and it is used to condensate the most part of the information contained in all temporal series of each target in a few modal ones.

The choice of a modal basis to conduct a modal decomposition is properly discussed 
in the light of Galerkin's method. The climax of modal decomposition is reached when a modal Mathieu-Hill oscillator is determined from a proposed theoretical continuum formulation of a heavy vertical line with imposed motion at the top in Chapter 3.

Considering initially the experimental results obtained in LIFE\&MO (2012d) from the perspective of the modal oscillator, it exists the possibility to define a few modal parameters, e.g., total modal mass, structural linear modal damping, modal structural stiffness and hydrodynamical non-linear damping. The total modal mass parameter is consisted of the structural mass (known) and the added mass (unknown) and the damping effect from both structural and hydrodynamical is amalgamated, being impossible to isolate one from the other without previously evaluating the structural linear damping.

The experimental campaign conducted in air comes to attention in order to evaluate the modal structural damping coefficient using a simple free-decay test. Besides, it is shown that it is also possible to estimate directly the modal added mass parameter when both experiments in water and in air are compared one to the other in a certain physical condition of modal structural stiffness equivalence between the experiments proposed.

Exploring the modal Mathieu-Hill oscillator, the experiment in water presents a non-linear term due to hydrodynamical damping mainly caused by drag forces, being necessary to use a perturbation techniques proposed by Ramani et al. (2004), yielding to a local solution in the instability area in Strutt diagram. Finally, it is possible to study the modal stability by means of Strutt diagram, looking for results that might be inside any instability tongue.

The text is organized as follows:

Chapter 1: This is the present Chapter, dealing with this text context and main points of organization;

Chapter 2: The Literature Review is dedicated to invite the reader to discuss about the fundamental bibliography of topics that will be presented in this work. The author aimed at giving some historical and philosophical context, with some contemplative and reflexive thoughts;

Chapter 3: The Theoretical Background chapter acts as door into the mathematical and physical realms, from which the fundamental theoretical basis to deal with the phenomenology that appears inhere arises;

Chapter 4: The presentation of the methodology used to conduct an experimental test of free-decay and imposed heave motion at the top, similar to the one described in (LIFE\&MO, 2012d) is given;

Chapter 5: The Analysis and Experimental Methodology chapter brings a discussion about the work carried out in the present work;

Chapter 6: The Results obtained using the methodology described in the previous chapters and a few suggestions of further works are addressed; 
Chapter 7: The References section presents an unique survey of works from which some useful insights, data, figures, thoughts, or any other form of acknowledgment has been extracted. 


\section{Chapter 2}

\section{Literature review}

The stilly murmur of the distant Sea

Tells us of silence.

And that simplest Lute,

Placed length-ways in the clasping casement,

[hark!

$[\ldots]$

O! the one Life within us and abroad,

Which meets all motion and becomes its soul,

A light in sound, a sound-like power in light,

Rhythm in all thought, and joyance everywhere-

Methinks, it should have been impossible

Not to love all things in a world so filled;

Where the breeze warbles, and the mute still air Is Music slumbering on her instrument.

"The Eolian Harp" (excerpt) by Samuel Taylor

Coleridge, 1795.

One might say the natural order of nature is to have no order at all. The apparent order humankind supposes to perceive is an illusion caused by the absence of two very conflicting panoramas, by being unable to regard the whole universe at once, or to see on quantum scale. However, it is possible to find some order on the way in which mankind interacts and this fact could be pointed out as a great sparkle that has puzzled many ingenious minds since the establishment of the human race.

Philosophy and Mathematics are not the same, although they have a non-empty intersection. Clearly both subjects are infinite on their very own way, having a lot in common, but mathematics has some impishness that the other does not. If it is possible to conceive the idea that all sciences have come from the marriage between philosophy and mathematics, mechanics would have been their first child. In this context, Mathematics shall be understood as a language to express natural phenomenon concepts which one may gaze through Philosophy, as far as art, music and literature are a sort of language with whom one desires to express what their soul longs to say. 
Historically, the first mathematical language well defined was geometry, as it may be regarded with fundamental works of Euclid (c. 300 BC, 2002) and many others mathematicians. Geometry is a powerful language that has been found on all mechanical works since the Archimedes' Principle of the Levers, one of the most fundamental principles of statics, as it is said by Lagrange (1736-1813):

Archimède, le seul parmi les anciens qui nous ait laissé quelque théorie sur
la mécanique dans ses deux livres de ÆEquiponderantibus, est l'auteur du
principe du levier, lequel consiste, comme tout le monde fait, en ce que si
un levier droit est chargé de deux poids quelconques placés de part et d'autre
du point d'appui à des distances de ce point réciproquement proportionnelles
aux mêmes poids, ce levier sera en équilibre et son appui sera chargé de la
somme des deux poids. (LAGRANGE, 1989, p. 2) ${ }^{1}$

As Dark Ages rose, science found a rich nest among Arabs, Chineses and Indians. The most significant contributions were led by Arabs who took into advantage all acknowledgment acquired by the Romans and placed in libraries across the Empire, e.g. Library of Alexandria. Among a few notable Arab mathematic findings, it is possible to mention: the definition of the zero number; algebraic notations which are used still nowadays; extensive studies with polynomials and their roots; numerical methods for evaluating square and cubic roots; and even introduction of the proof by induction.

The 17th century Enlightenment brought a breath of fresh air to science and, by the end of that century, mechanics would have been metamorphosed into a solid ground for those who wanted to wonder about the universe; the triad of works developed by Galilei (1638), Huygens (1673) \& Newton (1687) opened doors to a whole new world.

A truly important question had risen: what would the fundamental principle of mechanics be? Such an idea came from a principle Fermat (1601-1665) derived from Huygens-Fresnel principle in which light should always take a path between two points that can be transversed in the least time. Lanczos, in his masterpiece The Variational Principles of Mechanics, (Lanczos, 1970), wrote a brief introduction very clarifying about the philosophical evaluations of mechanics at the 17th-18th centuries.

\footnotetext{
${ }^{1}$ Archimedes, the only one among the ancients who has actually left us some theory about mechanics on his two books of AEquiponderantibus, is the author of the principle of the lever, which consists on the statement, as everyone does, that if a straight lever is to be loaded by any two weights placed on either side of its support point, placed at distances reciprocally proportional to those weights, the lever will be in equilibrium and its support point will be loaded with the sum of both weights. (Own translation)
} 
The keynote of that entire period was the seemingly per-established harmony between "reason" and "world". The great discoveries of higher mathematics and their immediate application to nature imbued the philosophers of those days with an unbounded confidence in the fundamentally intellectual structure of the world. [...] But this is not surprising if we observe how the purely æsthetic and logical interest in maximum-minimum problems gave one of the strongest impulses to the development of infinitesimal calculus, and how Fermat's derivation of the laws of geometrical optics on the basis of his "principle of quickest arrival" could not fail to impress the philosophically-orientally scientists of those days. (LANCZOS, 1970, p. xxvii)

The principle of least action appeared on mechanics, establishing a dual correspondence with Fermat's principle on geometrical optics ${ }^{2}$. This principle has many forms of being presented, but, for the sake of simplicity, it will be considered as Hamilton's principle. It is noteworthy that the principle of least action was misused by Maupertuis (1698-1759) and others on a metaphysical context; Lanczos, in (Lanczos, 1970, p. xxvii), also wrote about it arguing: "[...] the dilettante misuse of these tendencies [of world intellectual structure] [sic] by Maupertuis and others for theological purposes has put the entire trend into disrepute, is not the fault of the great philosophers". Lagrange, in his headstone Mécanique Analytique (Lagrange, 1989, p. 189), mentioned that "[...] est le Principe auquel je donne ici, quoique improprement le nom de moindre action, \& que je regarde non comme un principe métaphysique, mais comme un résultat simple \& général des loix [sic] de la Méchanique", consolidating the principle of least action only as a scientific principle on the analytical mechanics realm.

Although the classical holonomic Hamilton's principle is applicable for arbitrary systems, it is not quite general, because it only holds for systems characterized by monogenic forces and holonomic auxiliary conditions ${ }^{3}$. As a matter of fact, Hamilton's principle may be derived by the Principle of Virtual Work (PVW), already known in statics, together with d'Alembert (1717-1783) principle ${ }^{4}$. D'Alembert's principle is a bridge between dynamics and statics, bringing into light the possibility of finding a system of differential equations which describes the system dynamics, as much as the PVW shall be employed in order to find a system of algebraic equations which solves a static problem.

On the mechanics realm, it may be pointed out d'Alembert's principle and the PVW as fundamental principles of statics and dynamics. These two principles reproduce the Hamilton's principle and, by doing so, they reduce the fundamental problem of dynamics as a calculus of variations problem, a branch of calculus developed by Euler (1707-1783)

\footnotetext{
${ }^{2}$ In this case, the principle of least action shall be understood as Jacobi's principle.

${ }^{3}$ Conditions that can be written upon a differential form which may be integrated, also known as Pfaffian form.

${ }^{4}$ Later, by using a proper treatment on the Hamilton's principle, it has been generalized for systems with changing mass - e.g., Mclver (1973) - and for non-material systems; see Casetta and Pesce (2013).
} 
and Lagrange. This leads to Euler-Lagrange equations of movement, or even Hamilton equations; further acknowledgments may be obtained in Goldstein (1965), Lanczos (1970), Gantmacher (1975), Meirovitch (1980, 2003), Dugas (1988), Whittaker (1988), Lagrange (1989) \& Gelfand and Fomin (2000).

\subsection{Approaches to modeling and dynamical experimental analysis}

Inroads at the flexible cylinder system have been done over time using analytical, semi-analytical and numerical models. There are structural models from the simplest cable - under small displacement and linear-elastic behavior assumptions - in which the representation is done using bars and Euler-Bernoulli tensioned beams, to more complex models in which geometric non linearities or even non linear rheological behavior is taken into account.

Calling attention to the analytical and semi-analytical models, it is usual to consider a linear-elastic material. Asymptotic methods and perturbation techniques, e.g., WKB, allow to reach closed modal representations of the structure dynamics; see Pesce et al. (1999), Pesce and Martins (2005) \& Chatjigeorgiou (2008a, 2008b), all inspired on Triantafyllou (1984). The bending stiffness effect may also be taken into account analytically, by introducing an integral concept of equivalent geometric rigidity, as it might be seen in Mazzilli et al. (2014).

More sophisticated approaches, using non-linear modes, e.g., Shaw and Pierre (1991, 1993), have also reached interesting results. The counterpart of those approaches is the need for highly complex mathematical techniques, whereas they are based on the use of multiple scales methods or a proper finite-element formulation; see Sanches et al. (2007), Mazzilli (2009), LIFE\&MO (2011c, 2011d), Mazzilli and Sanches (2011), Mazzilli et al. (2011) \& Prado et al. (2013).

On the other hand, high-hierarchy numerical model approaches are based on a general Finite Element Method (FEM) formulation. This is an usual and classical approach; further acknowledgment might be seen on Pesce and Martins (2005), Huera-Huarte (2006), Pesce et al. (2006b), Silveira et al. (2007), Rustad et al. (2008), Morooka and Tsukada (2011), Takafuji and Martins (2012), Mainçon and Bernardo-Viljoen (2013) \& Prado et al. (2013). Numerical models that consider large rotations have shown to be a good approach for some stability investigations; see Gay Neto and Martins (2013).

Regarding the structure dynamical experimental analysis, several techniques have been employed. From classical techniques, e.g., Galerkin methods, achieving modal decomposition representations on a finite Hilbert space - e.g., Huera-Huarte (2006), Gu et al. (2013) \& Franzini et al. (2014) - to identification techniques of other natures, e.g., Kassen (2002), Lucor et al. (2006), Lucor and Triantafyllou (2008), Mukundan (2008), 
Mukundan et al. (2009) \& Mainçon and Bernardo-Viljoen (2013).

The modal decomposition has been widely used in recent publications in which experimental approaches are dealing with modal parameters as added mass and damping; see Franzini et al. (2015). There is also a branch using Reduced Order Models (ROMs) obtained from modal decomposition in order to study modal added mass and mean drag force affect; see Franzini et al. (2015) Franzini et al. (2016).

\subsection{Signal analysis techniques}

Inasmuch as the present work gets along with experimental data, it is also important to use proper signal analysis techniques which are more suitable for each modal or target time series. The experimental database obtained in LIFE\&MO (2012c, 2012d) shows a great deal of displacement signals, along with a load cell signal. As it may be seen in Franzini et al. (2014), such signals are extremely non-ergodic in some cases, presenting amplitude modulation and spectral effects from a different assortment of harmonics; in fact, Modarres-Sadeghi et al. (2011), via Poincaré mapping, have shown that there is a possible switch behavior on a same signal in which the structure might be periodical at one moment, being followed by a quasi-periodical or even chaotic behavior at another.

Spectral techniques as Fast Fourier Transform (FFT), Short Time Fourier Transform (STFT), Gabor Transform ${ }^{5}$ (GT) and Wavelet Transform (WT) might be used somehow to give a glimpse of the system dynamical behavior. A simple FFT can, at a first sight, tell a lot from the signal spectra, however the FFT analysis is best suitable for ergodic signals with no amplitude modulation; see Proakis and Manolakis (2007). On the other hand, the STFT and the GT would exhibit more reliable results, giving a Joint Time-Frequency Analysis (JTFA) with more details about the dynamics hidden on the temporal series; see Hamdan et al. (1996), Jubran et al. (1998) \& Ikoma et al. (2012). About JTFA using STFr and GT, Jubran et al. said:

\footnotetext{
${ }^{5}$ Gabor Transform is a special case of a Short Time Fourier Transform in which the window used is a Gaussian-type function.
} 
It is to be noted that STFT method is one of the most widely used classical methods of JTFA, which is also used, for the analysis of nonstationary signals where the time series is scanned in overlapping windows. Thus, STFT gives a redundant information and only a part of it is sufficient to synthesize exactly the input signal using the inverse STFT. Although, STFT suffers from many pitfalls, such as aliasing, time window effect, picket fence effects, and bandwidth-localization tradeoff. For example, for a large analysis window, low frequencies can be calculated, while high frequencies are poorly localized. On the other hand, the STFr in time and frequency domains can not be arbitrarily small because their product is lower banded [...]

Thus, for impulsive and nonstationary signals which requires good time frequency resolution, classical STFT ceases to be productive, since STFr can not either provide good frequency-resolution nor good time resolution. (JUBRAN ET AL., 1998, p. 585, emphasis added)

Thereby, a properly chosen wavelet base would be a better choice than a simpler STFr. As an alternative, there is the Hilbert-Huang Transform (HHT) which has been used on experimental data from fluid-structure interaction phenomena, specially VIV and VIM, since Pesce et al. (2006). The HHT has shown to be a very useful and reliable technique for the interpretation of phenomena in which amplitude-frequency modulation occurs; see Franzini et al. (2011a), Franzini et al. (2011b), Franzini et al. (2012), Gonçalves et al. (2012), Gonçalves et al. (2012), Franzini et al. (2013) \& Gonçalves et al. (2013). 


\title{
Chapter 3
}

\section{Theoretical background}

\begin{abstract}
"As a matter of fact, there is no mystery about these distortions of nature about which we still hear complaints in discussions on modern art. Everyone who has ever seen a Disney film or a comic strip knows all about it. [...] Mickey Mouse does not look very much like a real mouse, yet people do not write indignant letters to the papers about the length of his tail."

The Story of Art (excerpt) by Sir Ernst H. Gombrich.
\end{abstract}

Following, it will be presented the theoretical background of this present work, but, firstly, it shall be remarked how this chapter is organized. There are four main subjects to be placed on the theoretical background which refer to:

1. Development of small-scale model for the flexible vertical cylinder;

2. Experimental mathematical formulation:

(a) Approaches from different models;

(b) Eigenvalues and eigenfunctions: the Sturm-Liouville problem associated to each sort of model;

(c) Defining the suitable modal decomposition;

3. Modal oscillator

\subsection{Modal mathematical formulation}

\subsubsection{The classical vibrating string}

The vibrating string is a mechanical system in which there is a material medium, the string, that is able to transfer information from one point to another through a wave. That system is essentially one-dimensional, but it can be extended to higher dimensional applications 
on the theory of vibrating plates or bodies. The information is propagated along the vibrating string at the sound velocity, which is a directed function of the material medium (string) mechanical properties; see Rayleigh (1896). The called classical vibrating string presents axial constant tension at each point of the string.

A phenomena such as the string vibration and its effects over nature should be regarded as a puzzle that has been bewitching philosophers since Pythagoras (c. 570BC--c. 495BC). Historically, Pythagoras was the first philosophically oriented mathematician to demonstrate a proportional relationship between sound and the wavelength of a stationary vibrating string. Such proportional relationship is known as harmonic series; e.g., the harmonic series of $C_{2}$, see Figure 3.1, also known as $C$ on Helmholtz designation, Helmholtz (1885).

Figure 3.1: Harmonic series of $\mathrm{C}_{2}$

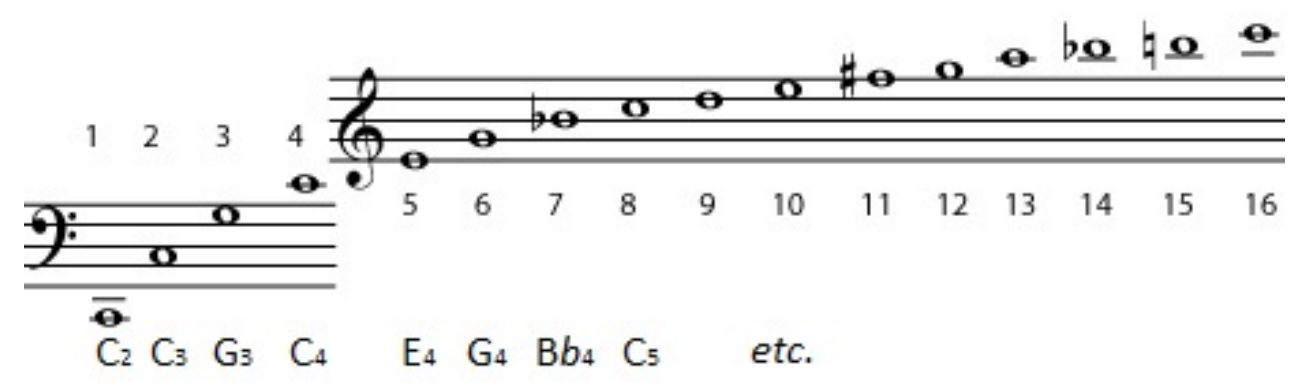

The idea behind the harmonic series is ingenious: Pythagoras stated that, given an ideal string with a fundamental frequency, every rational multiple of this fundamental frequency is related to a wavelength inversely proportional to the integer chosen; see Figure 3.2. From the harmonic series, it is possible to determine the perfect fifths ${ }^{1}$ chain (F-C-G-D-A-E-B) and, then, it is also possible to find the seven natural pitches of the diatonic scale, that are still used nowadays in music. Besides the chain of perfect fifths, the diatonic scale and harmonic series, Pythagoras' works on the vibrating string precede the Major scale, the lonian mode, a basis for musical composition.

\footnotetext{
${ }^{1} \mathrm{~A}$ perfect fifth is related to the third harmonic of the harmonic series proposed by Pythagoras; actually, the third harmonic is the perfect fifth of the second harmonic, so there is a ratio $2: 3$ between a pitch and its perfect fifth. For example, if $\mathrm{C}_{3}(130.81 \mathrm{~Hz})$ is considered to be the fundamental note, its perfect fifth would be the $\mathrm{G}_{3}(196.15 \mathrm{~Hz})$; see Figure 3.1 .
} 
Figure 3.2: Pythagorean relationship between frequency and wavelength in a ideal vibranting string

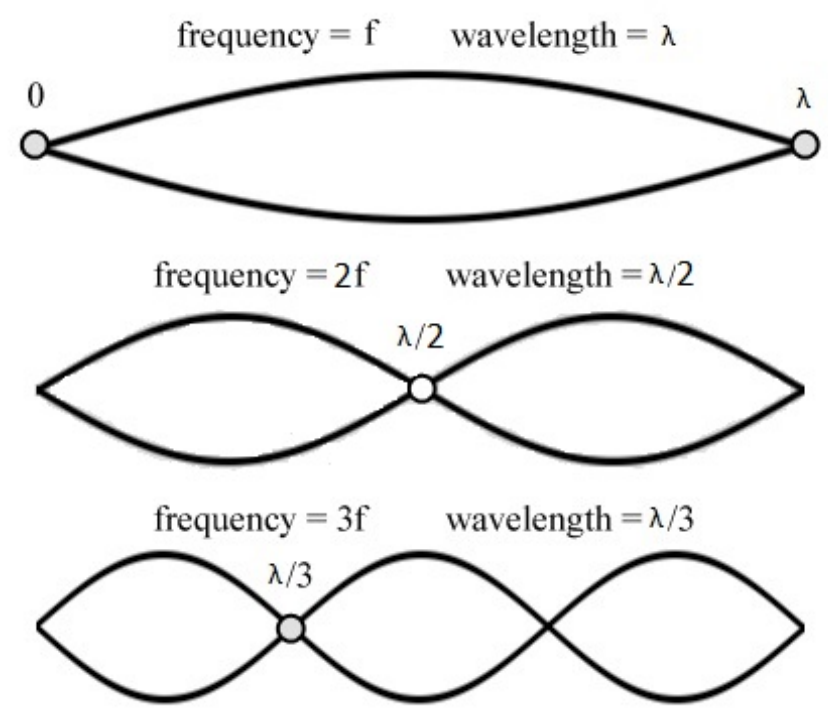

After this brief introduction, it will be presented a mathematical model to the classical vibrating string. The schematic model is seen in Figure 3.3.

Figure 3.3: Schematic model of a classical vibrating string bi-articulated with constant distributed mass, $\mu$, and constant traction, $T$.
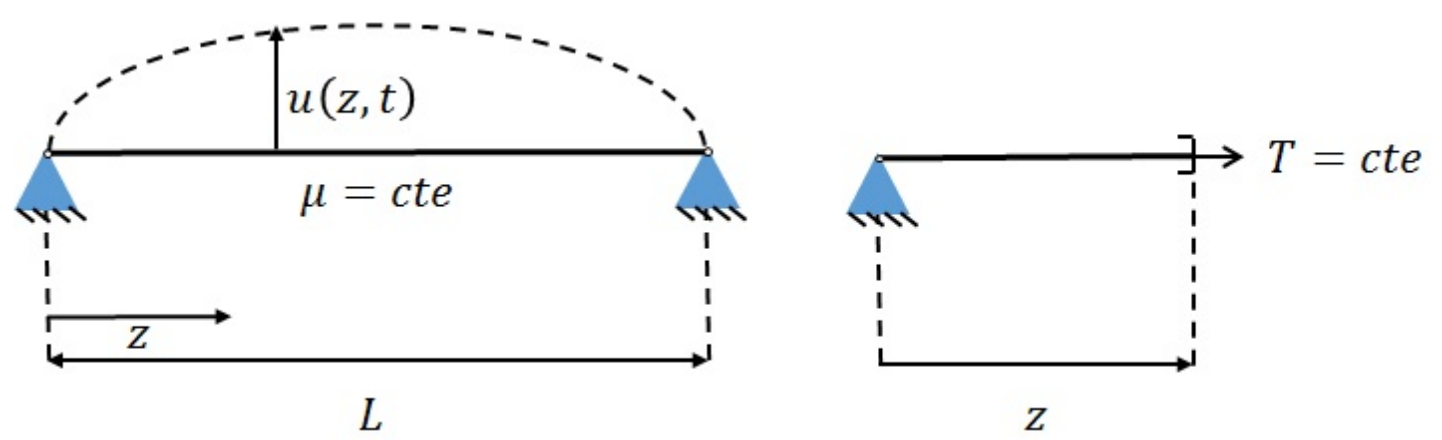

The string is supposed to have constant length, even when it is transversally deformed and it is considered bi-articulated in order to neglect flexural effects near its ends. The transversal displacement is given by $u(z, t)$. The stationary wave equation, eq. 3.1, will be used as a mathematical model.

$$
\mu(z) \frac{\partial^{2} u}{\partial t^{2}}=\frac{\partial}{\partial z}\left[T(z) \frac{\partial u}{\partial z}\right]
$$

For constant tension, it is possible to rewrite eq. 3.1 as follows:

$$
\frac{\partial^{2} u}{\partial t^{2}}=\frac{T}{\mu} \frac{\partial^{2} u}{\partial z^{2}}=c_{0}^{2} \frac{\partial^{2} u}{\partial z^{2}}
$$


In which $c_{0}=T / \mu$ is the wave celerity. The variable separation method is employed on $u(z, t)=\psi(z) \varphi(t)$ in order to uncouple both variables; see Tikhonov and Samarskii (1980). Using the variable separation proposed before on eq. 3.2, it is possible to write ${ }^{2}$ :

$$
\psi(z) \ddot{\varphi}(t)=c_{0}^{2} \psi^{\prime \prime}(z) \varphi(t) \Leftrightarrow \frac{\ddot{\varphi}(t)}{\varphi(t)}=c_{0}^{2} \frac{\psi^{\prime \prime}(z)}{\psi(z)}=-\Omega^{2}=c t e
$$

After some algebraic manipulation, regarding $\varphi(t)=A \exp [j(\Omega t+\theta)]$ with $j^{2}=-1$, $\psi(z)=B \cos (k z)+C \sin (k z)$ and boundary conditions $u(0, t)=u(L, t)=0$, leading to $B=0$, the solution 3.3 takes form of a infinite series given by:

$$
u(z, t)=\sum_{n=1}^{\infty} D_{n} \Re\left[e^{j\left(\Omega_{n} t+\theta_{n}\right)}\right] \sin \left(i \frac{\pi}{L} z\right), \text { where } \Omega_{n}=n \frac{\pi}{L} c_{0}=n \Omega, n=1,2, \ldots
$$

The Pythagorean harmonic series aforementioned is given in eq. 3.4 as a natural result, $\Omega_{n}=n \Omega$. In addition, it is also evaluated the eigenfunction for each modal shape, $\psi_{n}(z)=\sin (n \pi z / L)$. In practical methods, the infinite sum presented on eq. 3.4 will become finite, being truncated some high-harmonic, $N \in \mathbb{N}$; see eq. 3.5.

$$
u(z, t)=\sum_{n=1}^{N} D_{n} \Re\left[e^{j\left(\Omega_{n} t+\theta_{n}\right)}\right] \sin \left(n \frac{\pi}{L} z\right), \text { where } \Omega_{n}=n \frac{\pi}{L} c_{0}=n \Omega, i=n, 2, \ldots, N
$$

The equation 3.2 is a 2 nd order equation in $t$ and $z$, which means it would be necessary 4 informations in order to evaluate all constants $-D_{n}, \theta_{n}, A_{n}$ and $C_{n}$ - and find the dynamical response of the system. Two informations were given as boundary conditions, which determine the coefficients $A_{n}$ and $C_{n}$. Initial conditions are missing in order to determine the other two coefficients. For the present work, only the Sturm-Liouville problem is necessary; therefore, the modal shapes (eigenfunctions), $\psi_{n}$, and natural frequencies (eigenvalues), $\Omega_{n}$, evaluated using the boundary conditions are desirable.

\subsubsection{Vertical string}

Next, it will be introduced the vertical string, a vibrating string in which the effect of its own weight causes a variable traction along its span; see Figure 3.4. Some of the assumptions made for the classical vibrating string still holds, as: constant length (even when deformed), flexural effects on both endings are neglected and stationary waves.

\footnotetext{
${ }^{2}$ In this text, $\dot{\square}$ refers to temporal derivatives; and $\square$ ', to space derivatives.
} 
Figure 3.4: Vertical tensioned string subjected to its own weight

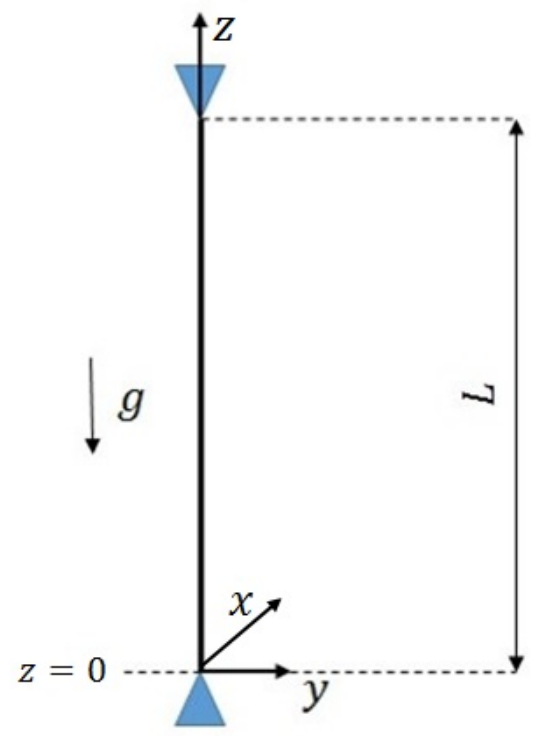

Similarly to that was done before, the main idea is to start with the wave equation, see eq. 3.1, in order to determine the eigenvalues and eigenfunctions of the vertical vibrating string. Again it will be supposed a transversal displacement along the string, $u(z, t)$, which can be decomposed into the Cartesian directions presented in Figure 3.4, as $\vec{u}(z, t)=u^{x}(z, t) \vec{\imath}+u^{y}(z, t) \vec{\jmath}$. The traction on the vertical string shall be presented as a function of the position of the string, $T(z)$, due to the effect of its own weight; see eq. 3.6.

$$
T(z)=T_{0}+\gamma z=T_{0}+\mu g z
$$

Firstly, it is necessary to rewrite the wave equation, considering a non-constant traction and, after that, the separation variable method shall be employed as well, $u(z, t)=\psi(z) \varphi(t)$; see eqs. 3.7-3.8 and Tikhonov and Samarskii (1980).

$$
\begin{gathered}
\mu \frac{\partial^{2} u}{\partial t^{2}}=T^{\prime}(z) \frac{\partial u}{\partial z}+T(z) \frac{\partial^{2} u}{\partial z^{2}} \\
\frac{\ddot{\varphi}(t)}{\varphi(t)}=\frac{T^{\prime}(z) \psi^{\prime}(z)+T(z) \psi^{\prime \prime}(z)}{\mu \psi(z)}=-\Omega^{2}
\end{gathered}
$$

After some trivial algebraic manipulation, it is possible to find eq. 3.9 which is a modified Bessel equation ${ }^{3}$. In order to reach the traditional Bessel equation, it will be necessary to introduce two change of variables, as follows; see 3.8.

\footnotetext{
${ }^{3}$ As said before, the main purpose is to reach the eigenvalues and the eigenfunction of the
} 


$$
\begin{gathered}
\left(\frac{T_{0}}{\gamma}+z\right) \psi^{\prime \prime}(z)+\psi^{\prime}(z)+\frac{\mu \Omega^{2}}{\gamma} \psi(z)=0 \\
\tilde{\zeta}^{2}(z)=\frac{T_{0}}{\gamma}+z \\
\tilde{\xi}(z)=\tilde{\zeta}=\sqrt{\frac{T_{0}}{\gamma}+z}
\end{gathered}
$$

The following algebraic steps will be omitted due to a tedious process of differentiations and substitutions that will result in eq. 3.11, a Bessel equation whose solution is given by eq. 3.12; further discussion in Bowman (1958) and Pesce et al. (1999).

$$
\begin{gathered}
\tilde{\xi}^{2} \frac{d^{2} \psi}{d \tilde{\xi}^{2}}+\frac{d \psi}{d \tilde{\xi}}+4 \tilde{\xi} \tilde{\beta}^{2} \tilde{\xi}^{2} \psi=0, \text { where } \tilde{\beta}^{2}=\Omega^{2} \frac{\mu}{\gamma} \\
\psi(z)=C_{1} J_{0}(2 \tilde{\beta} \tilde{\xi}(z))+C_{2} Y_{0}(2 \tilde{\beta} \tilde{\xi}(z))
\end{gathered}
$$

Where $J_{0}$ and $Y_{0}$ are Bessel functions of first and second kind of zeroth-order, respectively. The only requirement for the solution to exist ${ }^{4}$ until now is $\tilde{\xi}(0)>0$, inasmuch as $Y_{0}\left(z \rightarrow 0^{+}\right) \rightarrow-\infty$. The next step is to use the boundary conditions, $\psi(0)=\psi(L)=0$, in order to find the unknowns coefficients $C_{1}$ and $C_{2}$. It is possible to write the boundary conditions in matrix form, see eq. 3.13, and, ignoring the trivial answer, it will be determined a sufficient condition given by eqs. 3.14-3.15.

$$
\left[\begin{array}{ll}
J_{0}(2 \tilde{\beta} \tilde{\xi}(0)) & Y_{0}(2 \tilde{\beta} \tilde{\xi}(0)) \\
J_{0}(2 \tilde{\beta} \tilde{\xi}(L)) & Y_{0}(2 \tilde{\beta} \tilde{\xi}(L))
\end{array}\right]\left\{\begin{array}{l}
C_{1} \\
C_{2}
\end{array}\right\}=\left\{\begin{array}{l}
0 \\
0
\end{array}\right\}
$$

Sturm-Liouville problem associated to the vertical string model. Thereby, it will be considered only the function $\psi(z)$ on the analysis. For further discussion about the Sturm-Liouville problem, see Hönig (1978).

${ }^{4}$ The requirement that $\xi(0)>0$ means that the traction at the bottom must never be null, $T_{0}>0$. Such deficiency of the model comes from the neglected flexural rigidity near the ends. 


$$
\begin{gathered}
\left|\begin{array}{ll}
J_{0}(2 \tilde{\beta} \tilde{\xi}(0)) & Y_{0}(2 \tilde{\beta} \tilde{\xi}(0)) \\
J_{0}(2 \tilde{\beta} \tilde{\xi}(L)) & Y_{0}(2 \tilde{\beta} \tilde{\xi}(L))
\end{array}\right|=0 \Leftrightarrow \\
\Leftrightarrow f\left(\tilde{\beta}_{n}\right)=J_{0}\left(2 \tilde{\beta}_{n} \tilde{\xi}(0)\right) Y_{0}\left(2 \tilde{\beta}_{n} \tilde{\xi}(L)\right)-Y_{0}\left(2 \tilde{\beta}_{n} \tilde{\xi}(0)\right) J_{0}\left(2 \tilde{\beta}_{n} \tilde{\xi}(L)\right)=0 \\
C_{2 n}=-\frac{J_{0}\left(2 \tilde{\beta}_{n} \tilde{\xi}(0)\right)}{Y_{0}\left(2 \tilde{\beta}_{n} \tilde{\xi}(0)\right)} C_{1 n}=-\frac{J_{0}\left(2 \tilde{\beta}_{n} \tilde{\xi}(L)\right)}{Y_{0}\left(2 \tilde{\beta}_{n} \tilde{\xi}(L)\right)} C_{1 n}
\end{gathered}
$$

As it can be seen, equation 3.14 is a transcendental equation that presents an infinite number of zeros, $\tilde{\beta}_{n} \in \mathbb{R}$ with $n \in \mathbb{N}$, such that the relation $f\left(\tilde{\beta}_{n}\right)=0$ is satisfied. In order to find any $\tilde{\beta}_{n}$, it is necessary to use a graphical or a numerical method. It is noteworthy that the parameter $\tilde{\beta}_{n}$ is related to the eigenvalues, $\Omega_{n}$, of the problem; see eq. 3.11. The closed-form solution for the eigenfunction relative to the $n$-th mode is given by eq. 3.16.

$$
\begin{aligned}
& \psi_{n}(z)=C_{1 n}\left[J _ { 0 } \left(2 \Omega_{n} \sqrt{\frac{\mu}{\gamma}} \sqrt{\left.\frac{T_{0}}{\gamma}+z\right)}-\frac{J_{0}\left(2 \tilde{\beta}_{n} \sqrt{\mu T_{0}} / \gamma\right)}{Y_{0}\left(2 \tilde{\beta}_{n} \sqrt{\mu T_{0}} / \gamma\right.} \times\right.\right. \\
&\left.\times Y_{0}\left(2 \Omega_{n} \sqrt{\frac{\mu}{\gamma}} \sqrt{\frac{T_{0}}{\gamma}+z}\right)\right], \text { where } z \in[0, L] \subset \mathbb{R}
\end{aligned}
$$

Considering, without loss of generalization, $C_{1 n}=1$, and including a new variable, $\hat{z}=z / L$, it is possible to reach a closed-form solution of the eigenfunction in respect to the normalized string length, $\hat{z} \in I=[0,1] \subset \mathbb{R}$; see eq. 3.17. Finally, a normalized eigenfunction, see eq. 3.18, might be presented to the vertical heavy string problem in which the tension on the bottom end is always positive (under traction), $T_{0}>0$. The normalized mode is useful in order to perform qualitative comparisons between the unitary trigonometric (sine) eigenfunctions, see eq. 3.5, and the present eigenfunctions (Bessel modes); see Figure 3.5a-d.

$$
\begin{aligned}
\psi_{n}(\hat{z})=\left[J_{0}\left(2 \Omega_{n} \sqrt{\frac{\mu L}{\gamma}} \sqrt{\frac{T_{0}}{\gamma L}+\hat{z}}\right)-\right. & \left.\frac{J_{0}\left(2 \Omega_{n} \sqrt{\mu T_{0}} / \gamma\right)}{Y_{0}\left(2 \Omega_{n} \sqrt{\mu T_{0}} / \gamma\right.}\right) \\
& \left.\times Y_{0}\left(2 \Omega_{n} \sqrt{\frac{\mu L}{\gamma}} \sqrt{\frac{T_{0}}{\gamma L}+\hat{z}}\right)\right], \text { where } \hat{z} \in I
\end{aligned}
$$




$$
\hat{\psi}_{n}(\hat{z})=\frac{\psi_{n}(\hat{z})}{\max \left\{\left\|\psi_{n}(\hat{z})\right\|\right\}}, \forall(n, \hat{z}) \in \mathbb{N} \times I
$$

Figure 3.5: Sine and Bessel modes: 1st-to-4th mode. Note: $\mu=1.58 \mathrm{~kg} / \mathrm{m}, L=2.6 \mathrm{~m}, \gamma=11.74 \mathrm{~N} / \mathrm{m}$, $T_{0}=9.44 \mathrm{~N}, \Omega_{n}=n 0.725 \mathrm{~Hz}$.

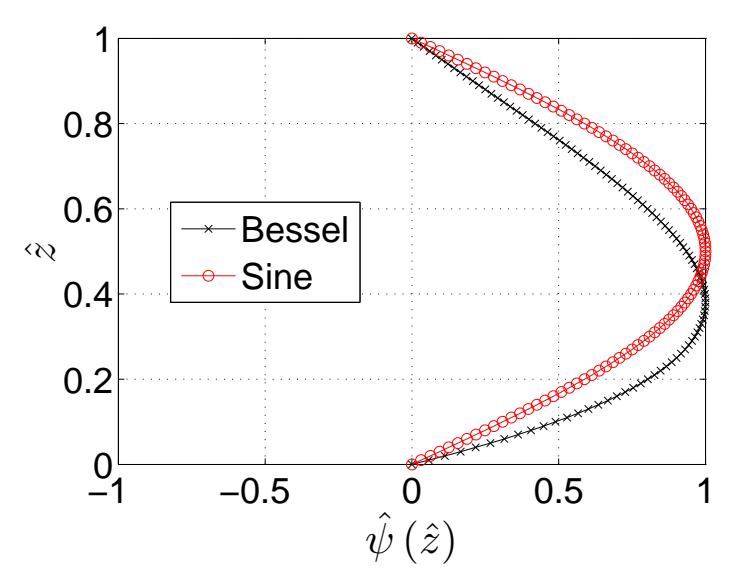

(a) First mode.

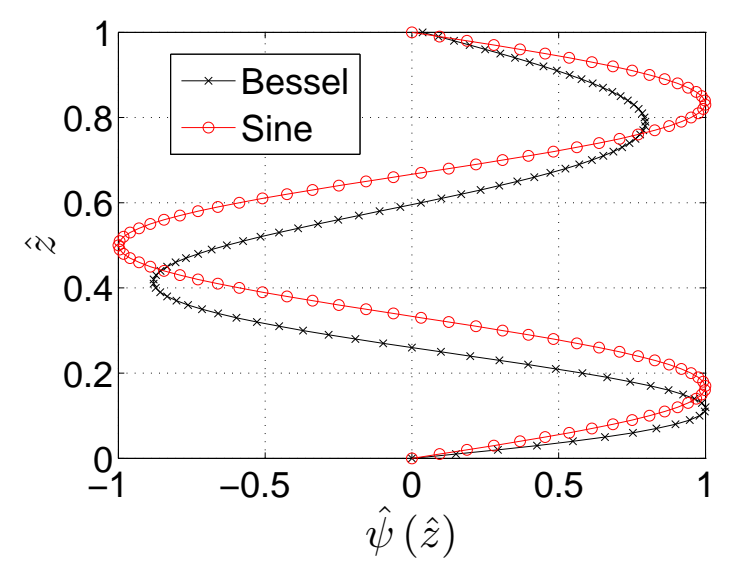

(c) Third mode.

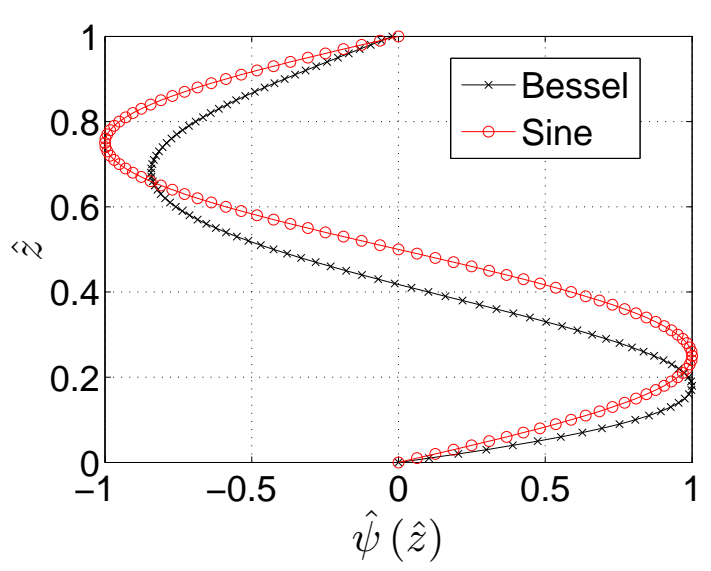

(b) Second mode.

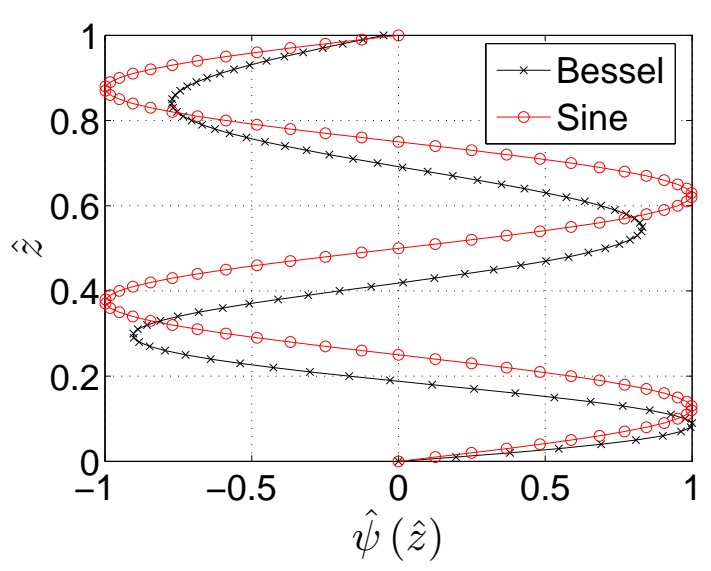

(d) Forth mode.

A similar system, the heavy hung chain simply supported at the top, is presented on Bowman (1958) and it has a similar solution to the vertical heavy string. Another very interesting approach was proposed by Mazzilli et al. (2014) in which the flexural rigidity is considered along the cylinder span as a "distributed load" and this load is approximated by an equivalent normal force proposed in their work. Doing such a trick, the fourth-order beam equation becomes a second-order one, resembling the Bessel formulation. Mazzilli et al. (2014) approach calls the eigenfunctions obtained as quasi-Bessel modes, from which they developed asymptotic expansions, reaching a closed analytical form for those. 


\subsubsection{Modal decomposition}

Modal decomposition is a powerful tool developed in late nineteenth century and early twentieth century, with contributions from Rayleigh (1896), Ritz (1909) \& Galerkin (1915). Modal analysis is intimately related to musical acoustics. As it might be seen in publications of middle-late nineteenth century, musical related studies, e.g., sound phenomenology, instruments and concert hall acoustics, were very common, since science also reflects the Zeitgeist.

At that time, analytical mechanics and the Hamilton theory of quaternions, see Hamilton (1853), were well-established among scientists. Ritz (1909) showed that the modal shape was directly linked to the minimization of a functional, being possible to analytically determine the mode of a mechanical system. The Rayleigh-Ritz method ${ }^{5}$ and the Galerkin-Ritz, see Galerkin (1915), are attempts to analyze a continuum problem from a discrete mesh, reducing the order of the system. Both methods are numerical and iterative, based on the search for three parameters: eigenvalues (frequencies), eigenfunction (modal shapes) and damping coefficients.

In order to find any of those parameters, it is necessary to guess how the modal shape would be like. The only restriction for a function to be candidate to represent a modal shape is to satisfy all boundary constraint. The quality of the method is defined by how better was the modal shape function (educated) estimation.

Computational methods, e.g., FEM, are based on Rayleigh-Ritz-Galerkin method inasmuch as they use a discrete mesh to discretize the continuum. The quality of a FEM analysis is related to the number of mesh elements, because they try to use as simple as possible polynomial functions to interpolate the continuum behavior: with a more refined mesh, the result will be better. There is a computational cost discussion that should be regarded, but it is not the main subject of the present work.

Experimental analysis commonly uses modal techniques, trying to reduce the order of a continuum system into a finite number of dof discrete system. Ewins (2000) is a masterpiece for experimental modal analysis, showing the fundamental points to perform a good experimental test and a posterior analysis.

The experimental data collected in LIFE\&MO (2012d) used a model in which discrete optical targets were fixed. The optical tracking system, Qualys ${ }^{\circledR}$, recorded displacement data from 43 discrete points of the tested model elastica. As it has been said, the phenomenon observed is complex, with many nuances and different response types, so that it is nearly impossible to extract much information when it is necessary to analyze 43 temporal series in parallel. An interesting manner to filter the data, minimizing the loss

\footnotetext{
${ }^{5}$ In a recent article, Leissa (2005) discuss that, in fact, the Rayleigh-Ritz method is strictly Ritz method. The Ritz method is similar to Rayleigh method - both minimize an energy functional - but they are different one from another. The aforementioned article has promoted a discussion on the same Journal years later, see llanko (2009), with some other considerations about the historical analysis of Rayleigh-Ritz method.
} 
of information, is changing the analysis point of view from the physical model to a modal model.

The continuum model has infinite modes, it has infinite dof. As much as the tested model is continuum, it was necessary to fix discrete targets on it, then what really exists is a finite number of dof from which it is possible to interpolate an elastica at each time instant. Therefore, with the data obtained, it would be possible to study the same finite number of modes. Would it be necessary to regard all 43 modes?

The answer is clearly negative, because it is possible to create a criteria of choosing only the most significant modes. A significant mode would present non-negligible amplitudes (or energy), which can be verified by a spectral analysis, such as FFT; see Figure 3.6. Another way to check if a certain amount of modes is sufficient to represent the whole model dynamics is comparing the elastica from the modal reconstitution with the experimental at each time increment.

Figure 3.6: Free-decay test in water on the experimental model. Power spectrum of the transversal movement amplitude along the model spanwise.

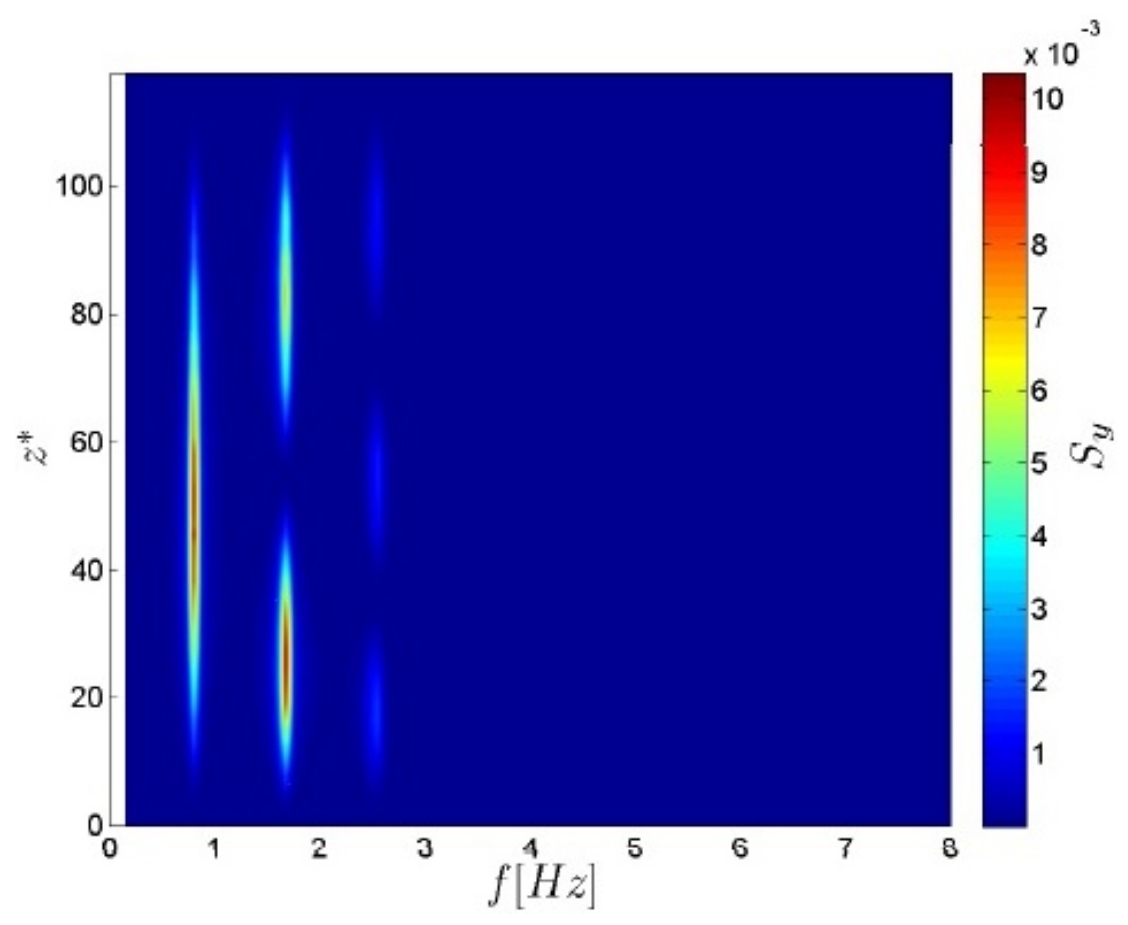

Source: Extracted from LIFE\&MO (2012d).

Figure 3.6 shows that the structure keeps the main energy introduced in it the first three modes. It would be needed a similar analysis to every experimental test, with VIV, imposed heave, concomitant VIV and heave, to conclude if only those modes are sufficient to represent the model dynamics. However an educated estimation would be that it is not many more modes than those, perhaps some mode should be included, but the first three should probably not be excluded. Another interesting thing can be seen 
from Figure 3.6 is that the experimental mode is very similar to sine and Bessel modes discussed earlier. In special, it resembles the Bessel mode asymmetry.

Regarding the continuum hypothesis, any solution, $u(z, t)$, for the vertical flexible cylinder elastica shall also be continuous and have continuous derivatives. It will be required that the solution is in a subset of Hilbert space, $\Lambda \subset H^{2}(\mathbb{R})$. There is a norm defined on $\Lambda$, such as:

$$
\|u\|=\langle u, u\rangle^{1 / 2}, \forall u \in \Lambda
$$

In which $\langle\cdot, \cdot\rangle$ represents an inner product still to be defined. Using the separation of variables method, it is considered that any vector, $u(z, t) \in \Lambda$, can be decomposed as:

$$
\underset{\sim}{u}(z, t)=\underset{\sim}{\psi}(z) \varphi(t)=A(t) \underset{\sim}{\psi}(z)
$$

In which $\varphi(t)=A(t)$ is the amplitude temporal series. Thereby, the modal decomposition is defined as a projection of the elastica displacement, $u(z, t)$, at each instant of time to a finite base formed with chosen modal shapes, $\{\Psi(z)\}$; see eqs. 3.21-3.24.

$$
\begin{gathered}
\{\underset{\sim}{\Psi}(z)\}=\left\{\underset{\sim}{\left.\psi_{1}(z), \underset{\sim}{\psi_{2}}(z), \ldots, \underset{\sim}{\psi_{N}}(z)\right\}}\right. \\
\underset{\sim}{u}(z, t)=\sum_{n=1}^{N} A^{n}(t) \underset{\sim}{\psi_{n}}(z)=A^{n}(t) \underset{\sim}{\psi_{n}(z)} \\
A^{n}(t)=\operatorname{proj}_{\sim} u(z, t)=\frac{\left\langle u(z, t), \sim_{\sim}^{\psi_{n}}(z)\right\rangle}{\left\langle{\left.\underset{\sim}{\psi_{n}}(z),{\underset{\sim}{\psi}}_{n}(z)\right\rangle}^{\langle u}\right.} \\
\langle u, v\rangle=\int_{0}^{L} w(z) u(z) v(z) d z, \forall(u, v) \in \Lambda \times \Lambda
\end{gathered}
$$

The inner product ${ }^{6}$ is the typical function space inner product with a weighting function, $w(z)$. The weighting function is considered to show some versatility of the

\footnotetext{
${ }^{6}$ If the normalized length, $\hat{z}$, is used, the inner product will be rewritten considering the Jacobian of the variable transformation, $\|J(z, \hat{z})\|$ :
} 
method, although it is usual to choose a unitary weight. In the case of Bessel functions, the natural weighting function would be $w(z)=z$, as it can be seen in Bowman (1958) \& Abramowitz and Stegun (1972). A suitable choice of an inner product can simplify analytical expressions. However, in the modal decomposition case, all calculations will be performed numerically, so it is not necessary to choose a natural weighting function.

The last, but not the least, point to be discussed is about the Galerkin method and the experimental tests with imposed heave motion. As it was mentioned before, the sufficient condition on Galerkin decomposition is to use a function which does not violate the systems constraint, a condition that resembles the analytical mechanics concept of virtual displacement, e.g., $\delta u$.

The modal representation base, $\{\Psi(z)\}$, see eq. 3.21, is chosen to be only a vector of the spanwise curved; being a fixed base for every time instant, it is time-invariant. Accordingly, it has emerged a dichotomy: is it possible to use such modal base to analyze experimental tests in which occur heave motion?

Heave motion simulates the effects of floating units in which a real riser might be fixed and those effects are crucial on riser dynamics, inasmuch as they represent external forces that may act on internal and parametric resonances. Trigonometric and Bessel-like modes use a fixed model length, $L$, in their closed-form solution. On the other hand, there is an elongation in heave tests in which the length becomes a function of time. Therefore, it would be possible to obtain a time-variant modal base.

In order to project in a time-invariant modal base, it is necessary to choose a length mean value. Galerkin's method is still valid, as the longitudinal displacement varies, the transversal does not violates the system constrains. Mazzilli et al. (2014) presents a closed-form solution for modes in which the elongation is considered and it is expected that the quality of a quasi-Bessel projection for a heave test should be better than a trigonometric ou Bessel-like.

In fact, the Rayleigh quotient might help to justify the use of the time-invariant base on the imposed heave tests. By choosing any close-to-the-mode function, the iterative method will present an error of second-order on the eigenvalue. Therefore, the error caused on the analysis using the time-invariant base with $1 \%$ of model length heave motion should not have any significant effect on the temporal modal amplitude series obtained.

$$
\langle u, v\rangle=\int_{0}^{L} w(z) u(z) v(z) d z=\int_{0}^{1} w(\hat{z}) u(\hat{z}) v(\hat{z})\|J(z, \hat{z})\| d \hat{z}=L \int_{0}^{1} w(\hat{z}) u(\hat{z}) v(\hat{z}) d \hat{z}
$$




\subsection{Modal oscillator}

The following section is devoted to propose a modal oscillator, based on a continuum model, $T(z, t)$. On the other hand, the heave will be considered as an axial tension modulation of the model. A damping term proportional to the velocity, $c_{s} \underset{\sim}{\dot{u}}$, is considered as an approximation of a viscous structural damping and a damping term proportional quadratically in the velocity (hydrodynamical drag force-Morisson's drag) is also present on the continuum model.

\subsubsection{Mathematical formulation from the continuum}

The continuum mathematical formulation is made from a wave equation with damping terms, considering the added mass effect; see eq. 3.25, Tikhonov and Samarskii (1980).

$$
\left(\mu+m_{a}\right) \frac{\partial^{2} \underline{u}}{\partial t^{2}}+c_{s} \frac{\partial \underline{u}}{\partial t}+\frac{1}{2} \rho_{w} D C_{D} \frac{\partial \underline{u}}{\partial t}\left\|\frac{\partial \widetilde{u}}{\partial t}\right\|=\frac{\partial}{\partial z}\left(T(z, t) \frac{\partial \underline{u}}{\partial z}\right)
$$

The tension term is defined in eq. 3.26 in which $T_{t}=T(z=L)$ is the tension at the experimental top, $A_{t}$; the heave motion amplitude; and $\omega_{t}$, the imposed movement frequency (in $\mathrm{rad} / \mathrm{s}$ ). As presented in Table 4.3, $E A$ is the axial stiffness of the experimental model (as built), the traction modulation is evaluated based on a simply rod model in which $E A / L$ would represent an equivalent spring constant.

$$
T(z, t)=T_{t}-\gamma(L-z)+T_{d}, \text { with } T_{d}=\frac{E A}{L} A_{t} \cos \left(\omega_{t} t\right)
$$

It is noticeable that there is an equivalent on writing the tension term using $T_{0}$ or $T_{t}$, see eqs. $3.6 \& 3.26$, respectively: $T_{t}-\gamma(L-z)=T_{0}+\gamma z$. Calling again the separation of variables method, the modal representation of the elastica, $u(z, t)=$ $\varphi^{n}(t){\underset{\sim}{n}}_{n}(z)$, with $n=1,2, \ldots, N$, and rewriting eq. 3.25 in a more convenient manner:

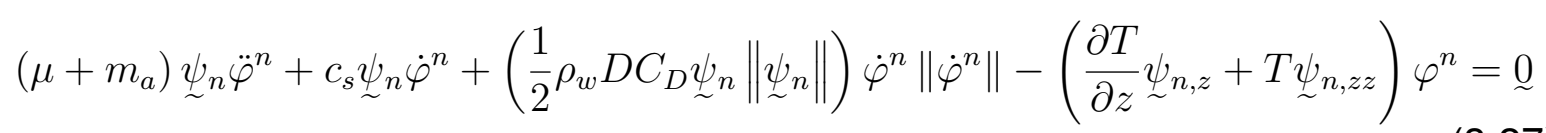

It is necessary to call attention to some notation in eq. 3.27. First, it was used a tensor notation to represent derivatives ${ }^{7}$; last, $Q$ is the vector space null vector. By using the inner product properties, eq. 3.24, and writing $m_{t}=\mu+m_{a}$, it is possible to find an integral form of the modal oscillator; see eq. 3.29.

${ }^{7} \square_{, z}=\square^{\prime}$ and $\square_{, z z}=\square^{\prime \prime}$ 


$$
\begin{aligned}
& \therefore\left\langle\underset{\sim}{0,} \underset{\sim}{\psi_{n}}\right\rangle=0 \\
& \int_{0}^{L} w(z) m_{t}\left(\psi_{n}\right)^{2} \ddot{\varphi}^{n} d z+\int_{0}^{L} w(z)\left[\underset{\sim}{c_{s} \psi_{n}} \dot{\varphi}^{n}+\left(\frac{1}{2} \rho_{w} D C_{D} \underset{\sim}{\psi_{n}}\left\|{\underset{\sim}{\psi}}_{n}\right\|\right) \dot{\varphi}^{n}\left\|\dot{\varphi}^{n}\right\|\right] d z+ \\
& -\int_{0}^{L} w(z) \psi_{n}\left(\frac{\partial T}{\partial z} \psi_{n, z}+T \psi_{n, z z}\right) \varphi^{n} d z=0
\end{aligned}
$$

Finally, using the tension definition, eq. 3.26 and by performing the integration on eq. 3.29, it is possible to write a modal oscillator, as follows:

$$
M_{n} \ddot{\varphi}^{n}+C_{s, n} \dot{\varphi}^{n}+C_{n} \dot{\varphi}^{n}\left\|\dot{\varphi}^{n}\right\|+\left[\eta_{n}+\xi_{n} \cos \left(\omega_{t} t\right)\right] \varphi^{n}=0
$$

in which:

$$
\begin{aligned}
M_{n} & =\int_{0}^{L} m_{t} w(z)\left(\psi_{n}\right)^{2} d z \\
C_{s, n} & =\int_{0}^{L} w(z) c_{s}\left(\psi_{n}\right)^{2} d z \\
C_{n} & =\int_{0}^{L} w(z)\left[\frac{1}{2} \rho_{w} D C_{D}\right]\left(\psi_{n}\right)^{2}\left\|\psi_{n}\right\| d z \\
\eta_{n} & =\int_{0}^{L} w(z)\left\{\gamma \psi_{n, z}+\left[T_{t}-\gamma(L-z)\right] \psi_{n, z z}\right\} \psi_{n} d z \\
\xi_{n} & =\int_{0}^{L} w(z) \frac{E A}{L} A_{t} \psi_{n, z z} \psi_{n} d z
\end{aligned}
$$

Rewriting eq.3.30 in a more convenient form:

$$
\ddot{\varphi}^{n}+2 \zeta_{n} \omega_{N, n} \dot{\varphi}^{n}+\beta_{n} \dot{\varphi}^{n}\left\|\dot{\varphi}^{n}\right\|+\left[\omega_{N, n}^{2}+\hat{\xi}_{n} \cos \left(\omega_{t} t\right)\right] \varphi^{n}=0
$$




$$
\begin{array}{r}
2 \zeta_{n} \omega_{N, n}=\frac{C_{s, n}}{M_{n}} \\
\beta_{n}=\frac{C_{n}}{M_{n}} \\
\omega_{N, n}^{2}=\frac{\eta_{n}}{M_{n}} \\
\hat{\xi}_{n}=\frac{\xi_{n}}{M_{n}}
\end{array}
$$

Regarding eq. 3.31, it is possible to recognize some important parameters. The most important is the modal natural frequency of the $n$-th mode, its eigenvalue, given by $\omega_{N, n}=$ $\sqrt{\frac{\eta_{n}}{M_{n}}}$ in $[\mathrm{rad} / \mathrm{s}]$.

Finally, by doing a change of variable, putting $2 \tau=\omega_{t} t$, it is possible to define a modal damped Mathieu oscillator; see eq. 3.32.

$$
\frac{d^{2} \varphi^{n}(\tau)}{d \tau^{2}}+2 \zeta_{n}^{\star} \omega_{N, n} \frac{d \varphi^{n}}{d \tau}+2 \hat{\beta}_{n} \frac{d \varphi^{n}}{d \tau}\left\|\frac{d \varphi^{n}}{d \tau}\right\|+\left[\delta_{n}+2 \varepsilon_{n} \cos (2 \tau)\right] \varphi^{n}(\tau)=0
$$

in which:

$$
\begin{aligned}
& \zeta_{n}^{\star}=\frac{2 \zeta_{n}}{\omega_{t}} \\
& \hat{\beta}_{n}=\frac{\beta_{n}}{2} \\
& \delta_{n}=\left(\frac{2 \omega_{N, n}}{\omega_{t}}\right)^{2} \\
& \varepsilon_{n}=\frac{2 \hat{\xi}_{n}}{\left(\omega_{t}\right)^{2}}
\end{aligned}
$$

Ramani et al. (2004) shows that it is possible to determine a correction to the Strutt diagram using perturbation techniques, in order to adjust the diagram to the quadratically-damped Mathieu equation; see eq. 3.32. 
If considered $\beta_{n}=0$ and a change of variables given by $\varphi^{n}(\tau)=\vartheta^{n}(\tau) e^{-\zeta_{n}^{\star} \omega_{n} \tau}$ :

$$
\frac{d^{2} \vartheta}{d \tau^{2}}+\left[\delta_{n}^{\star}+2 \varepsilon_{n} \cos (2 \tau)\right] \vartheta^{n}=0, \quad \delta_{n}^{\star}=\delta_{n}-\left(\zeta_{n}^{\star} \omega_{N, n}\right)^{2}
$$

The classical Mathieu-Hill is reached in eq.,3.33 when the quadratically-damping term is neglected. On the other hand, the linear damping term is considered, being possible to analyze a linear damped Mathieu equation using a classical Strutt diagram; see Meirovitch (2003). In the new variable, $\vartheta^{n}$, the stability chart is dislocated to the right in the $\delta^{\star}$-parameter. 


\section{Chapter 4}

\section{Experimental Campaign}

\subsection{Experiments in water: a brief account on previous experiments at IPT}

In this section, it shall be presented an useful guide in order to demonstrate how a small-scale model of a real riser was designed using similitude properties. As it has been already mentioned, the present work is a direct consequence from the R\&D project "Non-Linear Dynamics of Risers: non-linear interactions of hydro-elastic and contact nature", sponsored by Petrobras; see the preface on pg. xxi for further acknowledgments.

\subsubsection{Froude similitude}

In order to perform the experimental tests, it was developed a similitude algorithm in order to represent a regular sin diameter Steel Catenary Riser (SCR) as a small-scale model, for laboratory practical uses. After some analysis, a collection of dimensionless control parameters was proposed to analyse the non-linear dynamics of risers (on still water), including non-linearities of geometric, hydro-elastic and contact nature; see Table 4.1. Unfortunately, it is impossible to reach a model in which both Reynolds $(\mathrm{Re})$ and Froude (Fr) similarities are satisfied. Thereby, the Froude scale was chosen in order to simulate the dynamical load due to vertical motions imposed at the top of the riser, mainly caused by the effect of gravity waves on the floating structure to which the riser is fixed. 
Table 4.1: Dimensionless parameters.

\begin{tabular}{ccc}
\hline Number & Symbol & Representation \\
\hline Froude number & $F r=\frac{\omega A}{\sqrt{g L}}$ & Dynamic motion in waves \\
Reynolds number & $R e=\frac{U D}{\nu}$ & Inertial forces vs. viscous forces \\
Strouhal number & $S t=\frac{f_{s} D}{U}$ & Vortex shedding frequency \\
Keulegan-Carpenter number & $K C=\frac{2 \pi A}{D}$ & Inertial forces vs. drag forces \\
Structural damping & $\zeta=\frac{\xi}{\xi_{c r}}$ & Linear structural damping \\
Reduced velocity & $V_{R}=\frac{U}{f_{N} D}$ & Normalized velocity in VIV \\
Reduced shedding frequency & $f_{s}^{\star}=\frac{f_{s}}{f_{N}}=S t V_{R}$ & Vortex shedding normalized frequency \\
Reduced mass & $m^{\star}=\frac{m}{m_{d}}$ & Riser mass vs. displaced mass \\
Added mass & $C_{a}=\frac{m_{a}}{m_{d}}$ & Added mass vs. displaced mass \\
Bending stiffness & $K_{f}=\frac{\lambda_{f}}{L}$ & Bending stiffness vs. geometrical stiffness \\
Axial stiffness & $K_{a}=\frac{E A}{T}$ & Axial vs. geometrical stiffness \\
\hline
\end{tabular}

Source: Extracted from Pereira et al. (2012)

The Froude scale parameter, $\lambda_{F r}$, sets a relationship between the operational riser and the small-scale model with the quantity features presented on Table $4.2^{1}$. The scale chosen was $\lambda_{F r}=1: 100$ which leads to non-practical external diameter order of about $\mathcal{O}=2 \mathrm{~mm}$. The solution proposed by Pereira et al. (2012) was to increase the external diameter, keeping constant three physical quantities from the scaled-model; they are:
i. Axial stiffness: $E A$;
ii. Flexural length: $\lambda_{f}$;
iii. Added mass coefficient: $C_{a}$ or $a$.

In order to achieve such small-scale model, it was used an iterative algorithm in which the external and internal diameters of a silicon tube were given as known and the method would evaluate what would be the necessary model density.

Table 4.2: Quantities feautures and Froude similarity.

\begin{tabular}{ccc}
\hline Quantity & Physical dimension & Scale \\
\hline Length & $L$ & $\lambda_{F r}$ \\
Mass & $M$ & $\frac{\rho_{M}}{\rho}\left(\lambda_{F r}\right)^{3}$ \\
Time & $T$ & $\left(\lambda_{F r}\right)^{1 / 2}$ \\
\hline \multicolumn{2}{c}{ Source: Extracted from Pereira et al. $(2012)$}
\end{tabular}

\footnotetext{
${ }^{1}$ It is quite easy to reach at this result. Considering $F r$ as the Froude number of the operational riser and $F r_{M}$, of the small-scale model: $F r=\omega A / \sqrt{g L}=\omega_{M} A_{M} / \sqrt{g_{M} L_{M}}=F r_{M}$. Choosing $L_{M}=\lambda_{F r} L$ and regarding that the gravity is kept constant, it will be reached at: $\omega_{M}=\left(\lambda_{F r}\right)^{-1 / 2} \omega \Longleftrightarrow T_{M}=$ $\left(\lambda_{F r}\right)^{1 / 2} T$. Therefore, $M_{M}=\rho_{M} L_{M}^{3}=\left(\lambda_{F r}\right)^{3}\left(\rho_{M} / \rho\right) M$.
} 
The material needed to fill the silicon tube should be very dense, but with some restrictions, e.g., non-toxic, accessible and reusable. The solution found was a silicon tube filled with stainless steel micro-spheres. The micro-spheres used were chosen regarding the effective density of the material, including the voids filled with air among the micro-spheres, leading to $\varnothing 0.5 \mathrm{~mm}$ to the micro-spheres. A data summary with all mechanical properties of the $\mathrm{SCR}^{2}$ chosen, the scaled model, the small-scale model with its external and internal diameter increased and the "as built" experimental model is shown in Table 4.3.

Table 4.3: Data summary: SCR, scaled model, designed small-scale model and "as built".

\begin{tabular}{ccccc}
\hline Data & 8" SCR & Scaled & Small-scale & As built \\
\hline Internal diameter $(\mathrm{mm})$ & 182.58 & 1.826 & 15.800 & 15.8 \\
External diameter $(\mathrm{mm})$ & 219.10 & 2.191 & 22.200 & 22.2 \\
Weight in water $(\mathrm{N} / \mathrm{m})$ & 726.42 & 0.726 & 7.308 & 7.308 \\
Axial stiffness, $E A(\mathrm{kN})$ & $2.36 E+06$ & 2.362 & 1.910 & $1.0-1.6$ \\
Bending stiffness, $E I\left(\mathrm{Nm}{ }^{2}\right)$ & $1.20 E+07$ & $1.20 E-03$ & $8.86 E-02$ & $5.60 E-02$ \\
Flexural length, $\lambda_{f}(\mathrm{~mm})$ & 7.139 & 71.0 & 61.0 & 49.0 \\
Added mass coeff, $C a=m_{a} / m_{d}$ & 0.522 & 0.522 & 0.520 & 0.520 \\
\hline
\end{tabular}

Source: Extracted from Pereira et al. (2012)

The physical model may be regarded in Figure 4.1 and the reader may find interesting the optical targets fixed on the model; their purpose will be explained later.

Figure 4.1: A segment of the "as built" model: a silicon tube filled with stainless steel micro-spheres with optical targets fixed on the model for displacement measuring purposes.

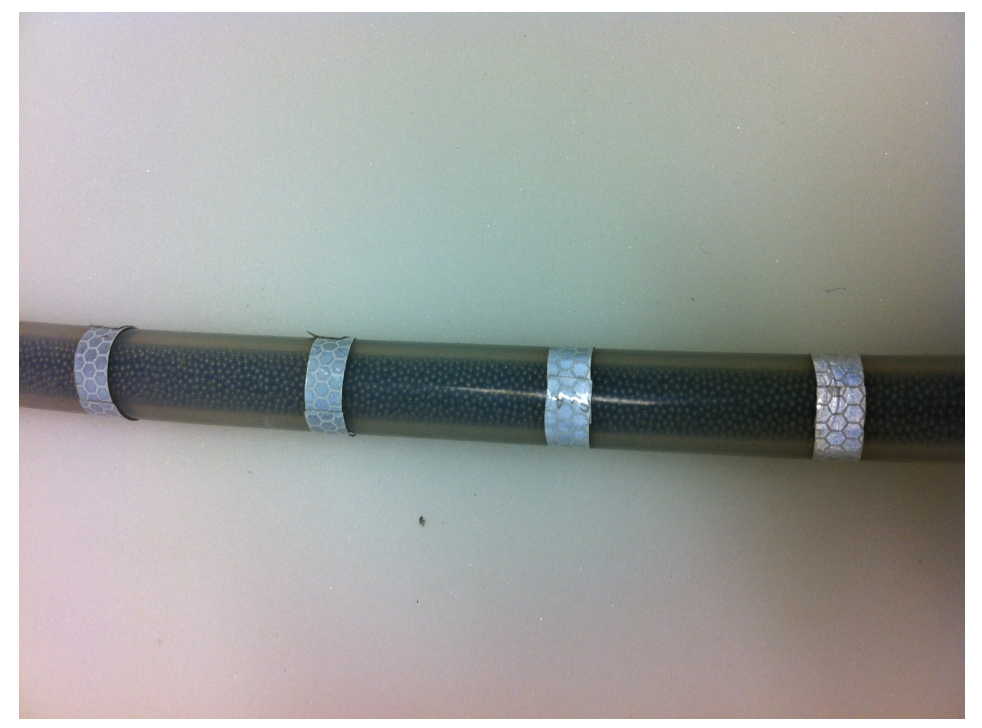

To the knowledge of the author, there are not available publications of an experimental

\footnotetext{
${ }^{2}$ The flexible vertical scaled riser model is the same model used in the SCR.
} 
inroad with a small-scale riser model so sophisticated, keeping a similitude with a real operational riser. The silicon tube chosen presented a viscous-elastic rheological behavior, see Figure 4.2, something that was not expected a priori, since the majority of experimental models in this field are considered to be linear-elastic.

Figure 4.2: Viscous-elastic behavior of experimental model under cyclic tension test. Maximum engineering deformation of $\epsilon \approx 0.1 \%$ at $f=1.5 \mathrm{~Hz}$.

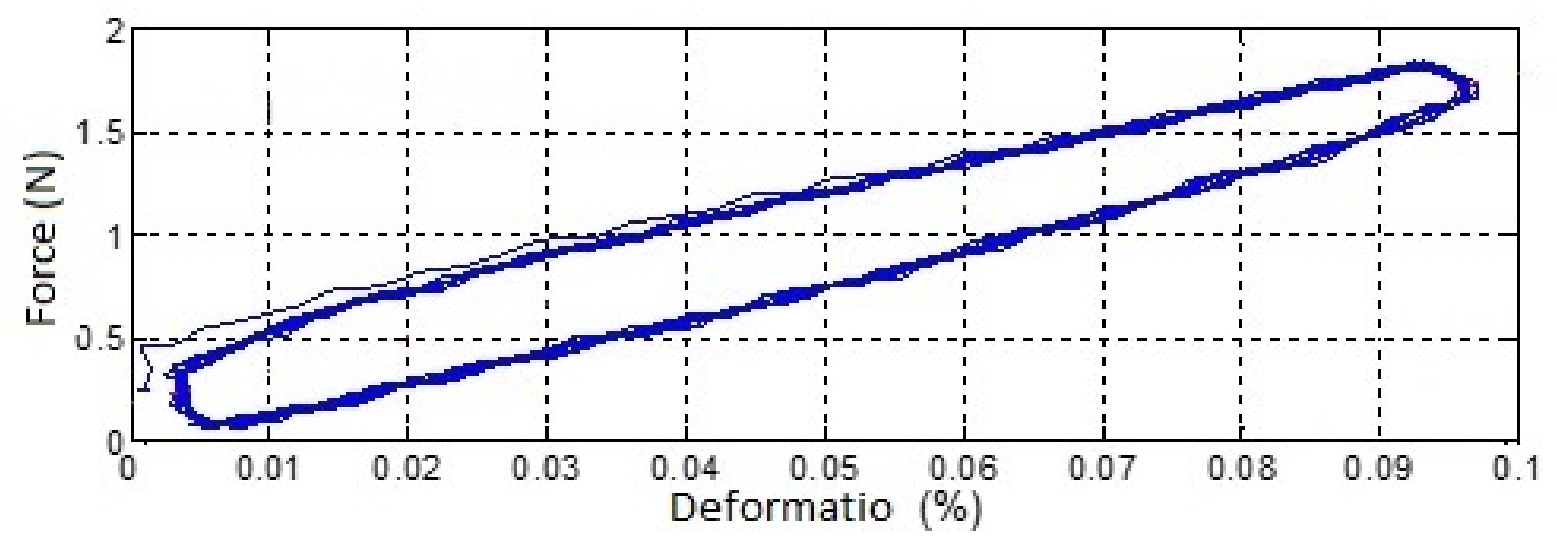

As much as the small-scale model aims at an operational riser, they will not establish a full similarity to each other, inasmuch as it is impossible to match the Re using the Froude scale adopted. If the total length of the small-scale model tested is considered, $L=2602 \mathrm{~mm}$, see Table 4.4, which would be equivalent to a depth of approximately $\sim 260 \mathrm{~m}$ on the ocean. It would not be considered as very deep waters. Such details would cause a raid in some readers who would argue that the model does not represent a SCR, consequently a flexible vertical riser, and, in fact, the model does not; at least not strictly. Nevertheless, the small-scale model is as close as possible of a real riser, providing experimental results at a laboratory facility that could even be used as benchmarking for other analytical, semi-analytical or numerical models. Thereby, in order to be more consistent, it will be understood from now on that the present work is focused on the dynamics of a flexible vertical cylinder, which was designed and built inspired in a real riser with a good degree of the Froude similitude linking them. 
Table 4.4: Mechanical properties from the experimental model of flexible vertical cylinder model in water.

\begin{tabular}{cc}
\hline Property & Value \\
\cline { 2 - 2 } Unstretched length, $L_{0}$ & $2552 \mathrm{~mm}$ \\
Stretched length, $L$ & $2602 \mathrm{~mm}$ \\
Imeersed length, $L_{i}$ & $2257 \mathrm{~mm}$ \\
Mass ratio parameter, $m^{\star}$ & 3.48 \\
Aspect ratio, $L_{i} / D$ & 102 \\
$L / D$ & 117 \\
Static tension at the top, $T_{t}$ & $40 \mathrm{~N}$ \\
Immersed linear weight, $\gamma_{i}$ & $7.31 \mathrm{~N} / \mathrm{m}$ \\
Linear mass, $\mu$ & $1.14 \mathrm{~kg} / \mathrm{m}$ \\
\hline Source: & Extracted from LIFE\&MO (2012d) \& Franzini et al. (2014)
\end{tabular}

\subsubsection{Experimental set-up: a brief description}

The model designed was first tested at the NDF water channel and at the CH-TPN immersed in water and in air, in order to be familiar with the experimental assembling, challenges and to assimilate as much knowledge as possible about the model; see LIFE\&MO (2012a, 2012b). The vertical model was assembled at the NDF water channel and some preliminary experimental analyses were made at that time. A preparation for the experimental set-up that would be mounted on the Towing Tank from IPT.

Regarding now the experimental set-up at IPT facility, it is necessary identify some basic general data about the methodology used. First, using the previous analyses made with the model at NDF and TPN facilities, it was determined that the phenomena studied would concentrate their frequency spectra within a range between $0.5 \mathrm{~Hz}$ up-tp $6.0 \mathrm{~Hz}$. Overestimating the sampling frequency, it was chosen the value of $F_{s}=60 \mathrm{~Hz}$, which it is not the best suitable choice, since the electric power supply has the same frequency, the multiplicity by $1 / 3$ might promote some truncate error on numerical algorithms and the next power-of-two number is very close.

For the purpose of measuring the elastica dynamics, it was used an optical tracking system, by Qualisys ${ }^{\mathrm{TM}}$, which captures the displacement of each discrete optical target attached to the experimental model. Two aerial cameras were used to measure the elastica displacement of the above water model segment, and more 6 submerged cameras in order to capture the elastica under water; see Figures 4.3-4.4. Besides, it is possible to mention that the accuracy of the optical system is about $\pm 0.1 \mathrm{~mm}$. The relative flow around the model was created by the moving towing tank carriage, with the velocity controlled. 
Figure 4.3: Vertical model. Note: reflexive targets attached to the model in order to capture its displacement.

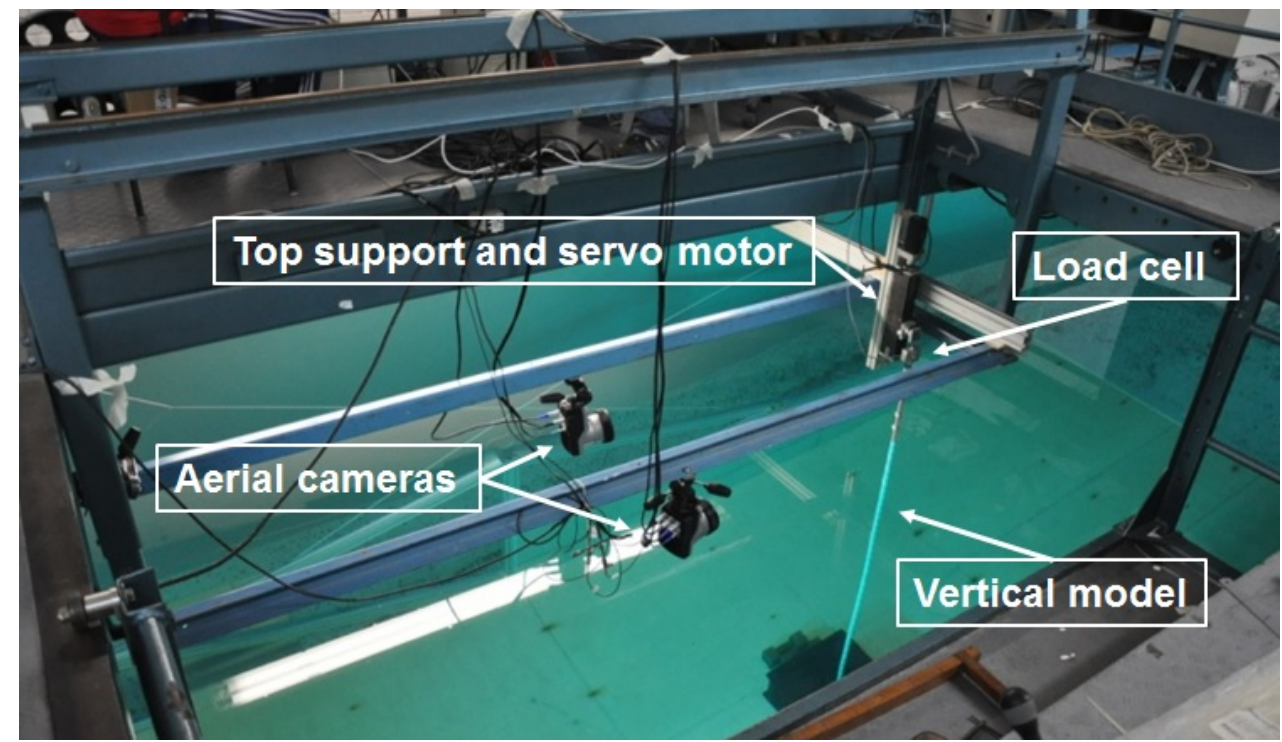

Source: Extracted from LIFE\&MO (2012d).

Figure 4.4: Submerged optical cameras: measuring displacement. Note: model launched in "catenary" configuration.

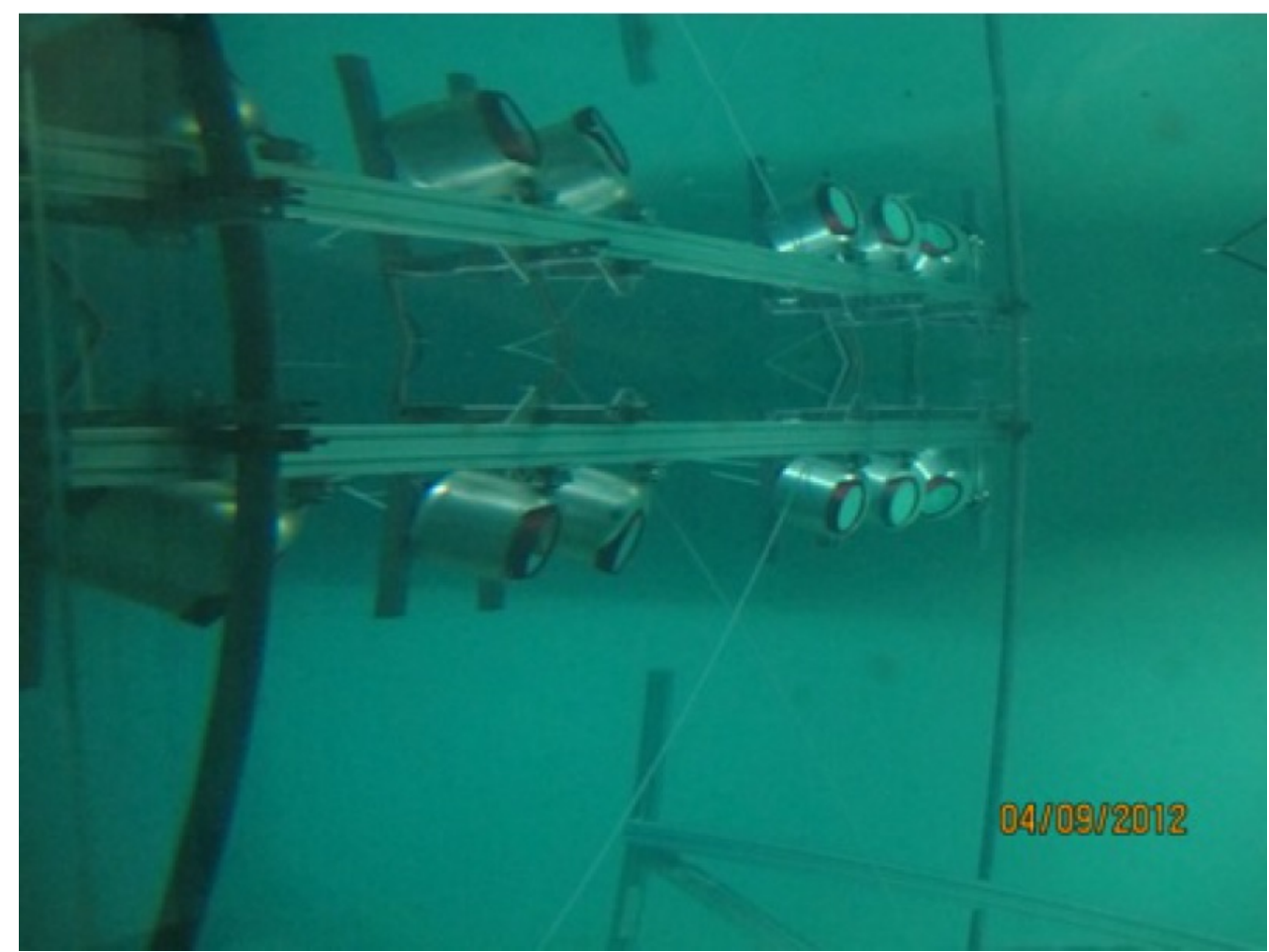

Source: Extracted from LIFE\&MO (2012c).

In addition to the optical cameras, an axial load cell was placed at top of the model in order to record any tension fluctuation. Along with the load cell, a controlled servo-motor was placed on the top support in order to simulate the effects of floating vessels under 
the action of gravitational waves; see Figure 4.5. The servo-motor induced heave motion according to previously established frequencies: $f_{t}: f_{N, 1}=1: 3, f_{t}: f_{N, 1}=1: 2$, $f_{t}: f_{N, 1}=2: 3, f_{t}: f_{N, 1}=1: 1, f_{t}: f_{N, 1}=2: 1, f_{t}: f_{N, 1}=3: 1 \& f_{t}: f_{N, 1}=3: 2$. From now in this work will be considered only the cases: $f_{t}: f_{N, 1}=1: 1, f_{t}: f_{N, 1}=2: 1$ \& $f_{t}: f_{N, 1}=3: 1$.

Figure 4.5: Servo driver and load cell with the model in vertical configuration at IPT.

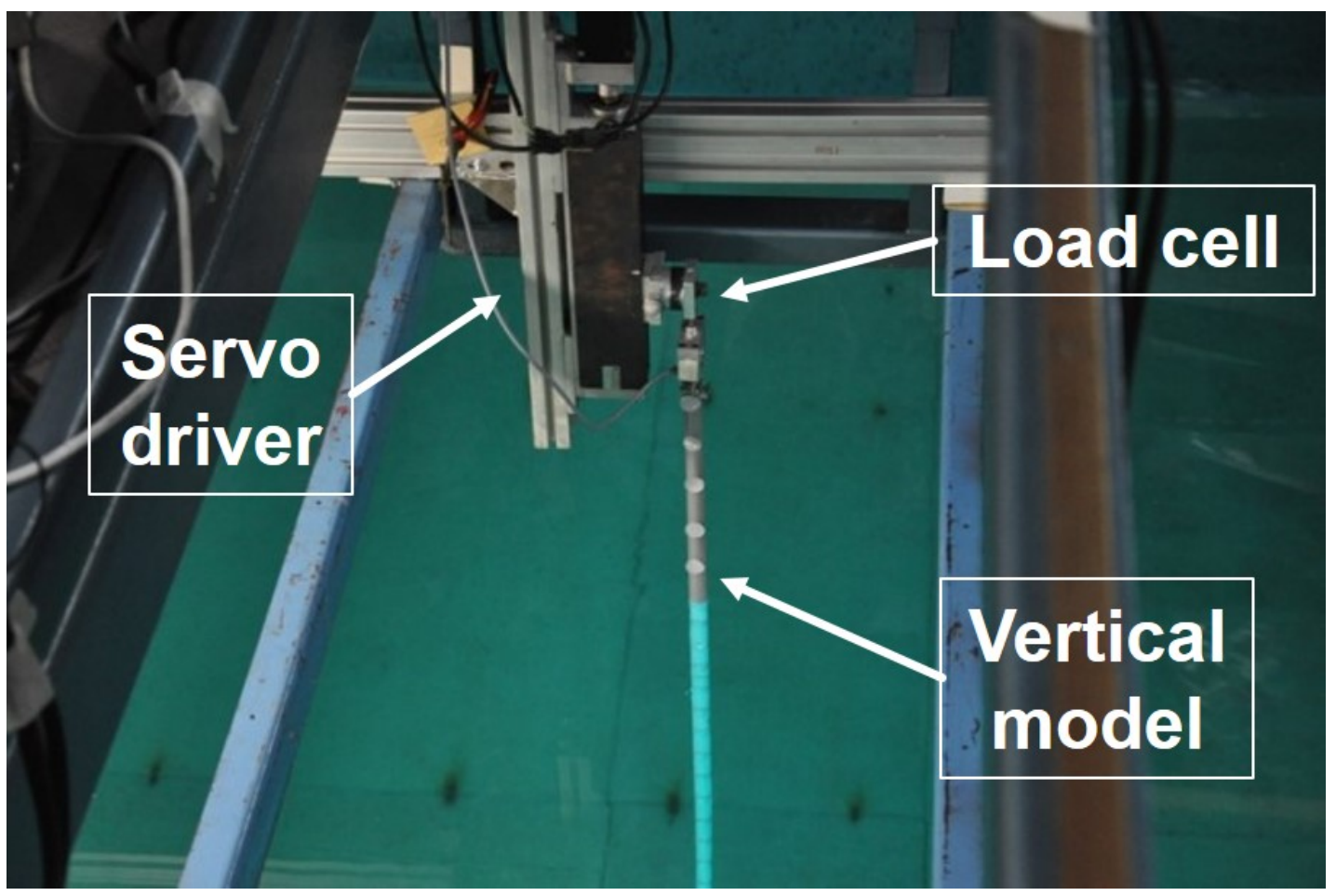

Source: Adapted from LIFE\&MO (2012d) \& Pesce (2013).

It is noteworthy to regard the detailed placement of the reflexive targets; see Figure 4.6. The reflexive target near the water surface was poorly detectable by the monitoring system, given its problematic intermittent dynamics in-and-out of water. Finally, the schematic models of both VIV and heave experimental set-up conditions and some parameters are given in Figure 4.7a-b. 
Figure 4.6: Vertical model. Note: reflexive targets attached to the model in order to capture its displacement.

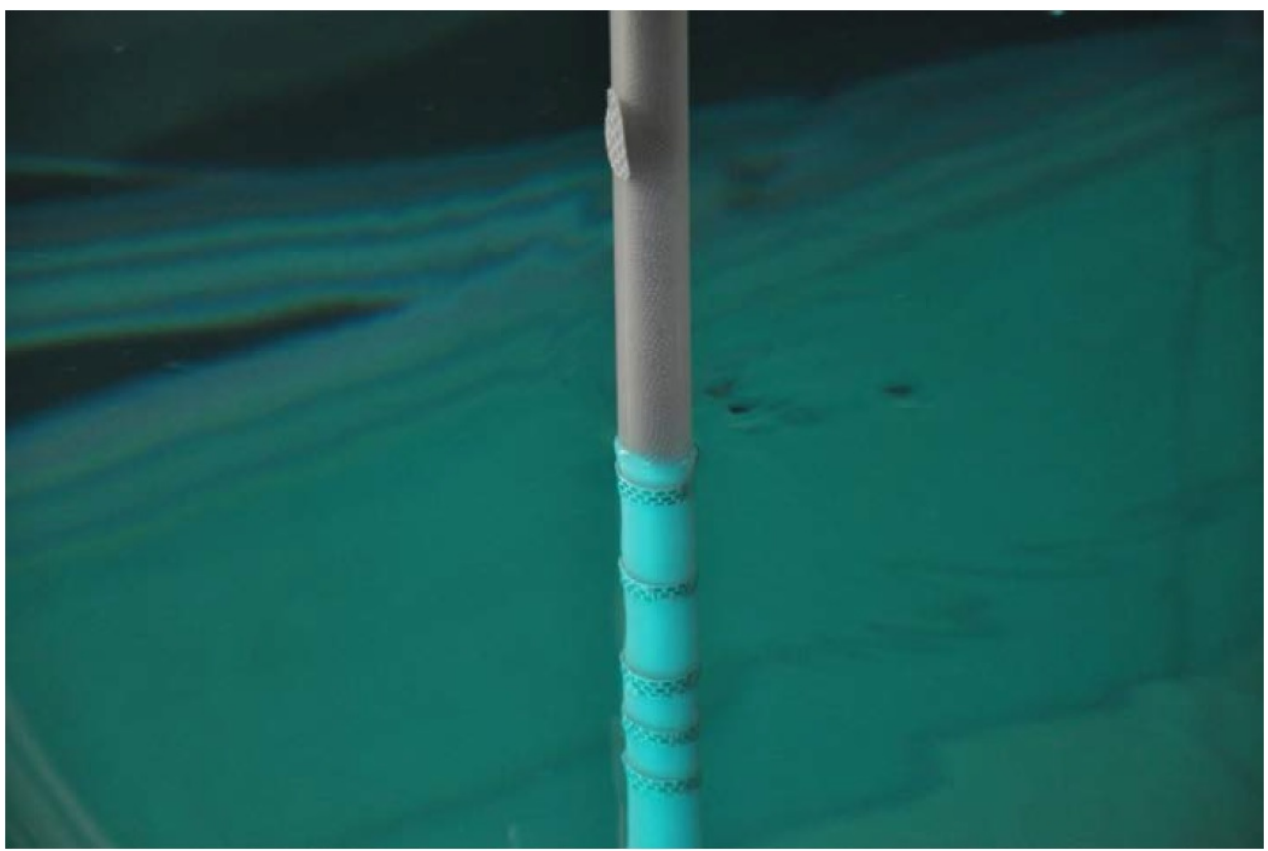

Source: Extracted from LIFE\&MO (2012d).

Figure 4.7: Schematic experimental set-up.

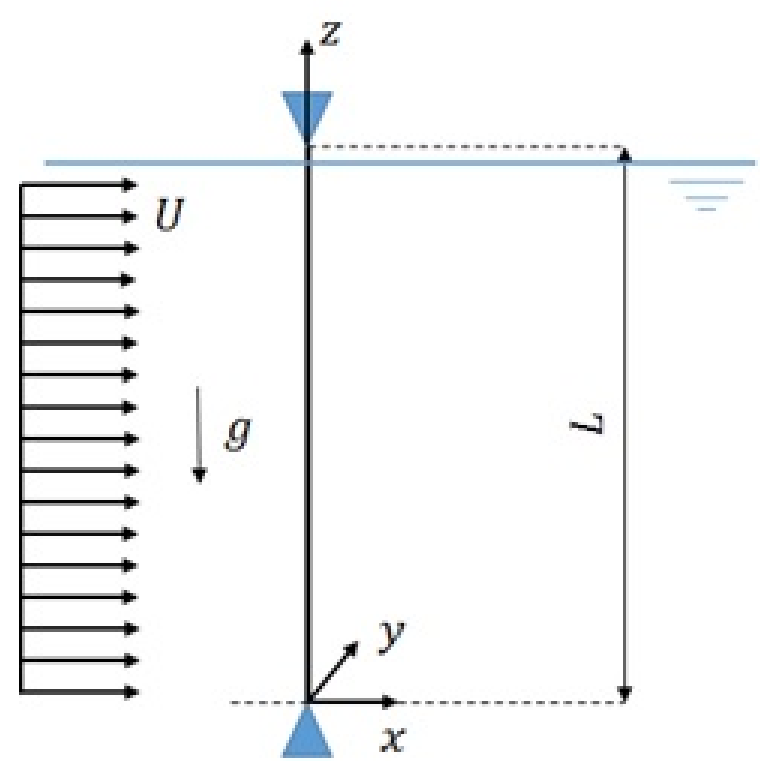

(a) VIV schematic set-up.

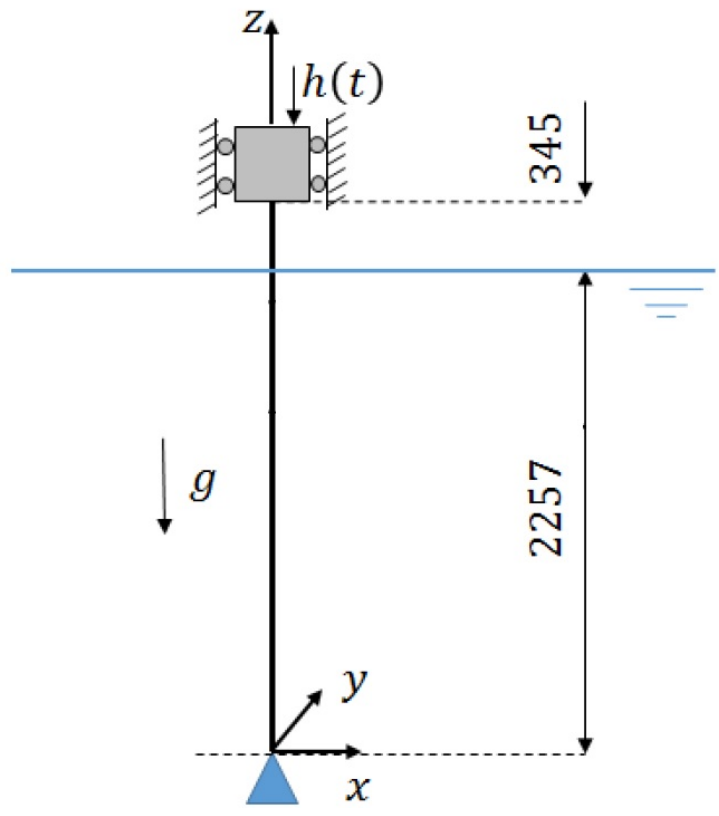

(b) Heave schematic set-up. Distances in $\mathrm{mm}$. 


\subsection{New experiments in air}

The experiments carried out in air were conducted using the same small-scale model aforementioned. The preparation of the physical model may be seen in Figs.4.8-4.9.

Figure 4.8: Experimental model used in air. Unstretched length, $L_{0}=2613 \mathrm{~mm}$.

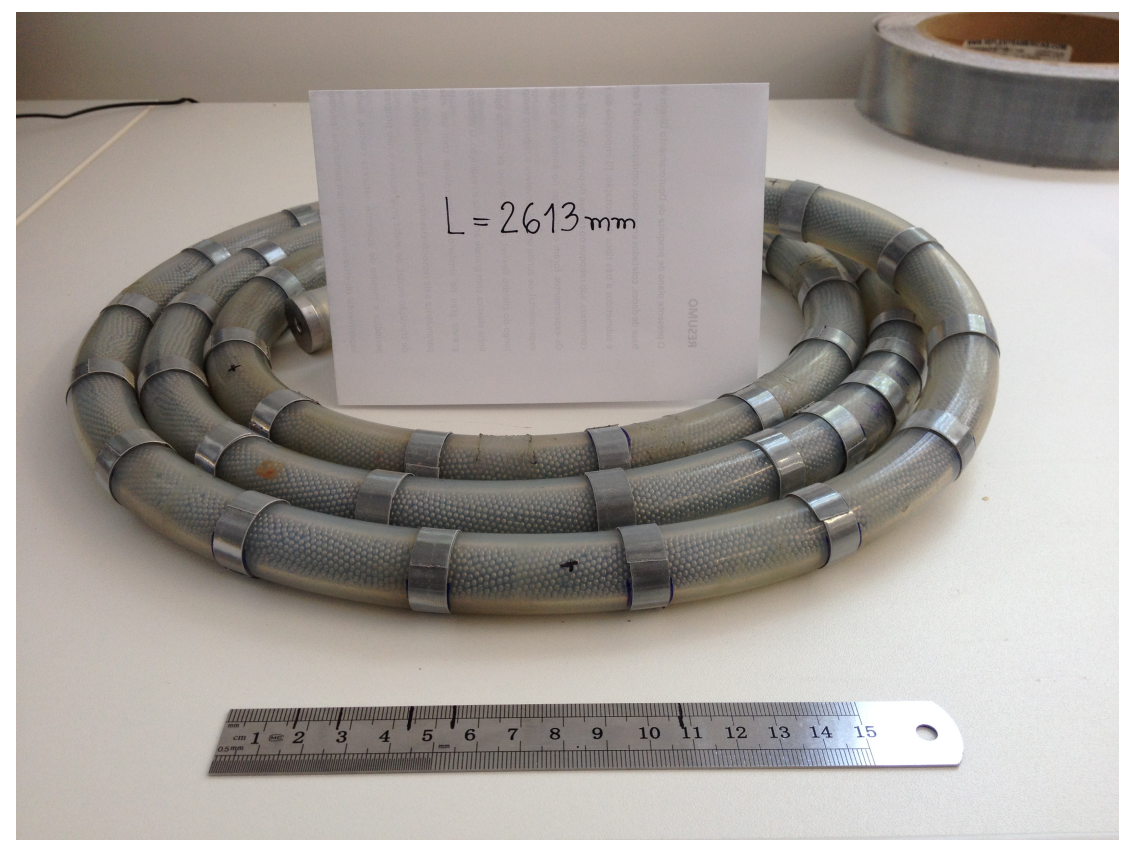

Figure 4.9: Experimental model used in air. Detail to the discretization of the model using optical targets and the filling of micro-spheres.

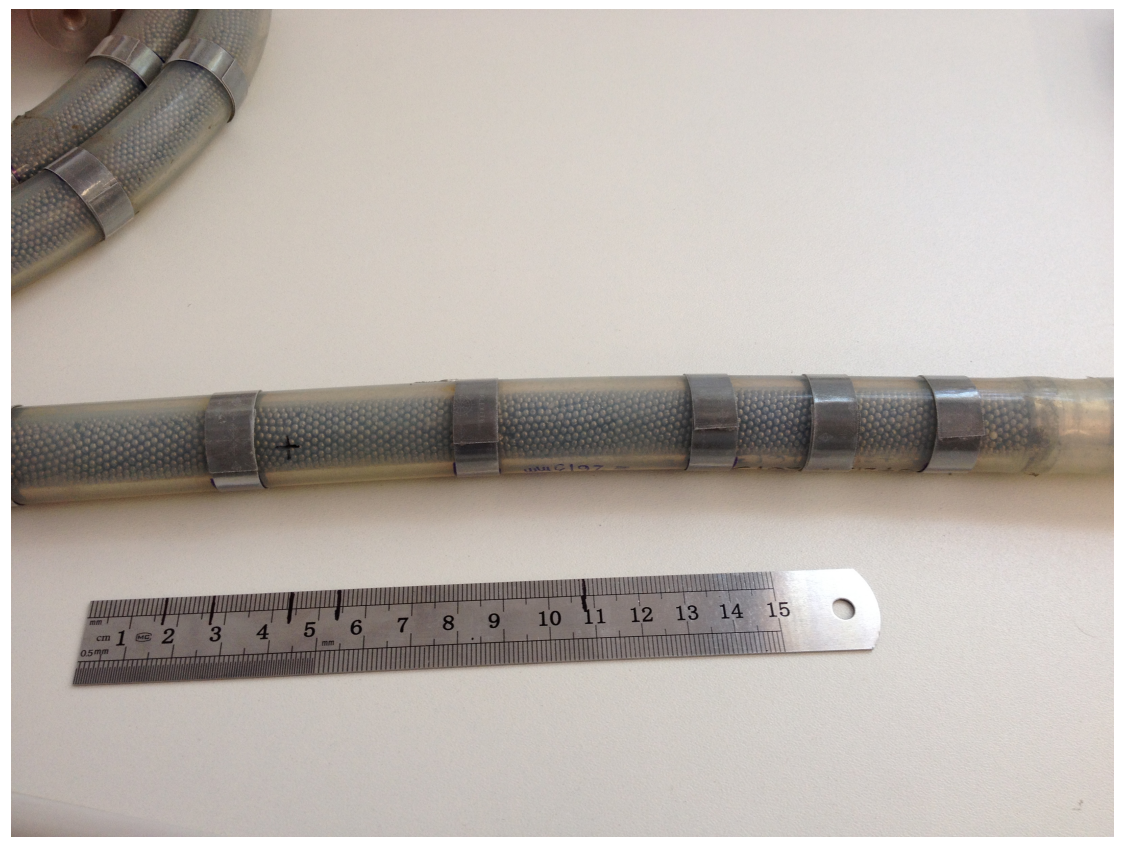

The total amount of optical targets in the discretization is 54, equidistant from each other, except by a refined discretization made on both ends of the cable. 
The structure in which the cable was tested was installed at TPN facility. The structure is composed of aluminum profile bars, attached to the TPN facility structure using C-clamps. The base of the model was composed of a structure of profile bars and a load cell with 6 degrees-of-freedom; see Figs.4.10-4.11.

Figure 4.10: Superior part of the structure assembled and base in which the model is fixed.

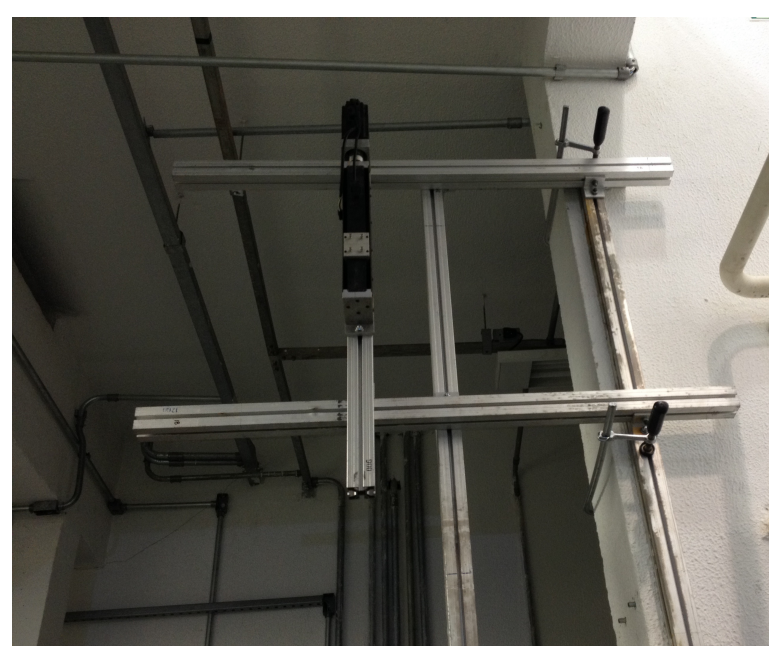

(a) Frontal view of the upper part of the structure with servo driver installed.

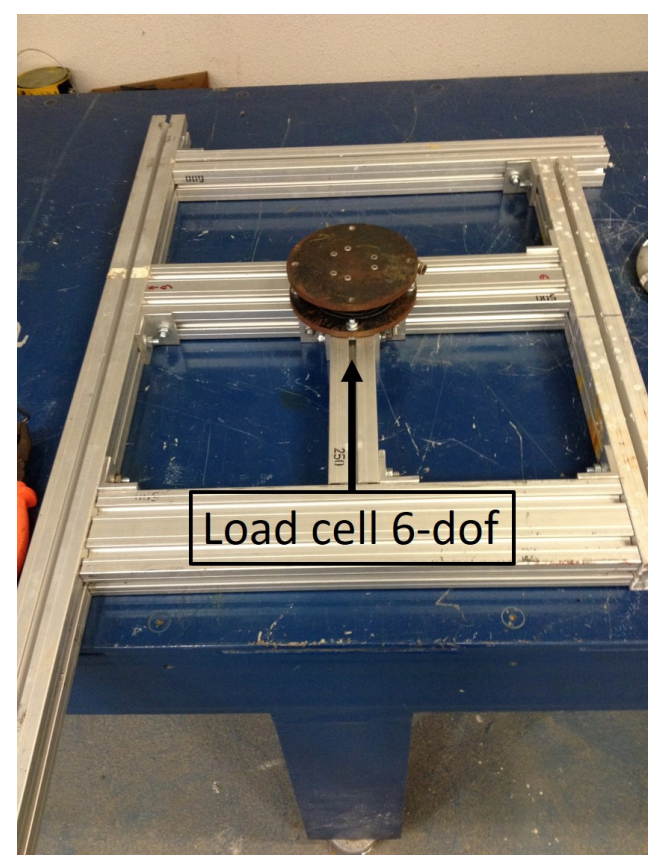

(b) The structure base.

Figure 4.11: Lateral view of the structure.

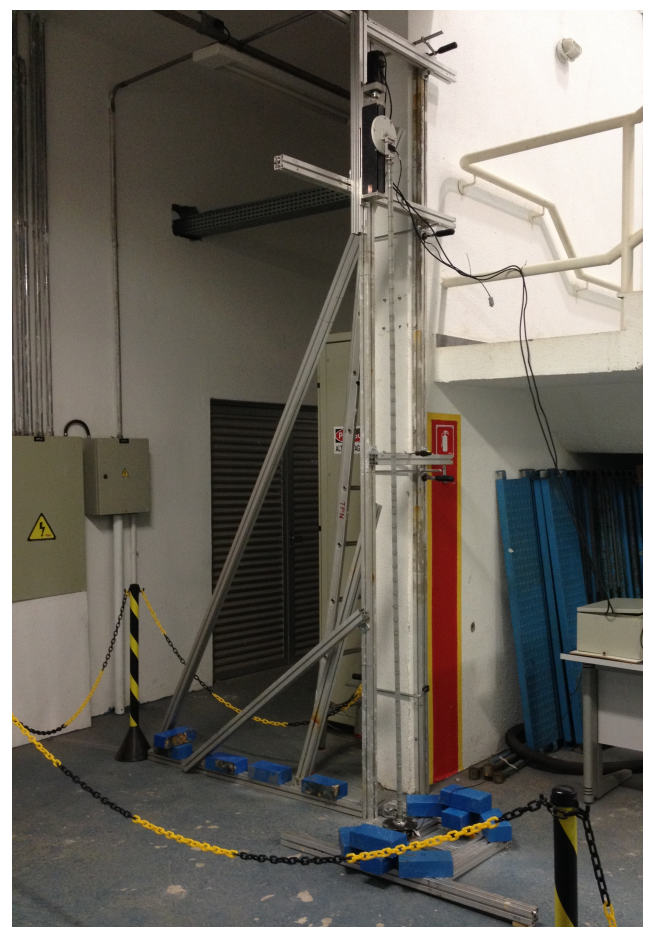


The final assembled structure is presented in Fig.4.12. Besides the structure presented, an optical tracking system using 3 cameras was used; see Fig.4.13. Finally, the axis defined on the experimental tests are shown; see Fig.4.14.

Figure 4.12: The final structure with model suspended.

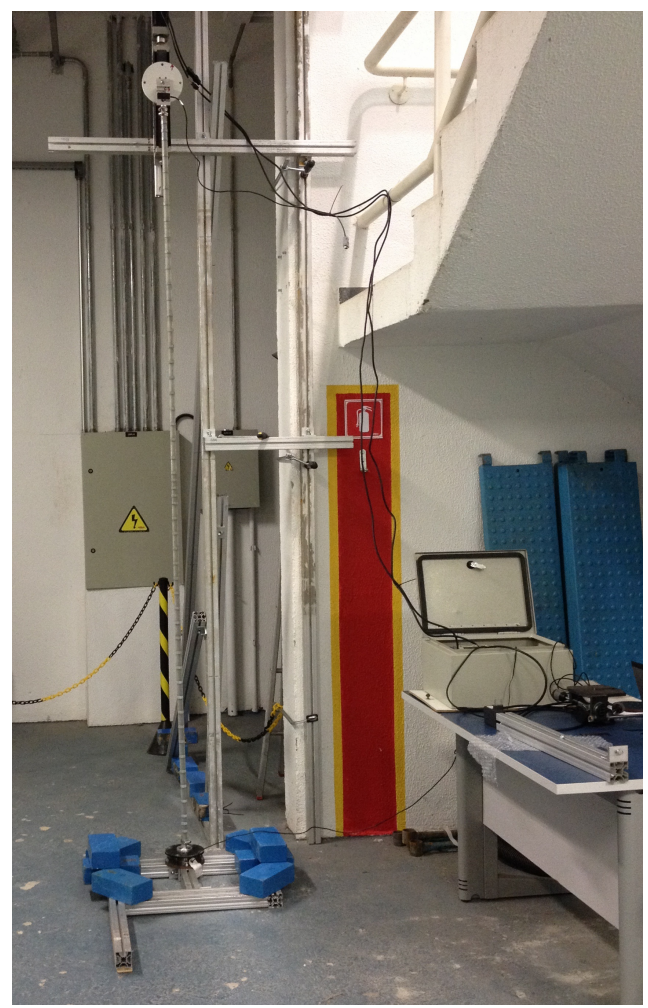

Figure 4.13: Set of optical tracking cameras positioned in front of the model.

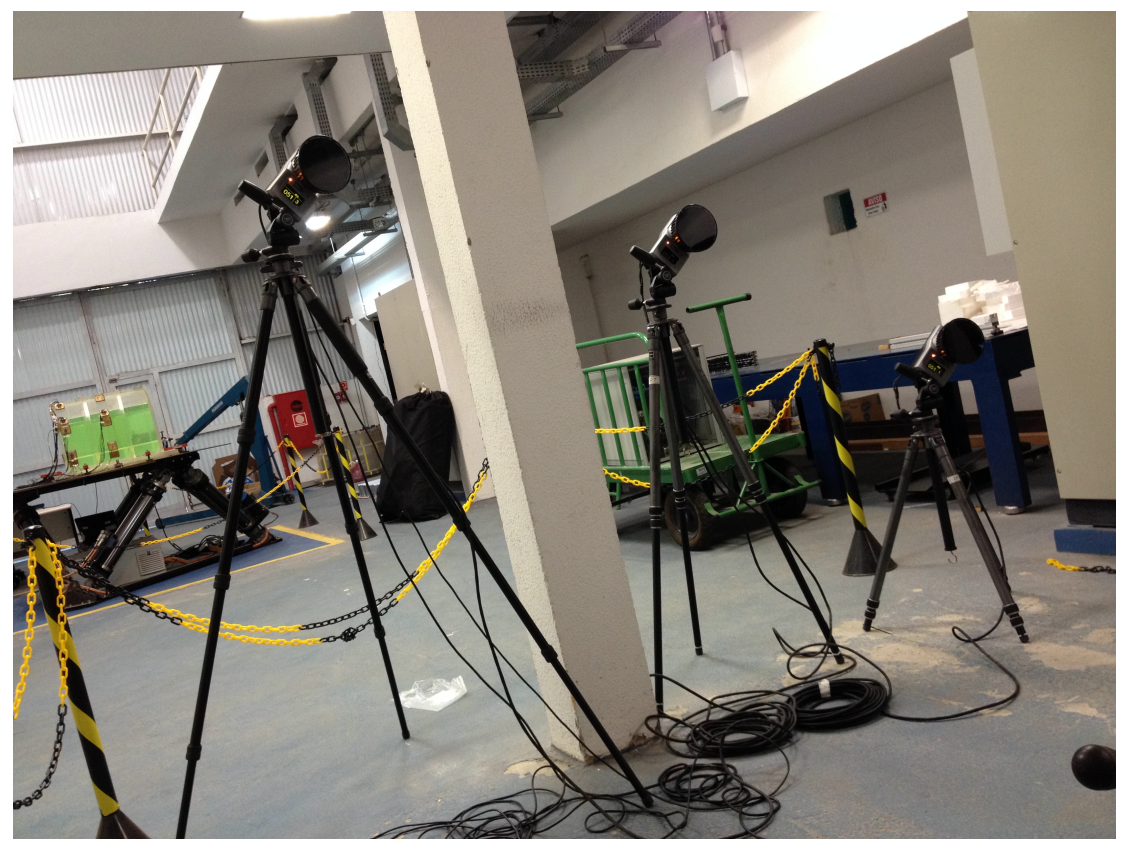


Figure 4.14: The final structure assembled with model suspended further detailed.

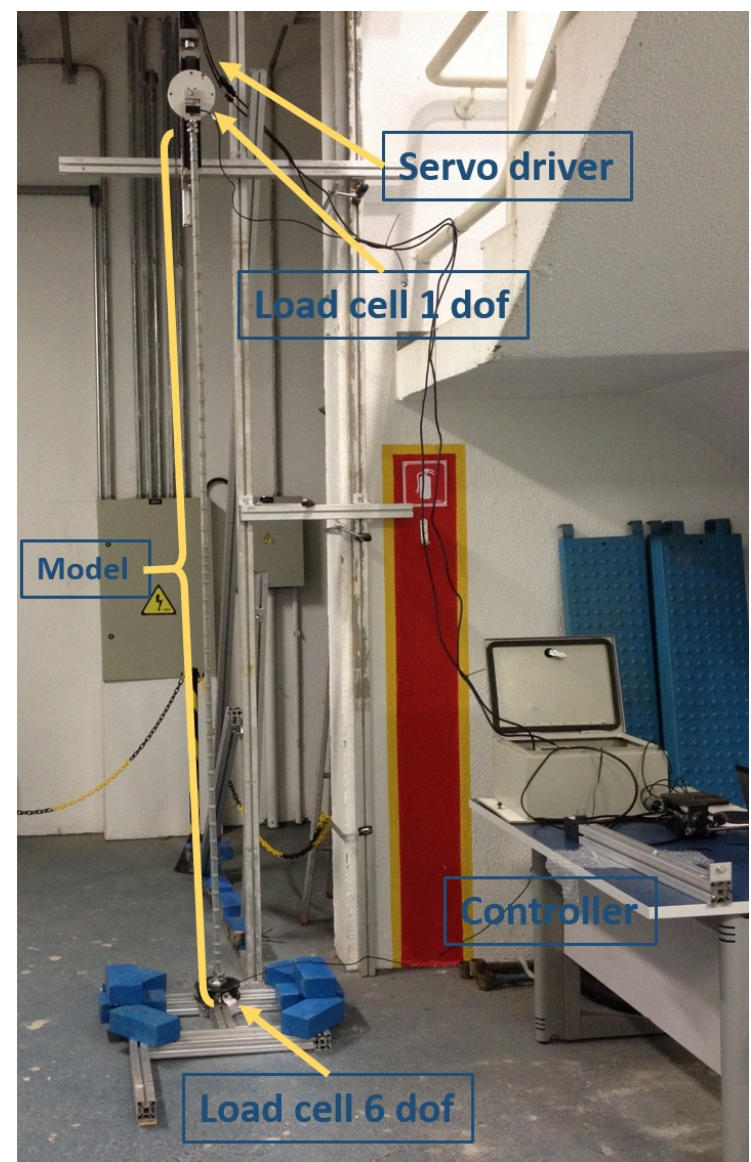

(a) Assembled structure with the as-built model.

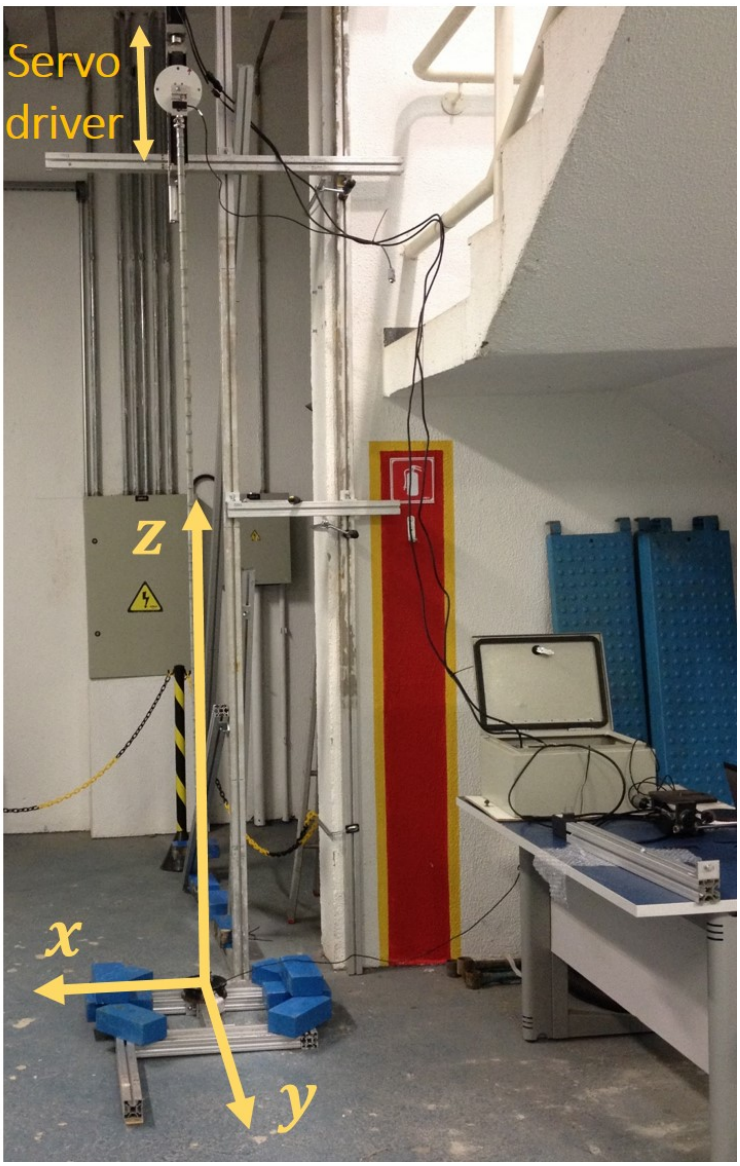

(b) Cartesian axis defined on the experimental tests.

Some properties of the flexible vertical cylinder in air experimental set-up are summarized in Table 4.5 .

Table 4.5: Mechanical properties from the experimental model of flexible vertical cylinder model in air.

\begin{tabular}{cc}
\hline Property & Value \\
\hline Unstretched length, $L_{0}$ & $2613 \mathrm{~mm}$ \\
$L_{0} / D$ & 118 \\
Static tension at the top, $T_{t}$ & $47.36 \mathrm{~N}$ \\
Linear weight, $\gamma$ & $11.13 \mathrm{~N} / \mathrm{m}$ \\
Linear mass, $\mu$ & $1.14 \mathrm{~kg} / \mathrm{m}$ \\
Sampling frequency, $F_{s}$ & $100 H z$ \\
\hline
\end{tabular}




\section{Chapter 5}

\section{Experimental Analysis: methodology and results}

The following chapter is dedicated to explore some analyses that have been made, using some of the theoretical points presented on the Chapter 3 . The text will be organized on four sections which are dedicated to:

1. Modal representation;

2. Imposed heave and modal Mathieu instability;

3. Experimental tests with flexible vertical cylinder in air:

(a) Free-Decay test and structural damping;

(b) Comparison to tests in water in order to estimate modal hydrodynamic coefficients.

\subsection{Modal representation}

Previously, two simplified mathematical models have been presented which lead to two different eigenfunction bases: the classical vibrating string and the vertical string, reaching trigonometric and Bessel-type modes, respectively. Which modal base represents the experimental elastica better?

\subsubsection{Imposed heave motion at the top}

In this section, the effects of imposed heave on the structural dynamics will be analysed. The following cases will be considered:

i. $f_{t}: f_{N, 1}=1: 1$; 
ii. $f_{t}: f_{N, 1}=2: 1$;

iii. $f_{t}: f_{N, 1}=3: 1$.

Initially, observing the heave imposed at $f_{t}: f_{N, 1}=1: 1$, see Figure $5.1 \mathrm{a}-\mathrm{b}$, it is possible to regard some interesting phenomena.

Figure 5.1: Scalograms of imposed heave $-f_{t}: f_{N, 1}=1: 1$.

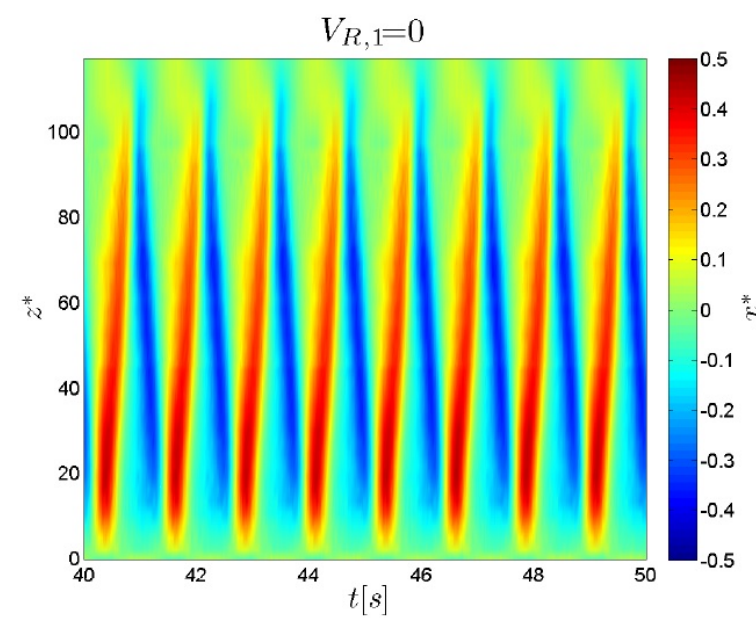

(a) Scalogram of $x^{\star}$ displacement.

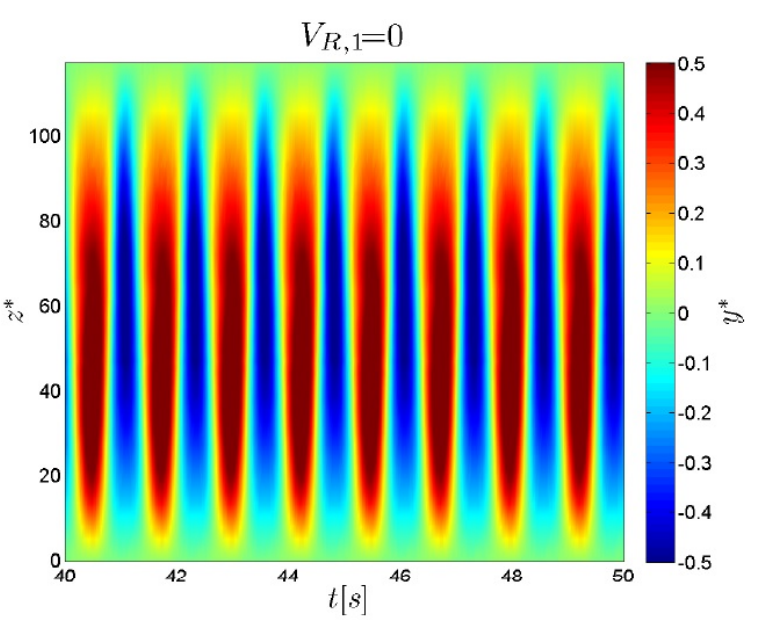

(b) Scalogram of $y^{\star}$ displacement.

Source: Extracted from LIFE\&MO (2012d)

Keeping in mind it is a heave test, it could be asked why there is a difference between directions $x$ and $y$, inasmuch as the cylinder has a circular section. Considering now the PSD of the in-line motion, the one in which occurred the travelling wave, see Figure 5.2, shows that there is a main frequency on the response and the following harmonics are present, but with less energy. It is interesting to think the experimental model is similar to a piano/harp string which presents a proper energy distribution on each harmonic, as the experimental apparatus has its own timbre. 
Figure 5.2: PSD of imposed heave $-f_{t}: f_{N, 1}=1: 1$.

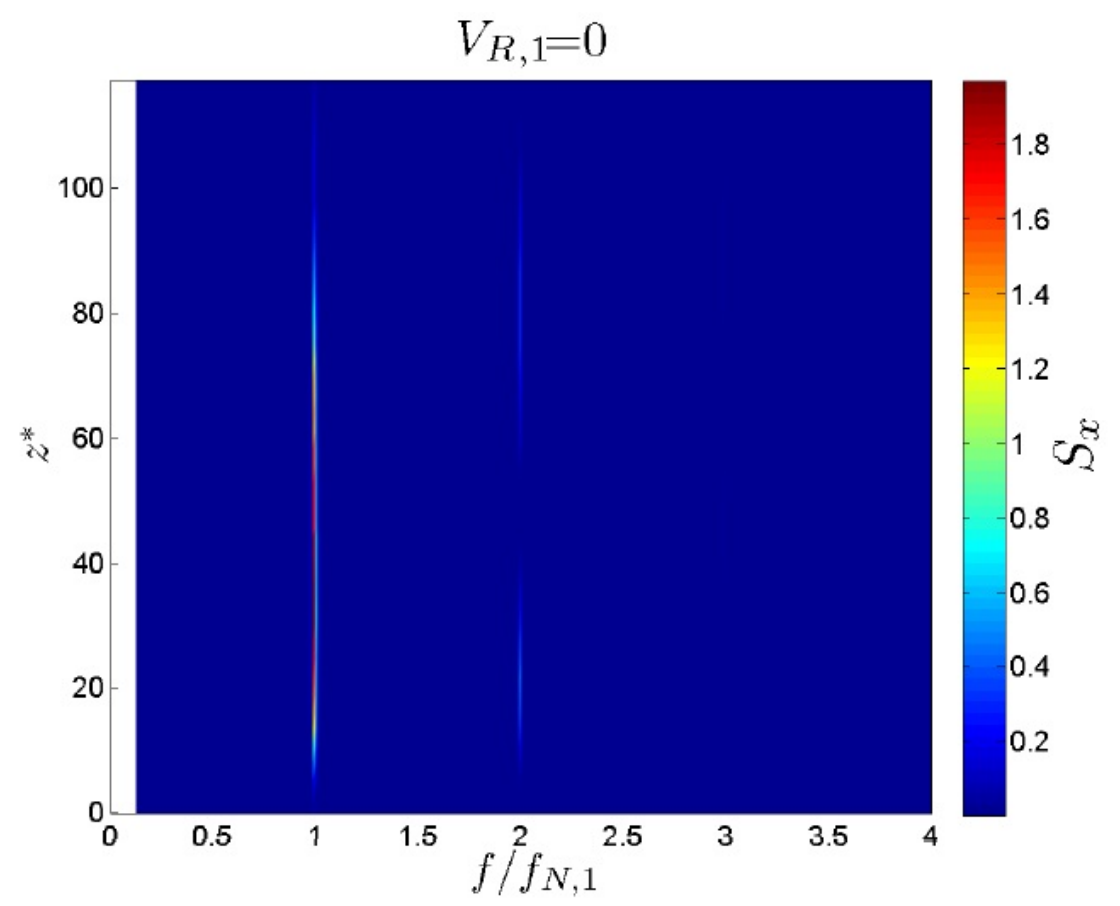

Source: Extracted from LIFE\&MO (2012d)

Following, it shall be considered the case in which $f_{t}: f_{N, 1}=3: 1$. The PSD of the in-line direction, see Figure 5.3, shows a monochromatic response, all energy within the third mode. The harmonic series should also appear, however, if the third harmonic is considered as fundamental, the next frequency would be twice its value, thus, the sixth mode. The sixth mode has little energy on it and it was not considered on the PSD plot. Is is also not observed any super-harmonic. 
Figure 5.3: PSD of imposed heave $-f_{t}: f_{N, 1}=3: 1$.

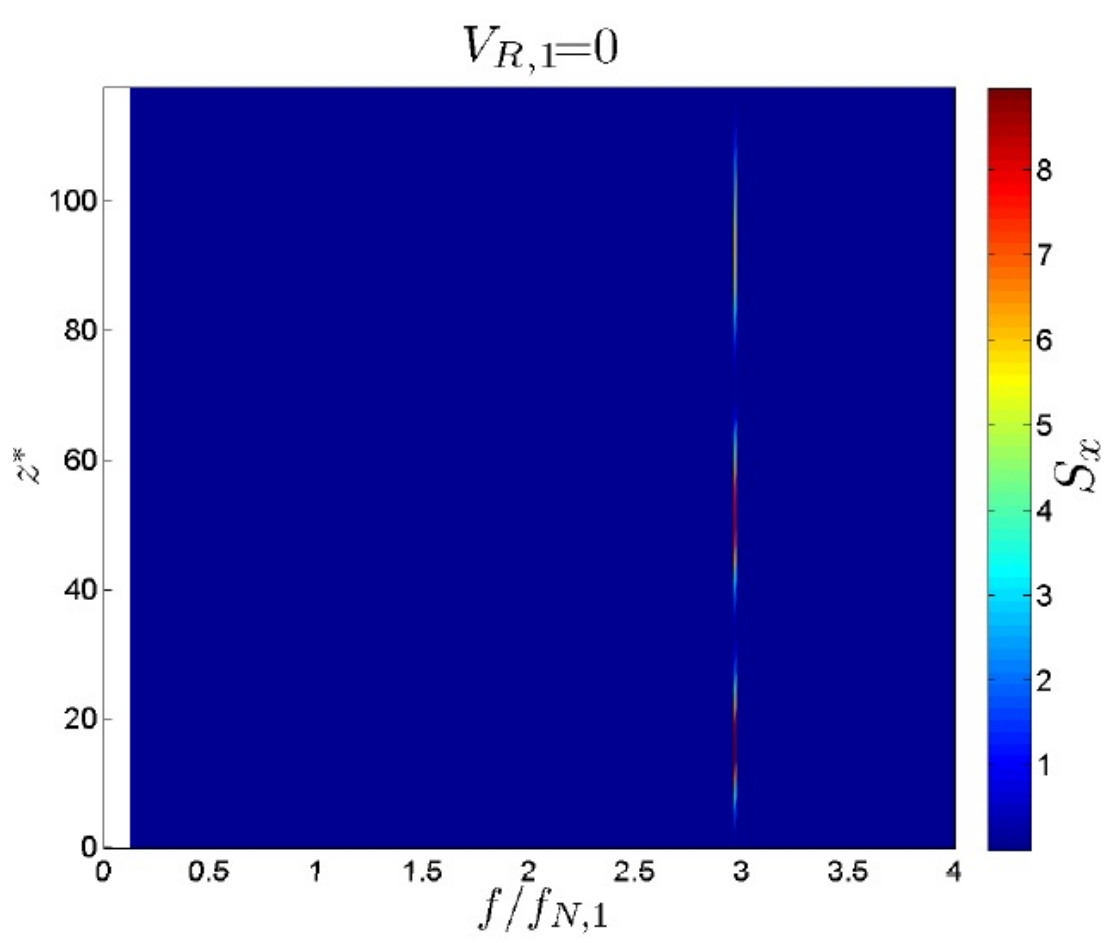

Source: Extracted from LIFE\&MO (2012d)

The case $f_{t}: f_{N, 1}=2: 1$ was left to be presented last on purpose, inasmuch as something really beautiful appears: Mathieu instability.

Figures 5.4a-d show much information about the model dynamics. The first phenomenon that calls attention is the "fork pattern" that can be seen on Figure 5.4a. The fork pattern indicates a Mathieu instability at the lower half of the model. Although the Mathieu instability occurs in the whole model, it appeared more visually in $5.4 \mathrm{a}$ at the lower half of the model.

Hereupon, whether it is considered the PSD of the crossflow motion plane, see Figure 5.4d, it might be seen a high energy peak in the second harmonic, but it is also possible to identify a weak energy peak of the fundamental mode. There is no coincidence and that might be seen regarding the PSD of the in-line direction, Figure $5.4 \mathrm{c}$, where the fundamental mode is the high energy peak, followed by the second harmonic. This shows that the modes are linked to each other, being able to transfer energy from one to the other. 
Figure 5.4: Scalograms and PSD spectra from imposed heave $-f_{t}: f_{N, 1}=2: 1$.

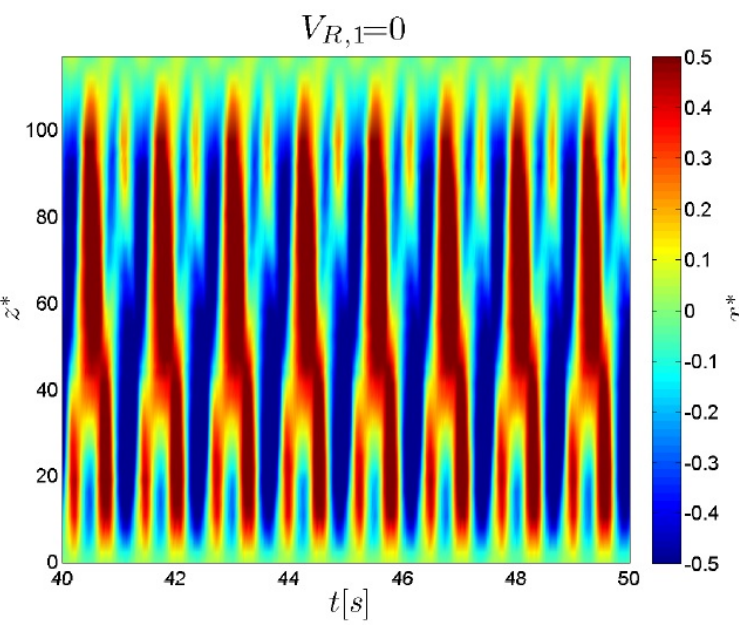

(a) Scalogram of $x^{\star}$ displacement.

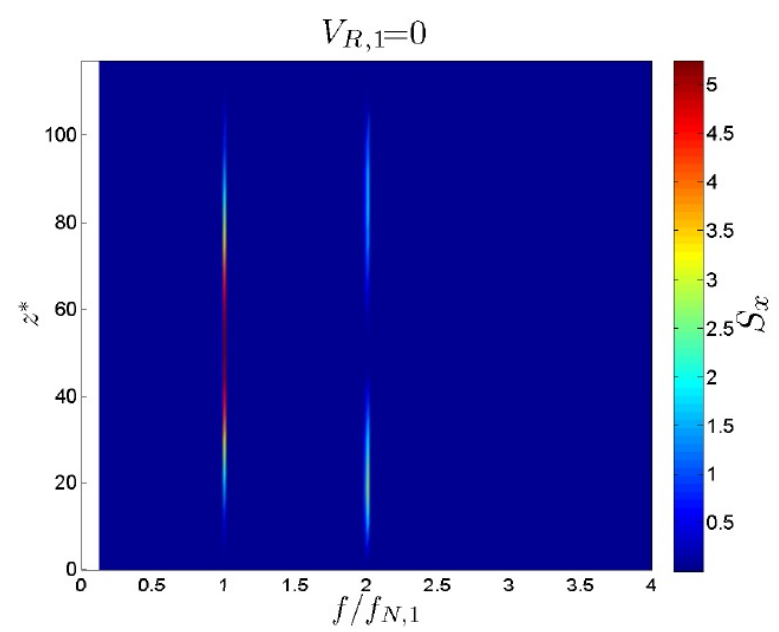

(c) PSD of $x^{\star}$.

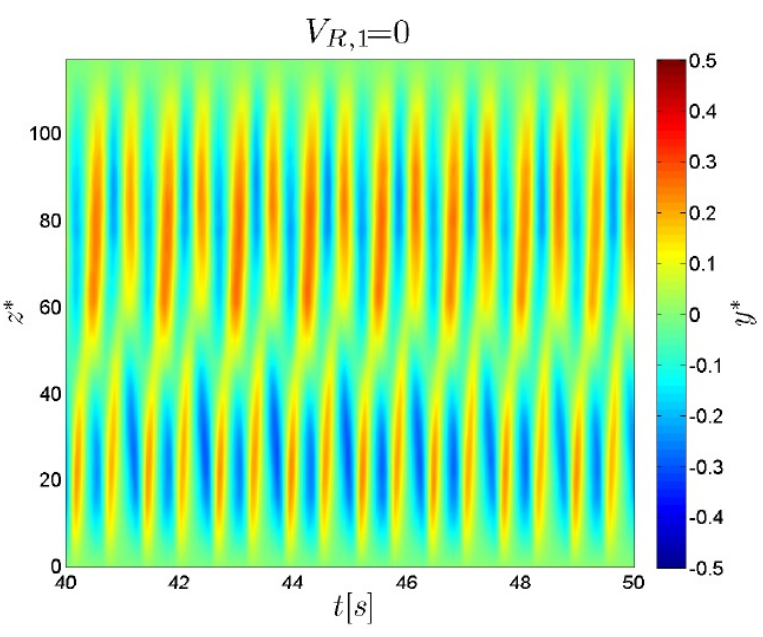

(b) Scalogram of $y^{\star}$ displacement.

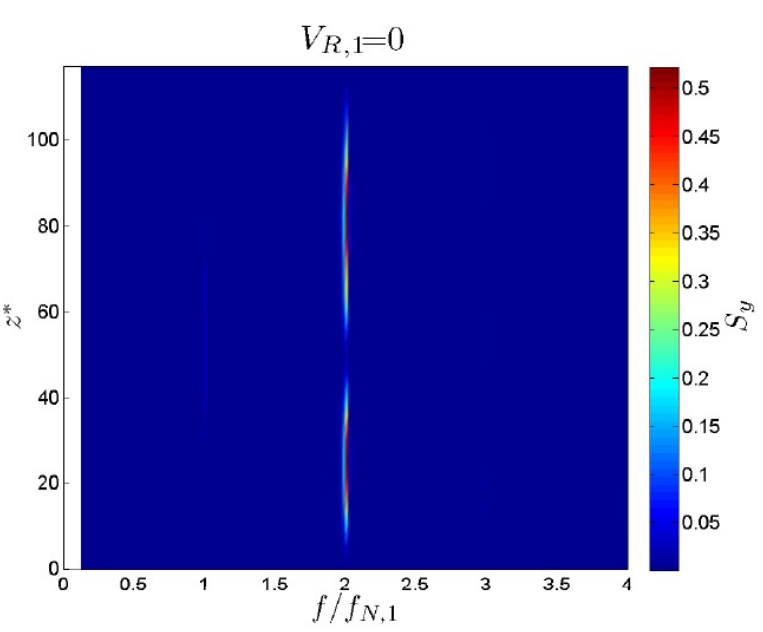

(d) PSD of $y^{\star}$.

Source: Extracted from LIFE\&MO (2012d)

Considering imposed heave, there was not any frequency above the third mode that could be seen in Figures 5.2, $5.3 \& 5.4 \mathrm{c}-\mathrm{d}$. If it is considered only phenomena related to heave, as shown, there is no necessity to use more than three modes to represent successfully the experimental elastica and there is a huge gain of analysis improvement, inasmuch as it is no longer necessary to analyse 43 temporal series in parallel, being sufficient only 3 up-to 4 modal temporal series to represent the same amount of dynamical information.

One final question remains about the modal representation: which modal eigenfunctions bases should be used in order to achieve a reliable result? According to what was discussed previously, the only requirement an eigenfunction base shall present is related to the Galerkin condition.

In Figure 5.5, it may be seen two different experimental data on the legend: 
1. Experimental (Exp.);

2. Experimental filtered (Exp. filt.).

The one called "experimental" refers to data without any pre-processing signal analysis, such as, filtering and windowing; the other data was processed using a band-pass filter, in order to give it a smoother aspect. In the end, the filtered data was not necessary, because the experimental data did not introduced much numerical error on the modal decomposition method.

Visually, the two modal bases are equivalent, but which one is more suitable? The answer is both are equivalently suitable. The argument to verify the equivalence comes from the Galerkin method. It is only necessary to a set of functions does not violate kinematical constrains in order to be a good base choice.

On the other hand, the sine base has shown to be a better modal decomposition choice for eigenvalues due to its simplicity. The Bessel-type eigenfunction requires the numerical solution of eq. 3.14, in order to determine the eigenvalue by the transcendental equation and, then, to obtain the modal shapes. On the other hand, the sine base does not require any of this, being much simpler to use numerically and analytically. 
Figure 5.5: Modal representation using three harmonics. Experimental data and Bessel-type eigenfunction base. VIV $-V_{R, 1}=6.00$.
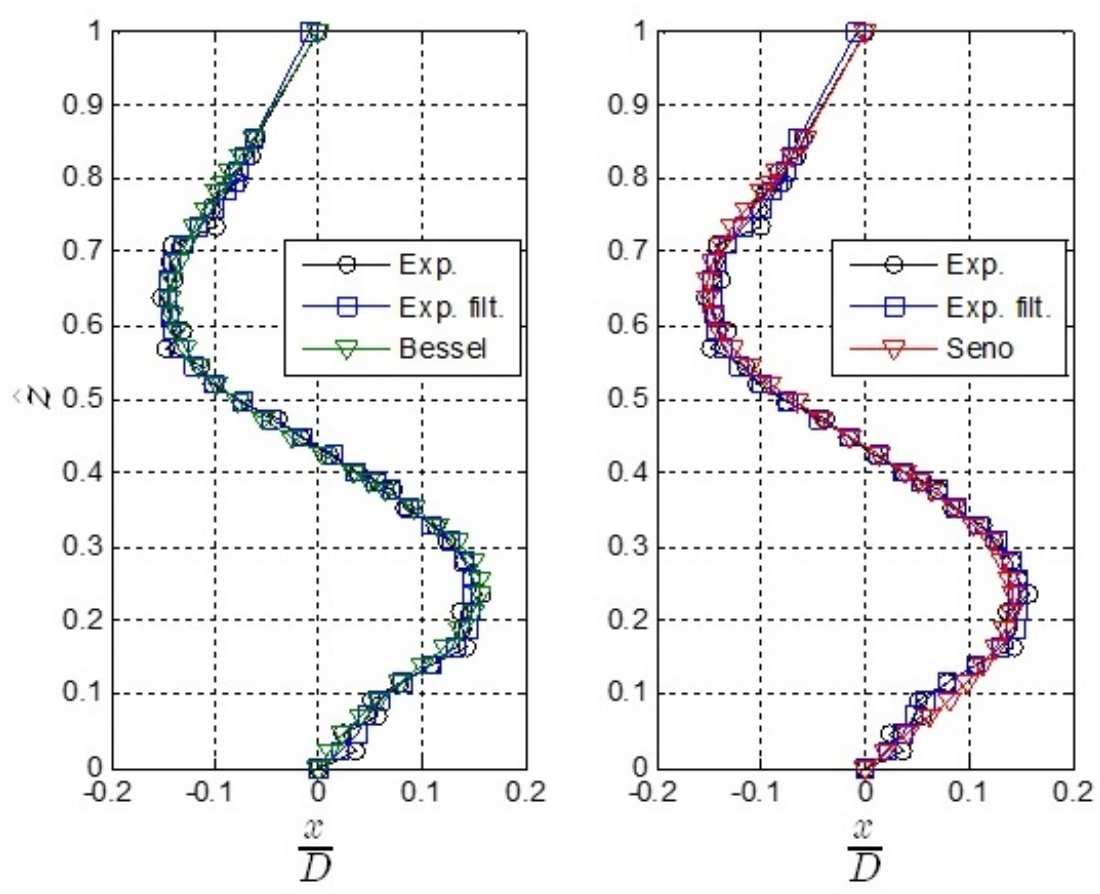

(a) $x^{\star}$ displacement.
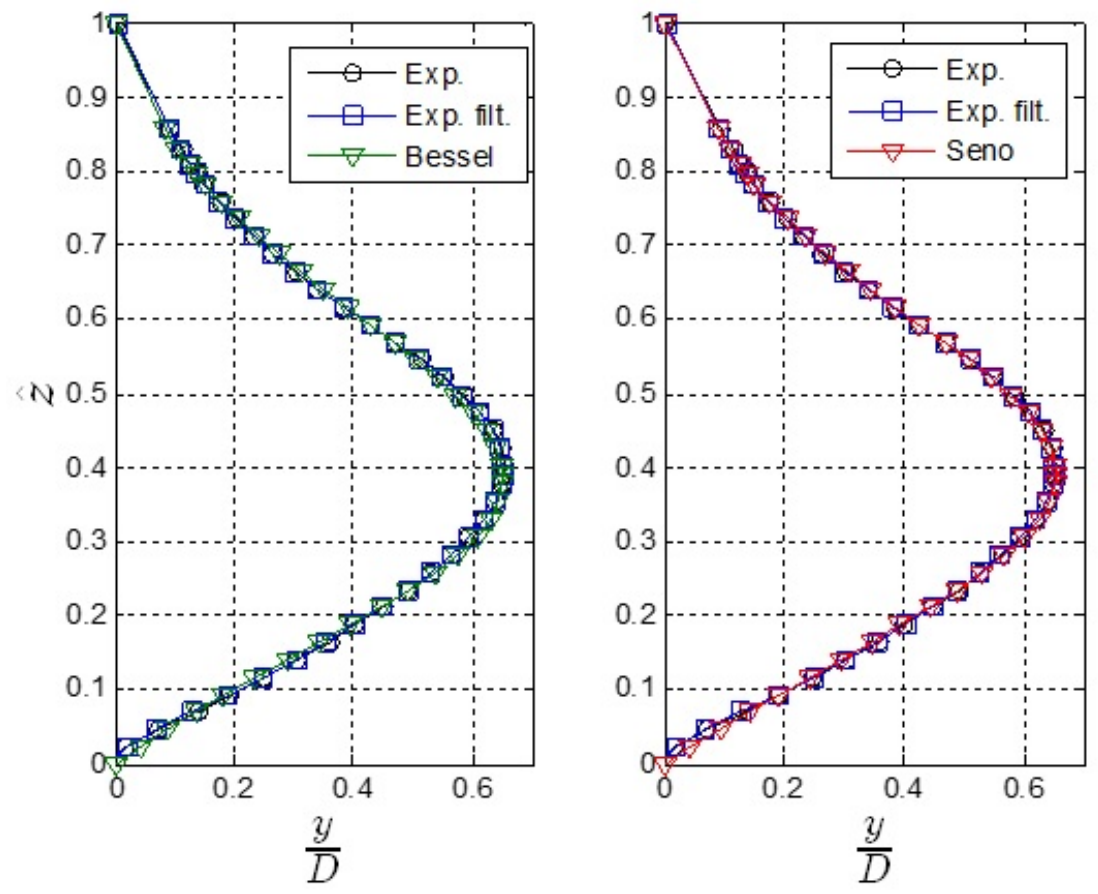

(b) $y^{\star}$ displacement.

The number of modes considered in which decomposition is related to how simpler the mode is. Sine modes are the simplest and they require a major number of harmonics in order to represent satisfactorily the elastica dynamics recomposition. More sophisticated modes as quasi-Bessel approach, see Mazzilli et al. (2014), have a closed-form solution 
that may be able to reduce the total number of harmonics. A non-linear mode would reduce the harmonic number even more. A small number of harmonics is desirable in order to obtain Reduced Order Models (ROMs) as compact as it is possible.

Hereupon, only the trigonometric base will be considered in modal analysis. It might be prudent testing the minimal number of harmonics, 3 up-to 4, suggested before is sufficient.

For the sake of imposed heave, it can be remarked 3 harmonics are sufficient to represent the $f_{t}: f_{N, 1}=2: 1$ case; see eq. 5.6. Despite the fact $f_{t}: f_{N, 1}=1: 1$ case is not shown, it was also well-represented by only 3 harmonics.

Figure 5.6: Modal representation using three harmonics. Experimental data and sine eigenfunction base. Heave $-f_{t}: f_{N, 1}=2: 1$.

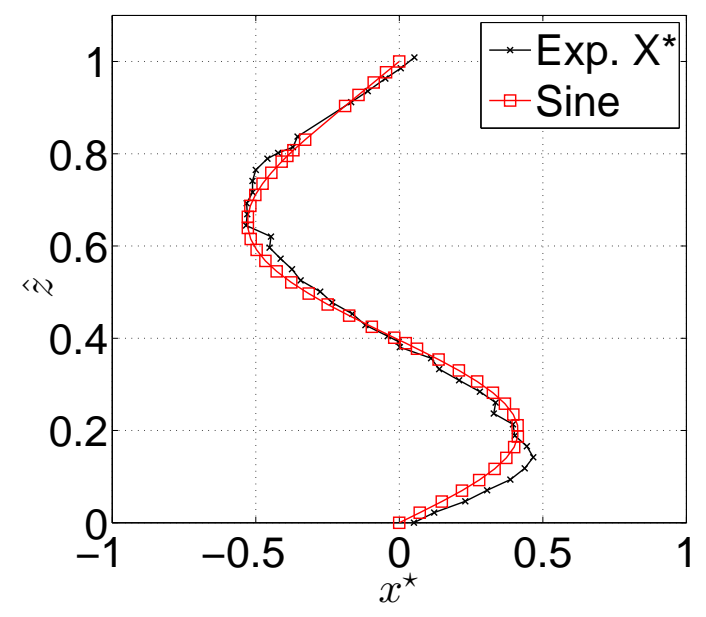

(a) Scalogram of $x^{\star}$ displacement.

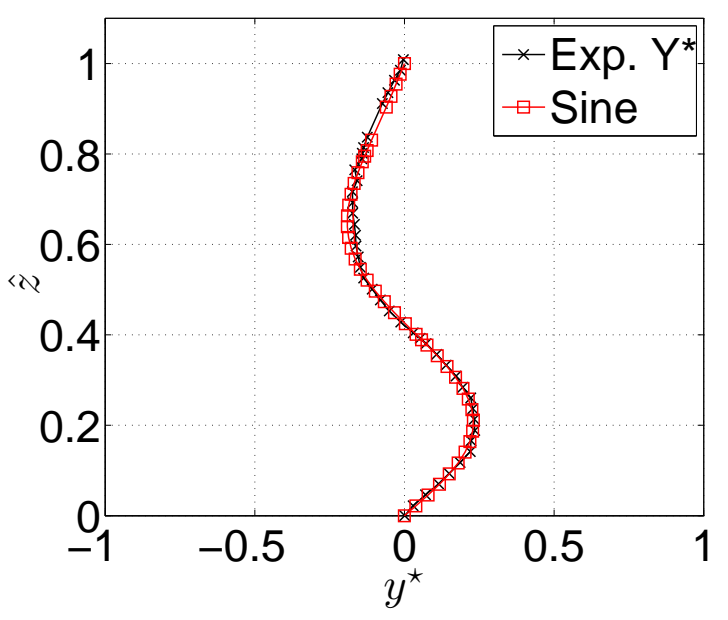

(b) Scalogram of $y^{\star}$ displacement.

Then again, when it is called attention to the $f_{t}: f_{N, 1}=3: 1$ case, something odd occurs. Whether the 3 harmonics representation is regarded, Figure 5.7, it will be seen that it is quite not as good as the others previously mentioned. Actually, in this particular case, the four harmonics modal decomposition is more suitable, reaching a very satisfying result; see Figure 5.8 . 
Figure 5.7: Modal representation using three harmonics. Experimental data and sine eigenfunction base. Heave $-f_{t}: f_{N, 1}=3: 1$.

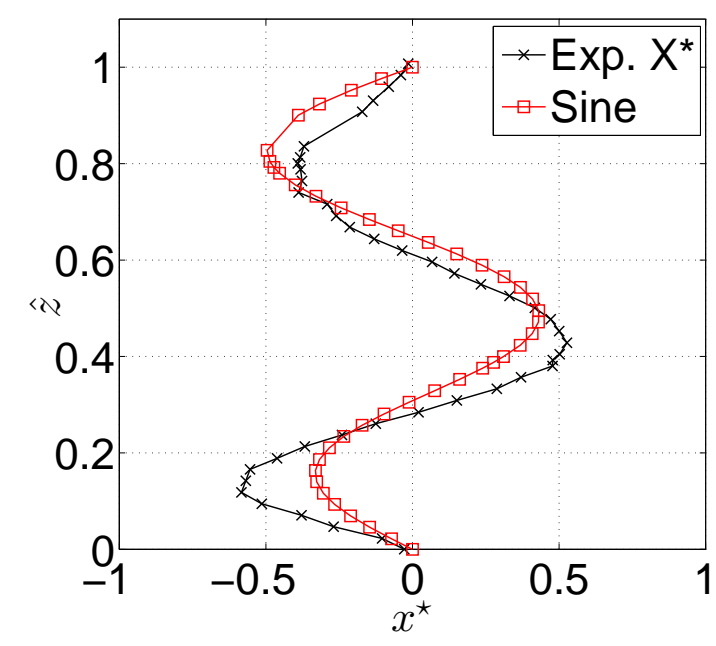

(a) Scalogram of $x^{\star}$ displacement.

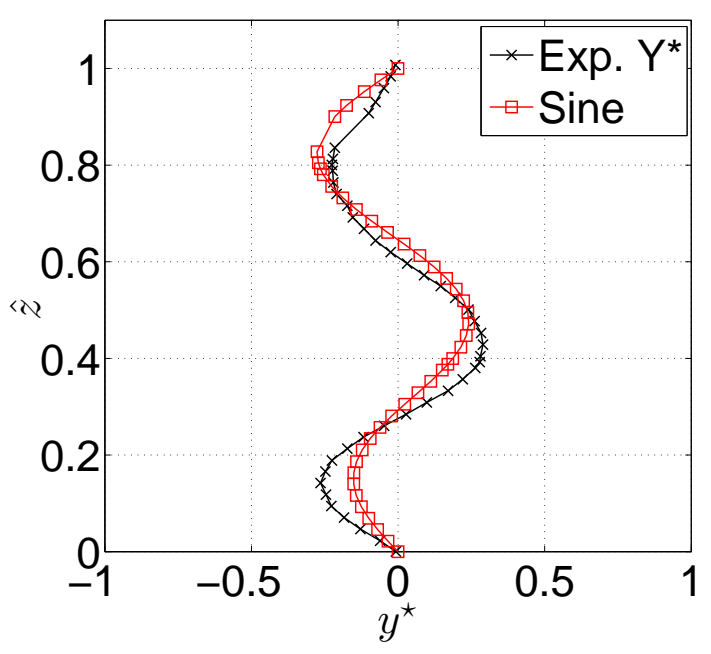

(b) Scalogram of $y^{\star}$ displacement.

Figure 5.8: Modal representation using four harmonics. Experimental data and sine eigenfunction base. Heave $-f_{t}: f_{N, 1}=3: 1$.

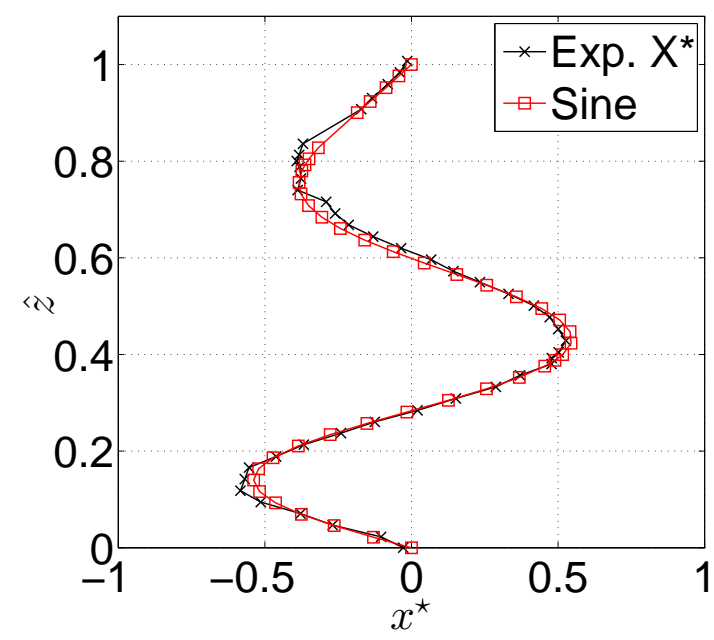

(a) Scalogram of $x^{\star}$ displacement.

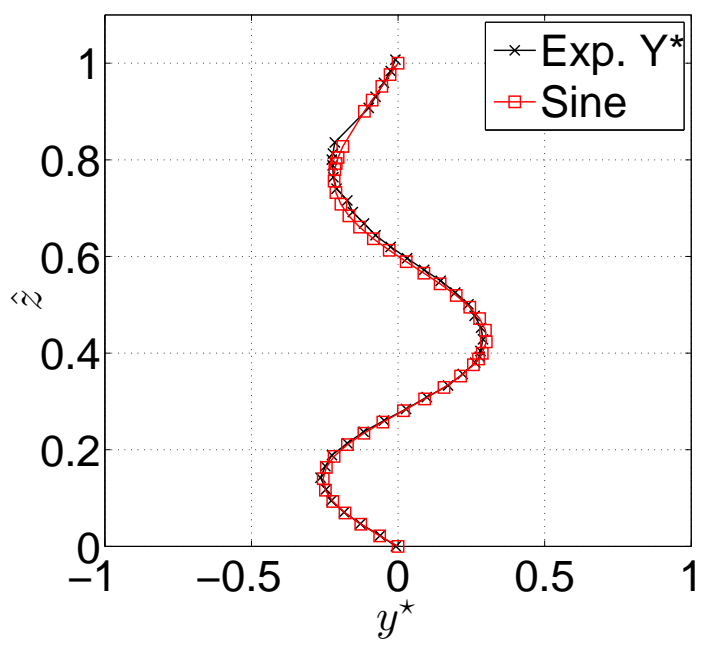

(b) Scalogram of $y^{\star}$ displacement.

Using Sine modes, four harmonics have shown to be a better choice. If it was used, e.g. quasi-Bessel modes, the total amount of harmonic could be less than four. 
Finally, the modal decomposition used might be summed-up as:

i. Sine modal eigenfunctions;

ii. Number of harmonics to be used: 3 up-to 4 .

\subsection{Free-Decay Test in air}

Using a free-decay test in air, it is possible to isolate the effect caused from structural damping, since it is possible to neglect drag force due to the air. The modal structural damping coefficients will be the first result that follows. Then, it is evaluated the modal added mass coefficient for the experiment in water, relating it with the experiment in air.

There are three different experimental tests that consist the free decay in air, called: Test 4; Test 5; and Test 6 . The difference among them are simply the position in the model spanwise in which the initial impulse was given. Figure 5.9 shows schematically the impulse position in each test.

Figure 5.9: Free-Decay Test - Initial impulse position along spanwise. Impulse A - Test 4; Impulse B Test 5; and Impulse C - Test 6

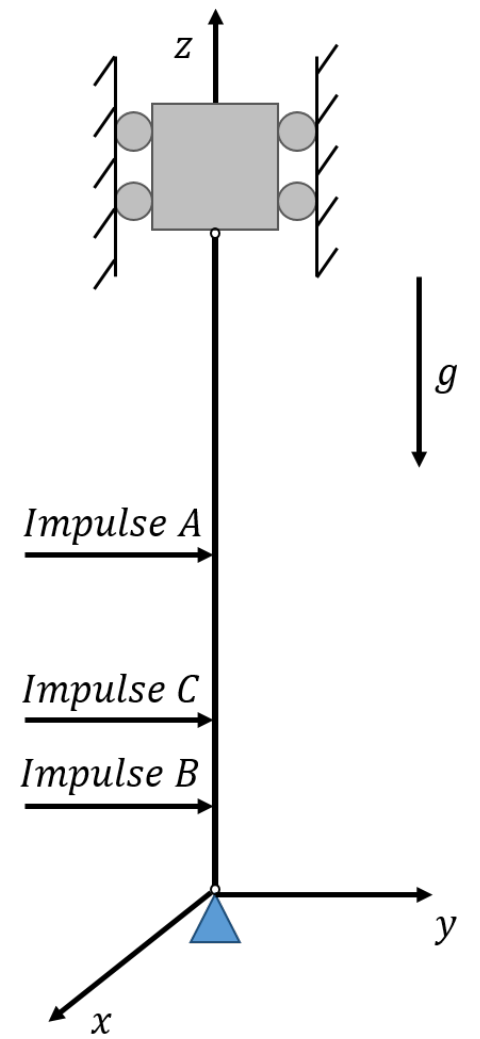

\subsubsection{Modal Structural Damping Coefficient}

An example of a free-decay time series may be regarded in Fig.5.10. The target chosen is placed in the model middle spanwise. From the time series of each target on the model, 
the modal decomposition is evaluated using trigonometrical functions, obtaining modal time series; see Fig.5.15.

Figure 5.10: Free-Decay Test - Time Series of the Target 25 .
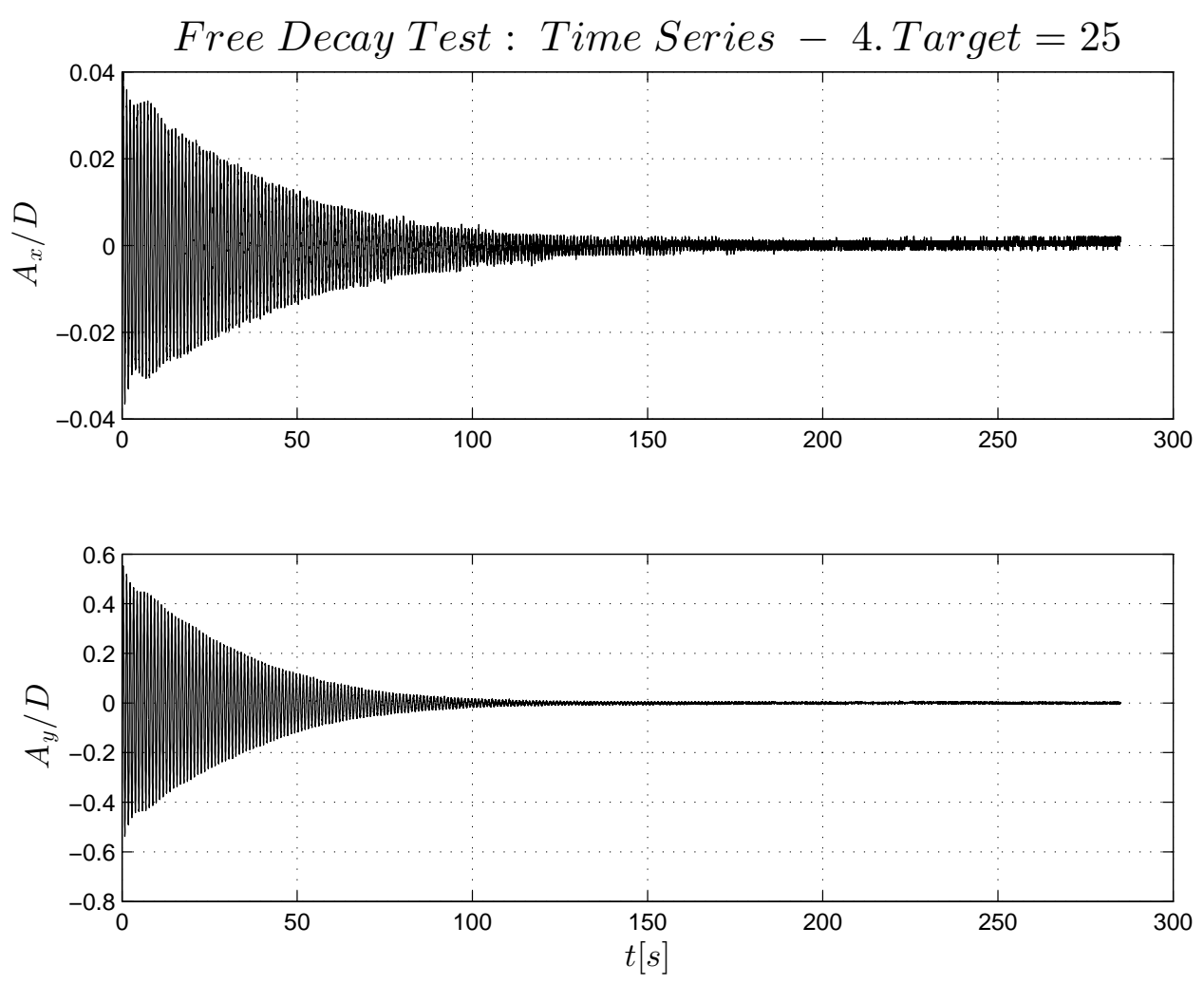

Figure 5.11 presents the scalogram of each experimental test. There is a predominance of the first mode in the free decay test movement in air. Such behavior is confirmed analyzing the amplitude spectra ${ }^{1}$ of all tests, see Figure 5.12-5.14, in which the power is evaluated in $d B$, showing that the great majority of energy is confined in the first mode, being necessary to decay almost two decades to reach the energy levels of second and third modes.

\footnotetext{
${ }^{1}$ The amplitude spectrum was obtained using the Hilbert-Huang transform; see Gonçalves et al. (2012) for more details of the technique.
} 
Figure 5.11: Free-Decay Test - Amplitude scalogram $\left(y^{\star}\right)$.

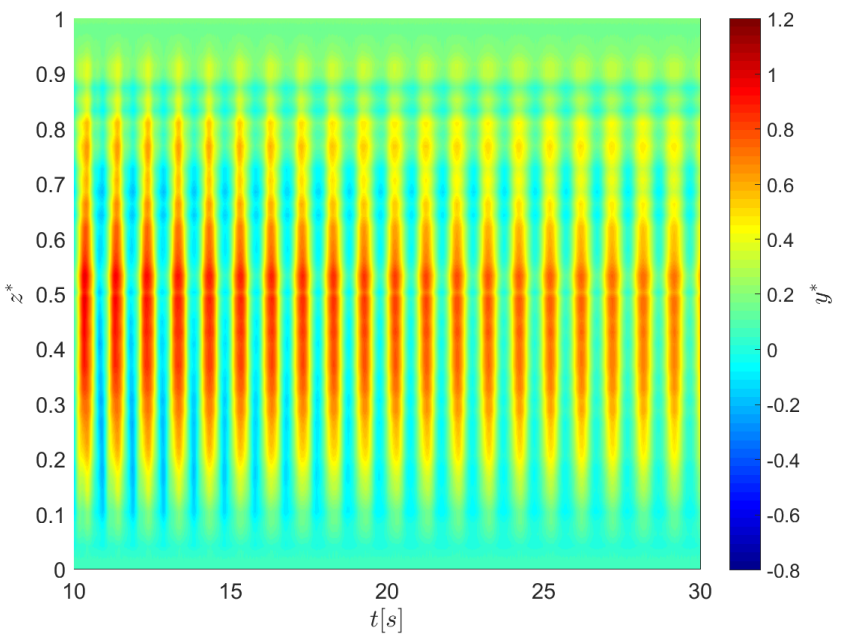

(a) Test 4

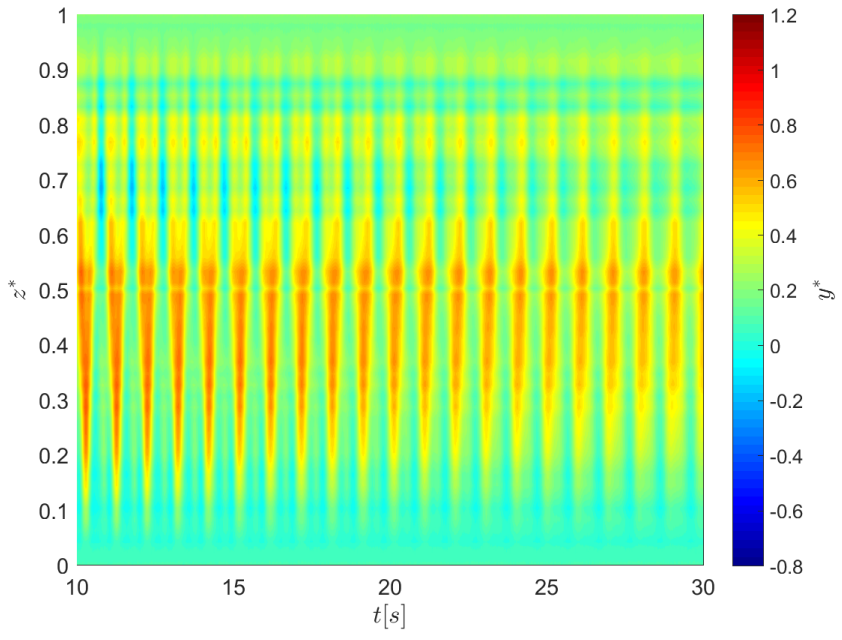

(b) Test 5

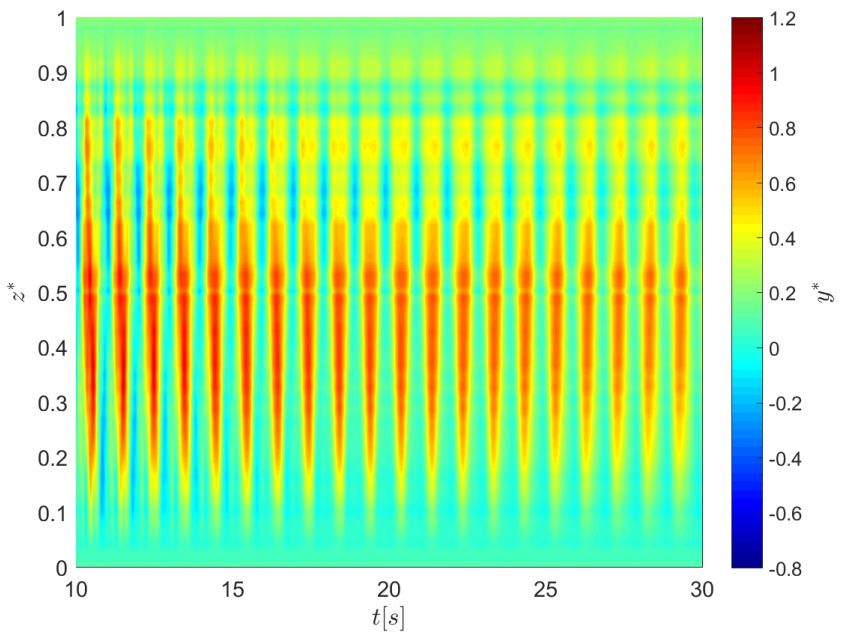

(c) Test 6 
Figure 5.12: Free-Decay - Amplitude Spectrum Density along spanwise: Test 4.

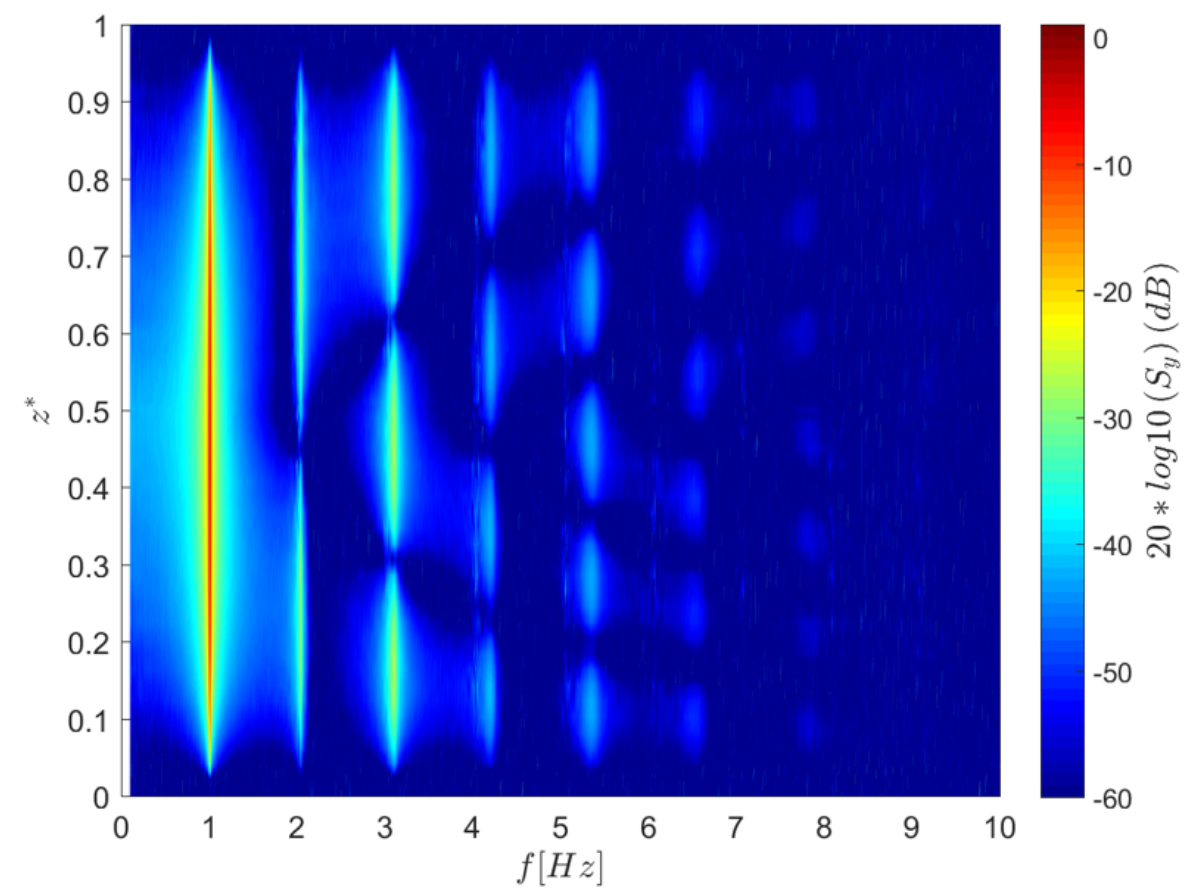

Figure 5.13: Free-Decay - Amplitude Spectrum Density along spanwise: Test 5

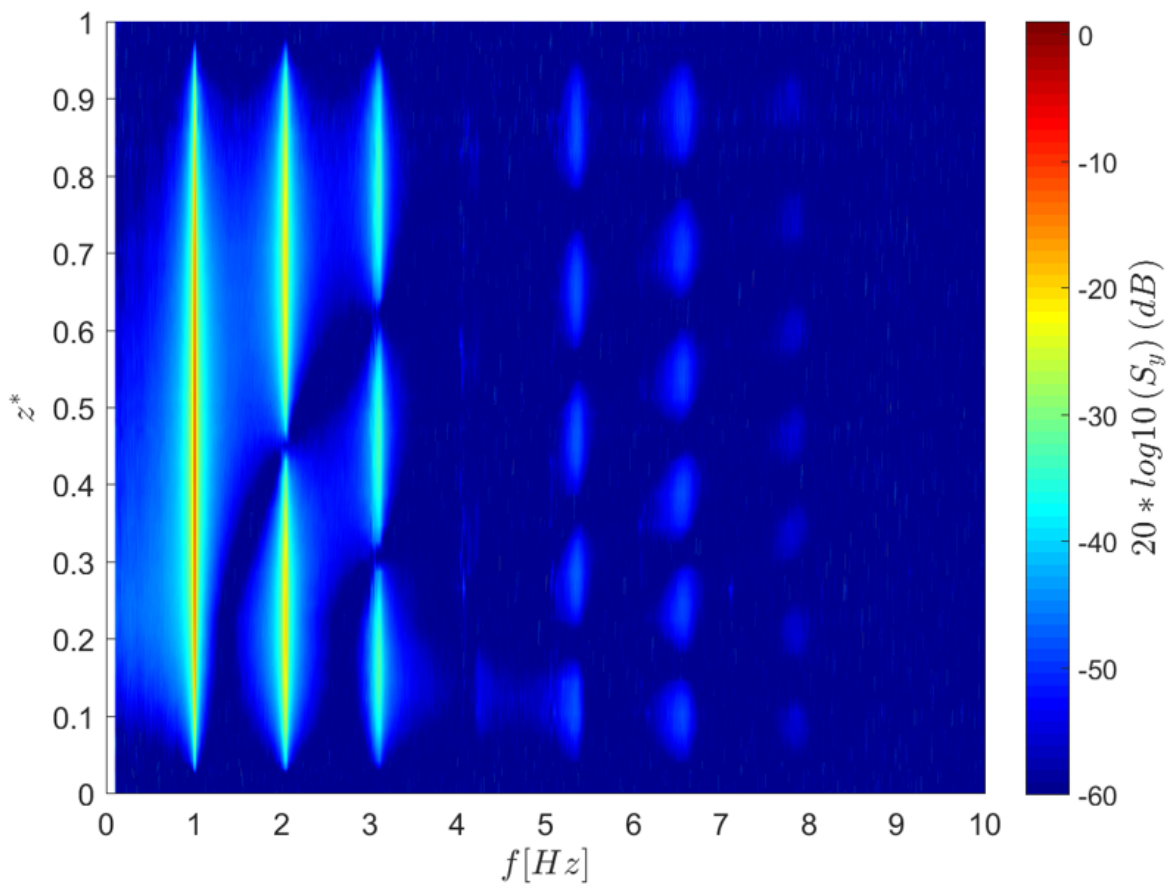


Figure 5.14: Free-Decay - Amplitude Spectrum Density along spanwise: Test 6

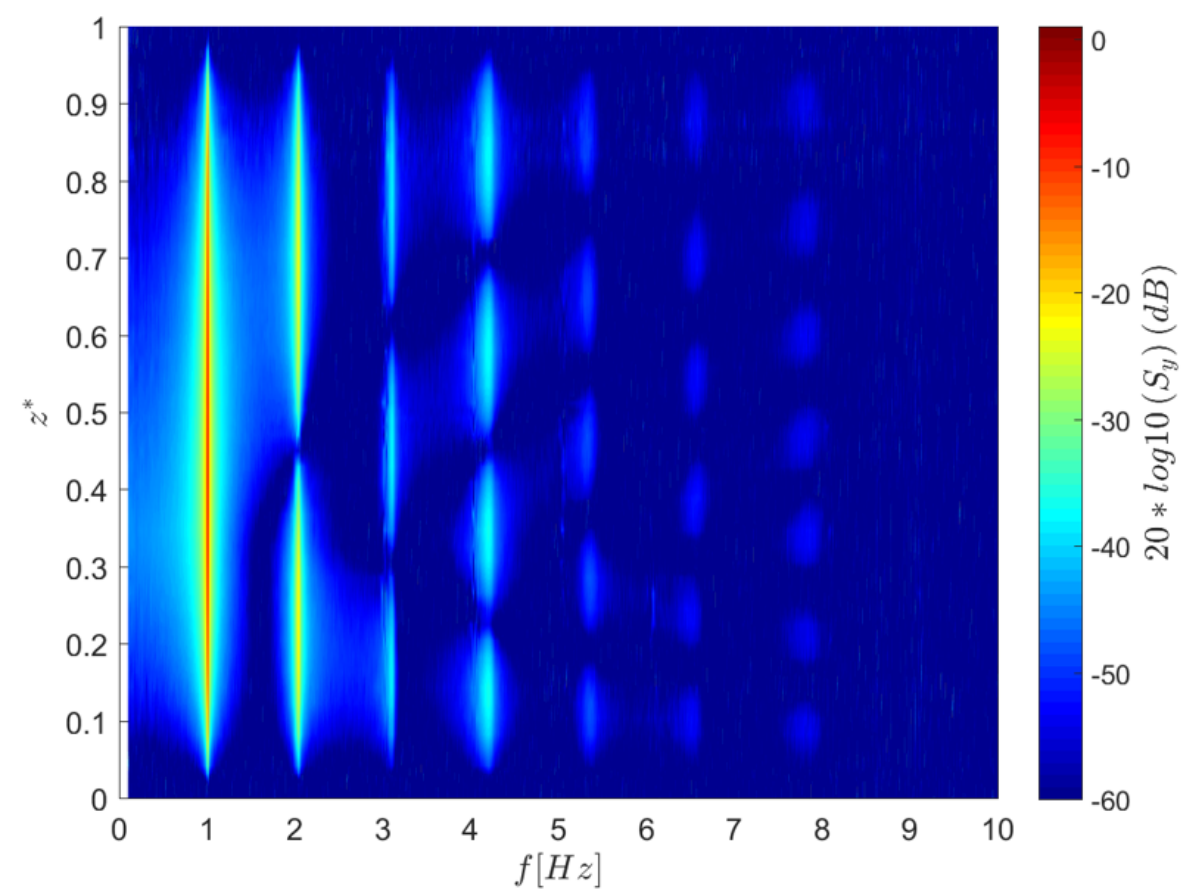

Figure 5.15: Free-Decay Test - Modal Amplitude Time Series.
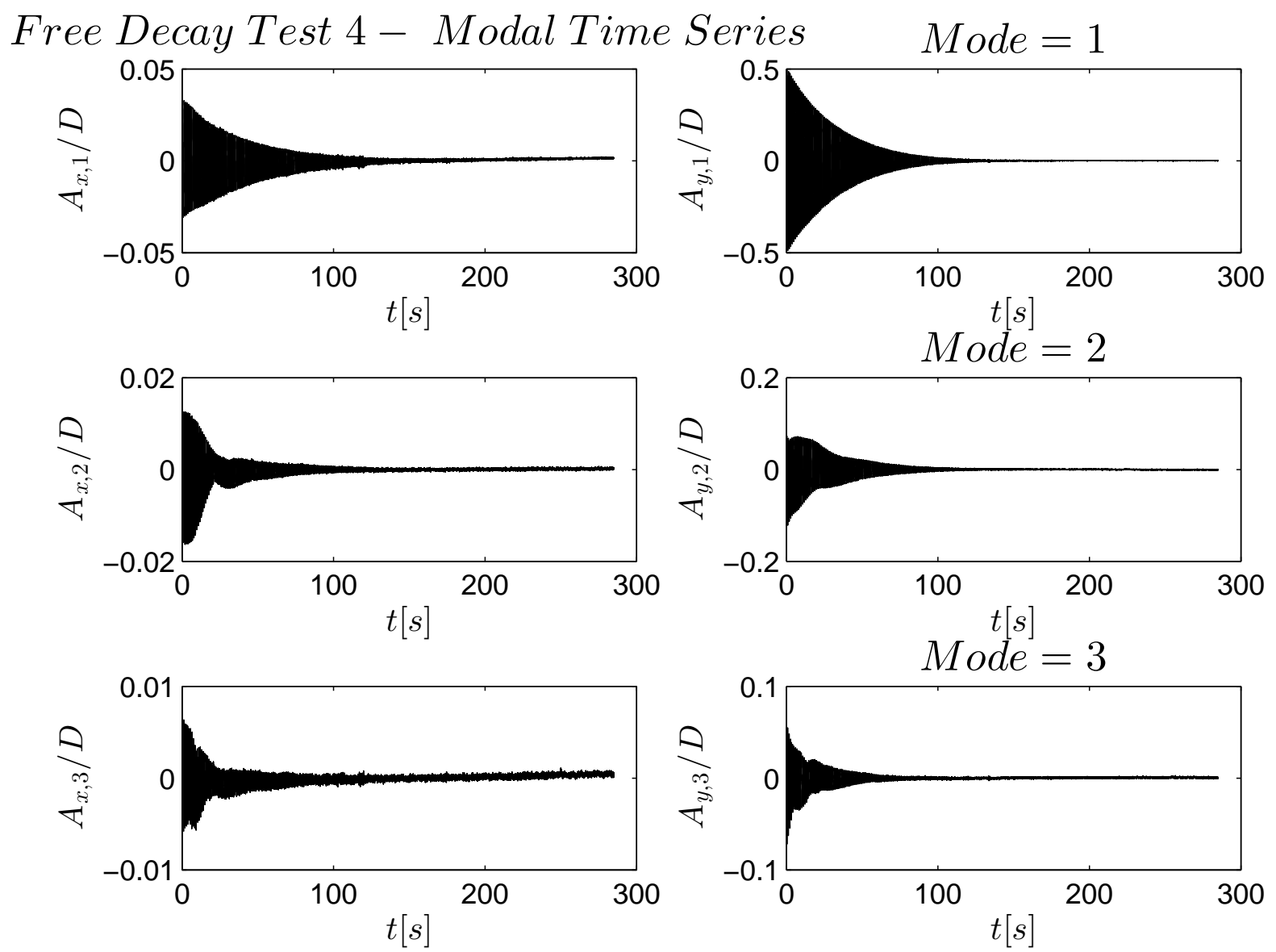
Figure 5.15 shows that the first mode behaves very smoothly, but the second and third modes show evidences of being polichromatic. Besides, the movement is larger in the $Y$ direction, as the free-decay impulse was given in that direction.

Now considering only de $Y$ direction movement and looking more closely to the modal series, Figures 5.16-5.27 show that the chosen modal decomposition using a trigonometric base produces a modal amplitude series that is not monochromatic. The amplitude spectrogram shown in Figures 5.16-5.27 were obtained by creating a classic FFT spectrum of each one and, by windowing samples, one-by-one. In order to evaluate the FFT in each sample, a Kaiser window was used, so the spectra information is centered in the middle of the sample. The energy within each frequency in the amplitude spectrum is given in $d B / H z$.

Using Test 4 as an example, Figures 5.17, 5.19 and 5.21 bring attention to some interesting aspects of each modal amplitude series. The first mode is essentially monochromatic and it is a general decaying response, as aforementioned when the Figure5.15 was presented. The next modes do not have the same fortune, needing more attention.

The second mode has a composition of frequencies in which the dominant response has the frequency of the first mode. The damping effect of each mode appears to increase with the number mode, inasmuch as the higher modes seems to decay faster than the first. In order to estimate the damping coefficient of any mode, the initial signal interval is necessary, but that is the region where the mixture of harmonics causes a unsuitable signal behavior to be analyzed.

A simple band-pass filter was applied in the signal FFT spectrum, trying to reach a modal series suitable for the decaying analysis. The second mode is filtered as shown in Figure 5.19 and the filtered temporal series is simply evaluated with the inverse FFT. The result obtained is not close to the original signal and it leaded to a damping coefficient that needs to be analyzed together with the damping coefficient obtained with the other tests.

When the same procedure is used with the third mode, see Figure 5.21, the reconstructed signal is very close to the original, providing a damping coefficient more suitable.

Table 5.1 presents a summary of all damping coefficients and natural frequencies estimates using data from Figures 5.18, 5.20, 5.22, 5.24-5.26, 5.28-5.30. Adopting a visual criteria when comparing the reconstructed filtered signal in time domain, the more suitable results considered to be valid are emphasized in Table 5.1 and the mean values of modal structural linear damping coefficients and natural frequencies are given in Table 5.2. 
Figure 5.16: Free-Decay Test $4-$ Amplitude spectrogram $\left(y^{\star}\right)$.

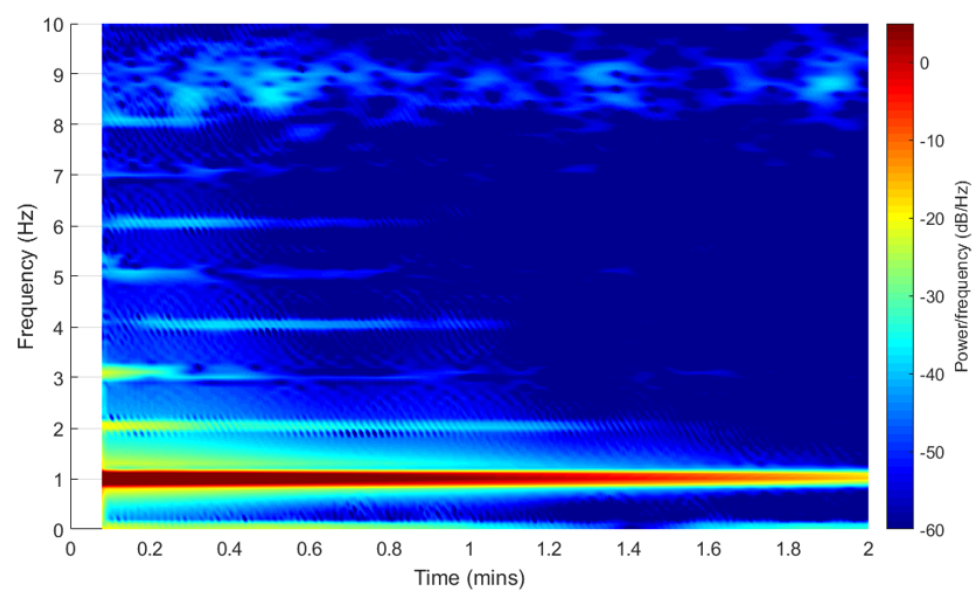

(a) First mode

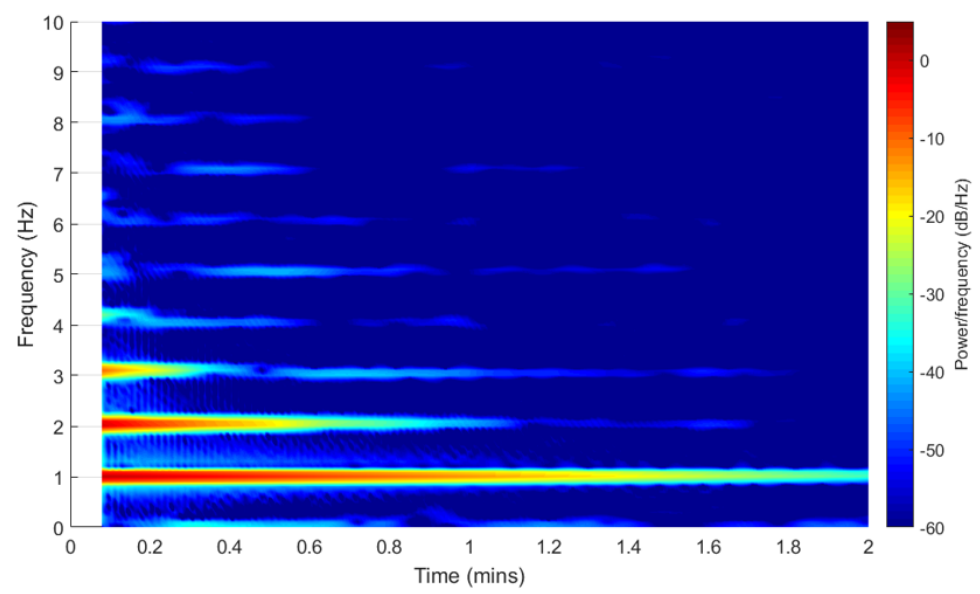

(b) Second mode

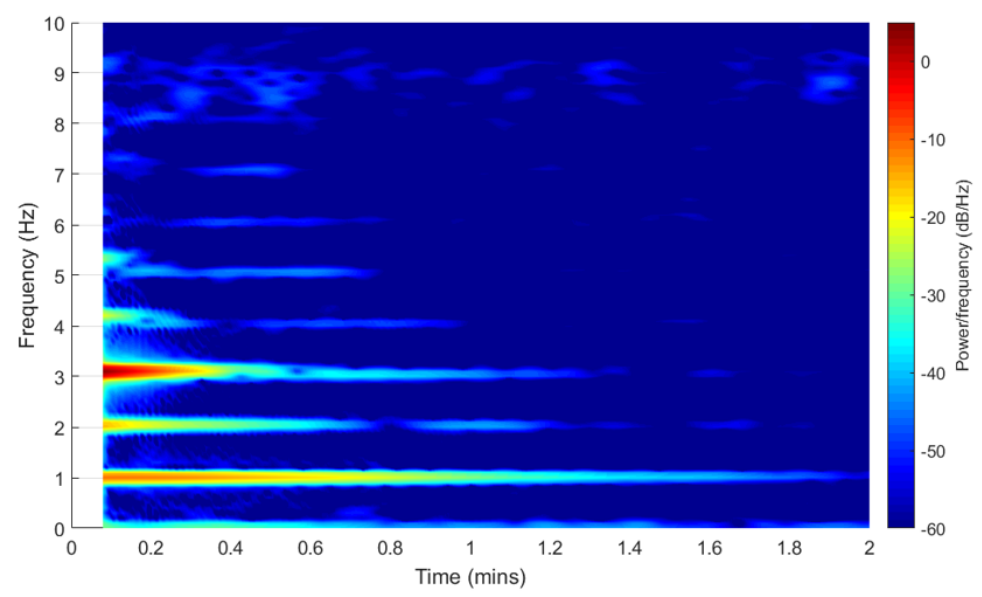

(c) Third mode 
Figure 5.17: Free-Decay Test - Modal Amplitude Time Series.
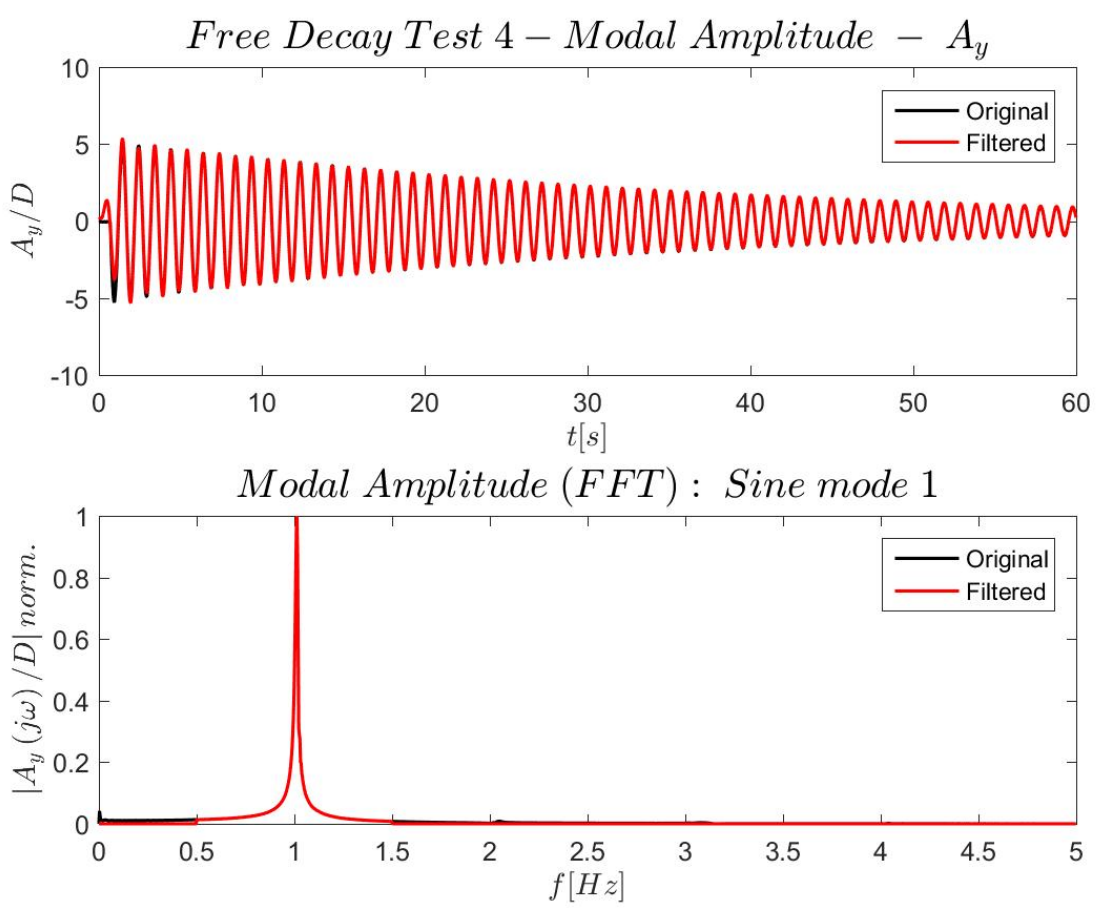

Figure 5.18: Free-Decay Test 4 - Modal linear damping: Mode 1.
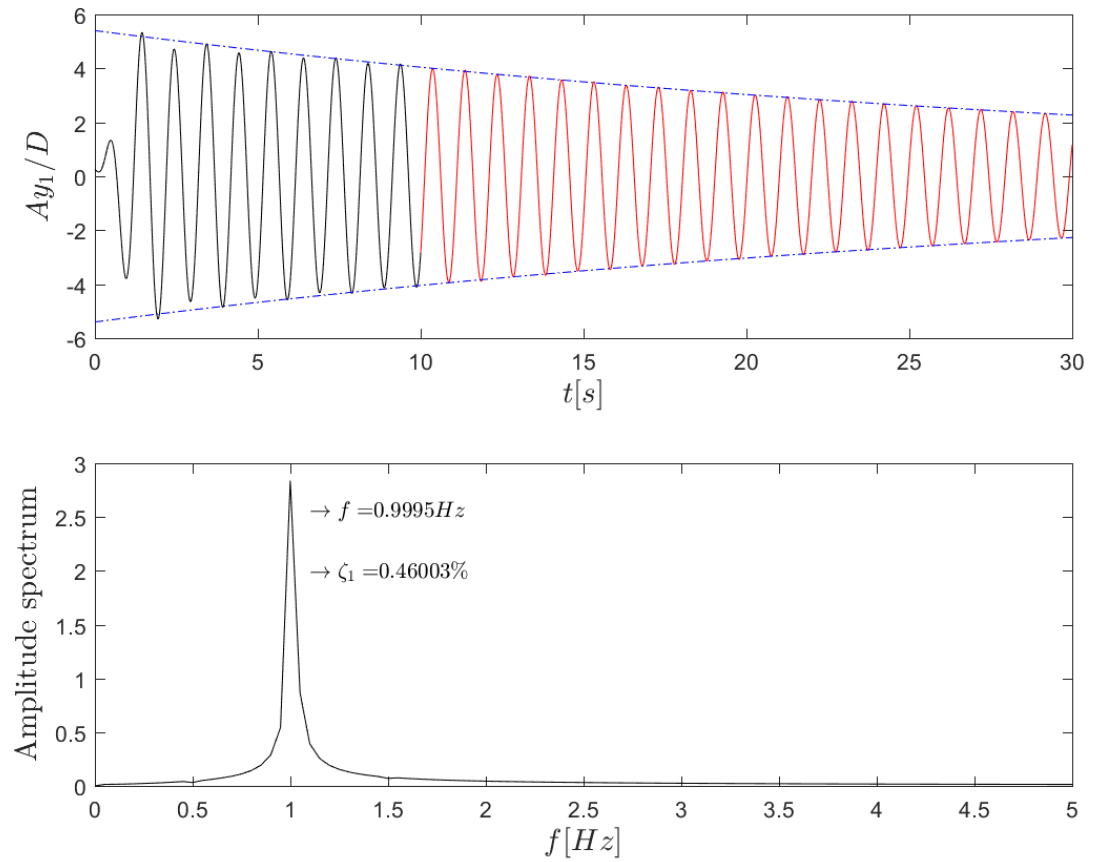
Figure 5.19: Free-Decay Test - Modal Amplitude Time Series.
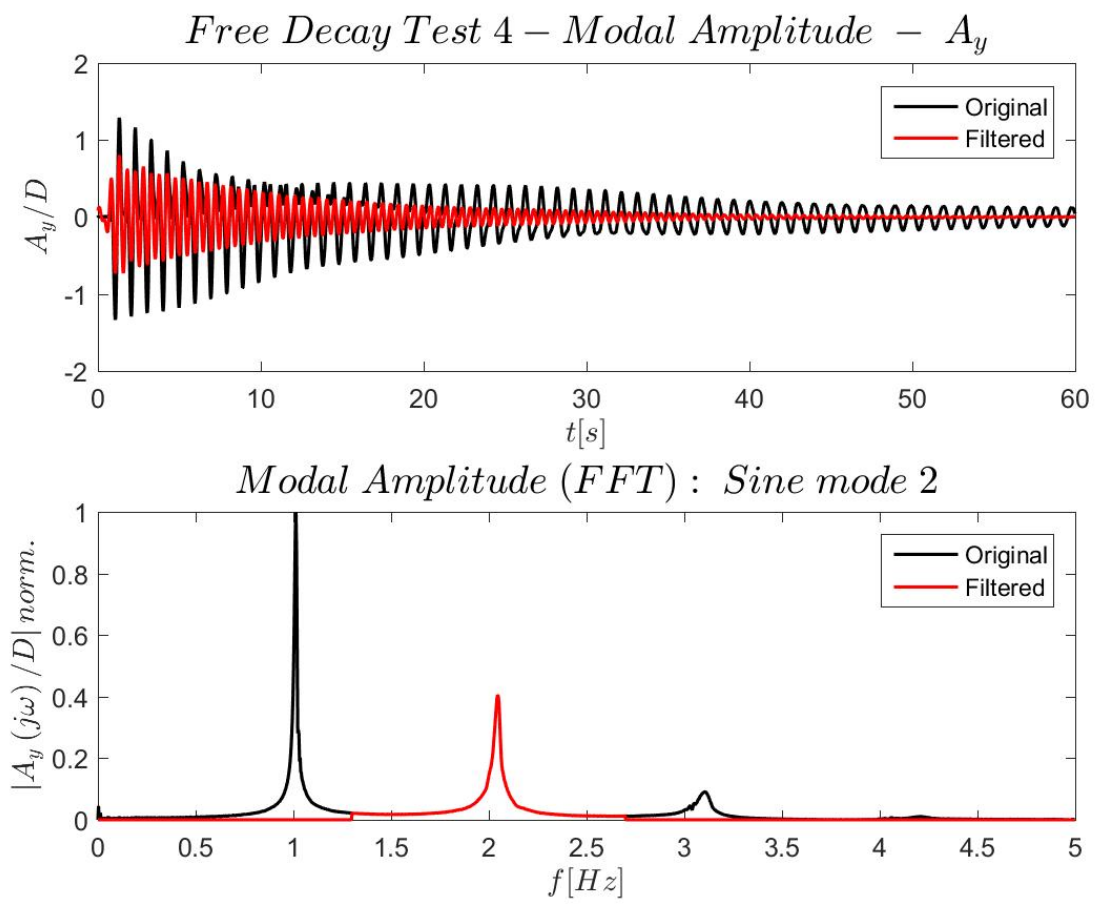

Figure 5.20: Free-Decay Test 4 - Modal linear damping: Mode 2.
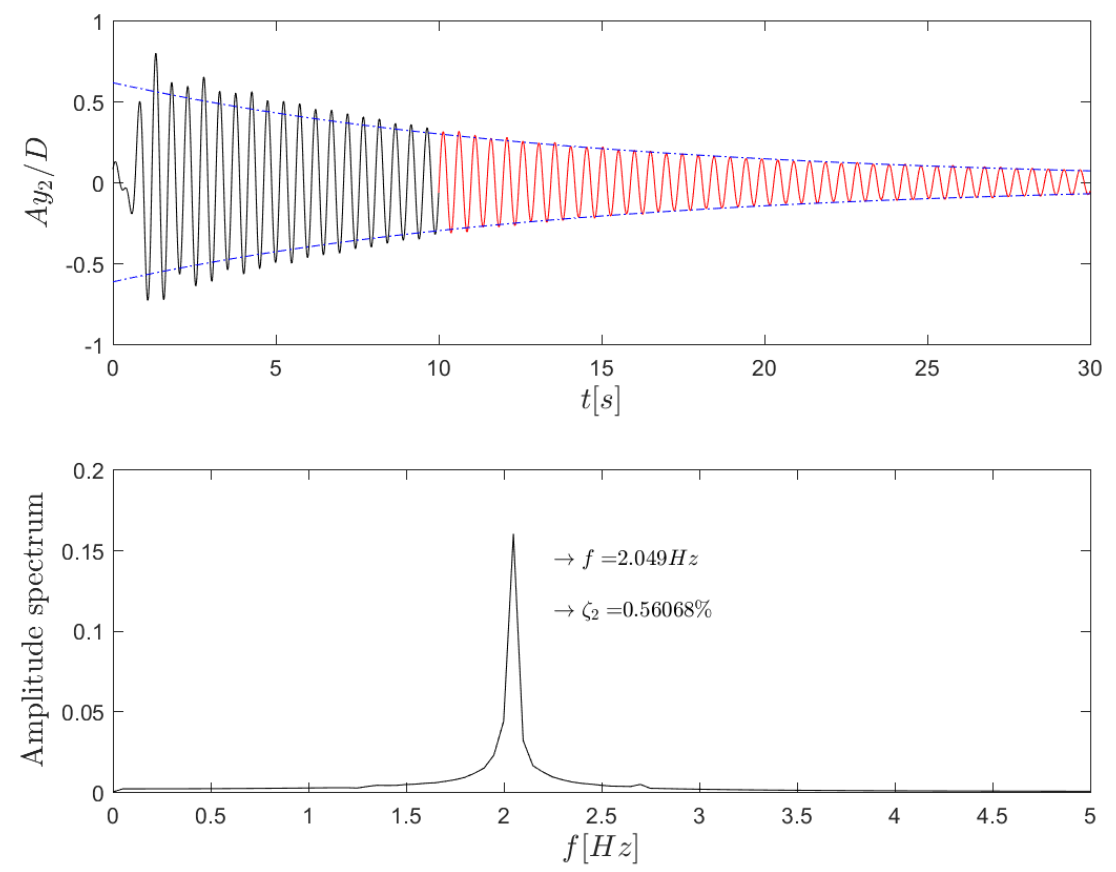
Figure 5.21: Free-Decay Test - Modal Amplitude Time Series.
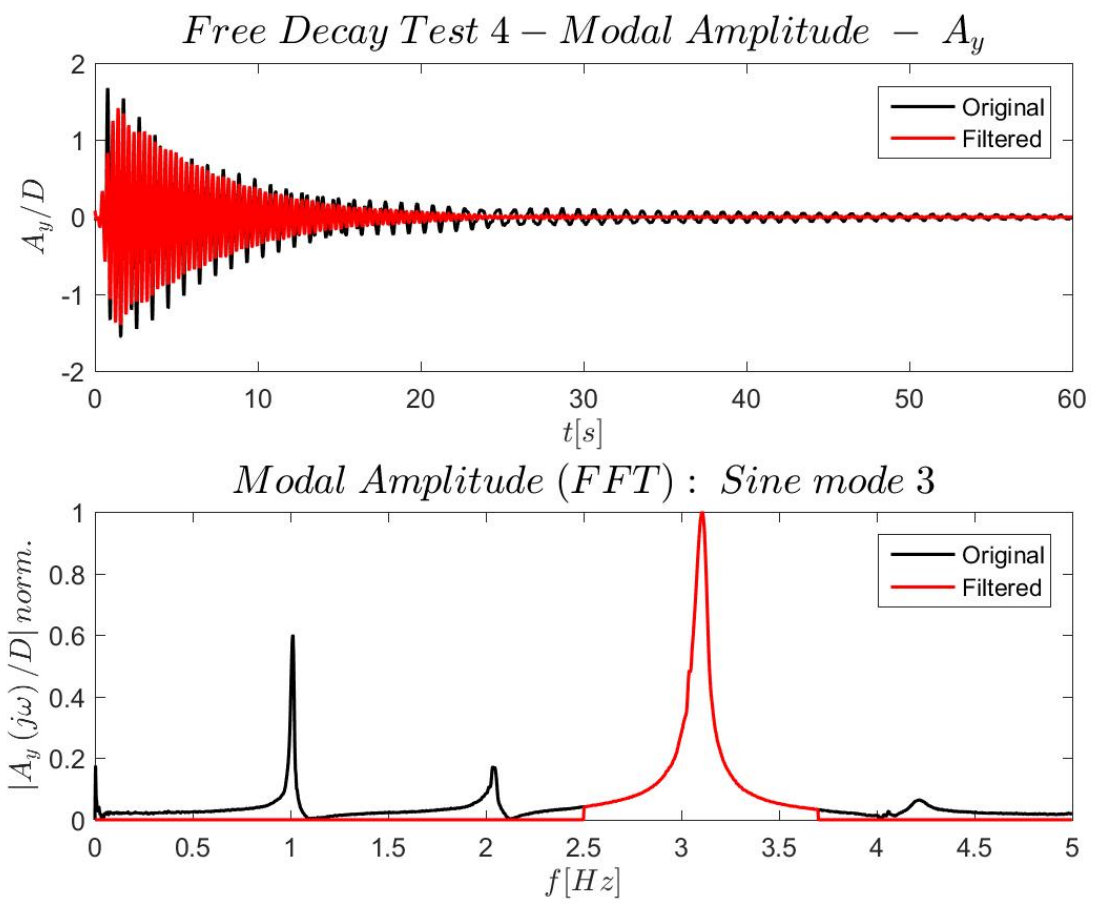

Figure 5.22: Free-Decay Test 4 - Modal linear damping: Mode 3.
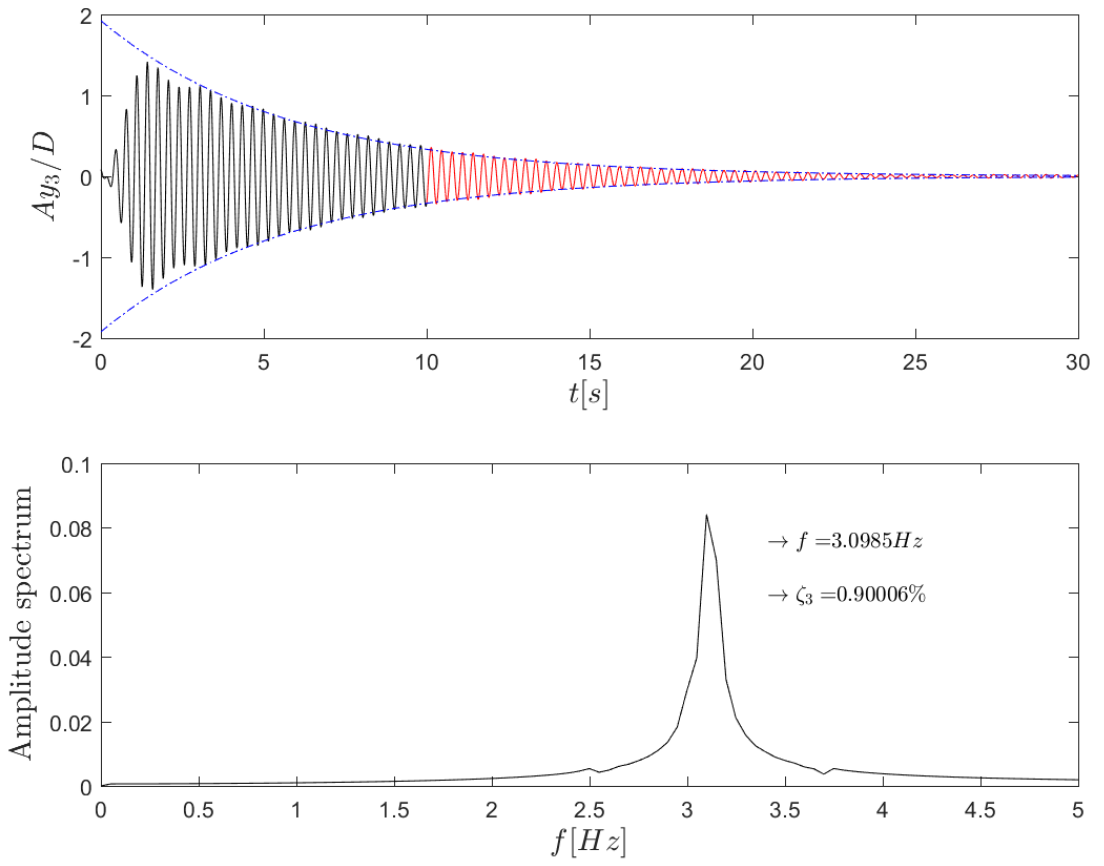
Figure 5.23: Free-Decay Test 5 - Amplitude spectrogram $\left(y^{\star}\right)$.

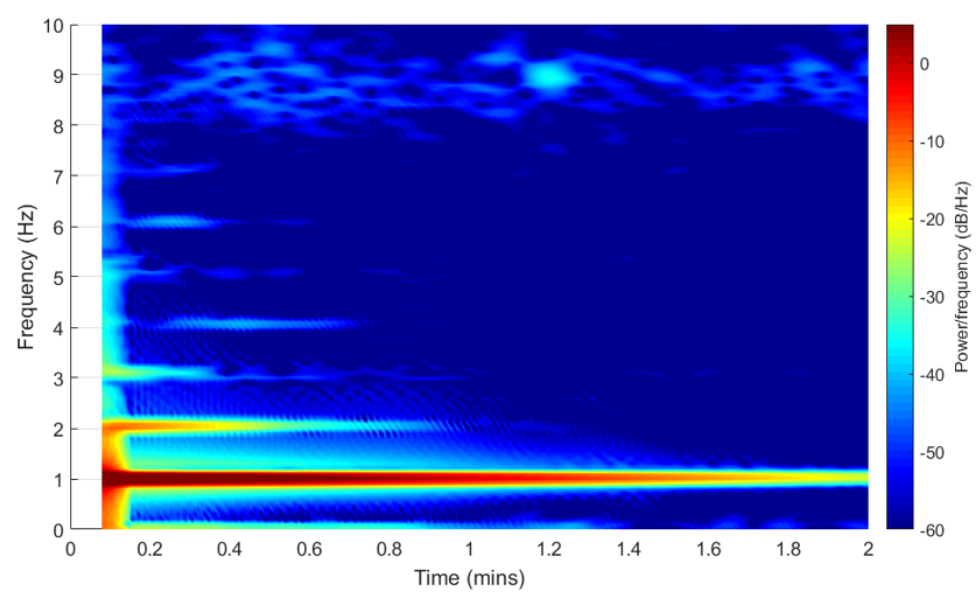

(a) First mode

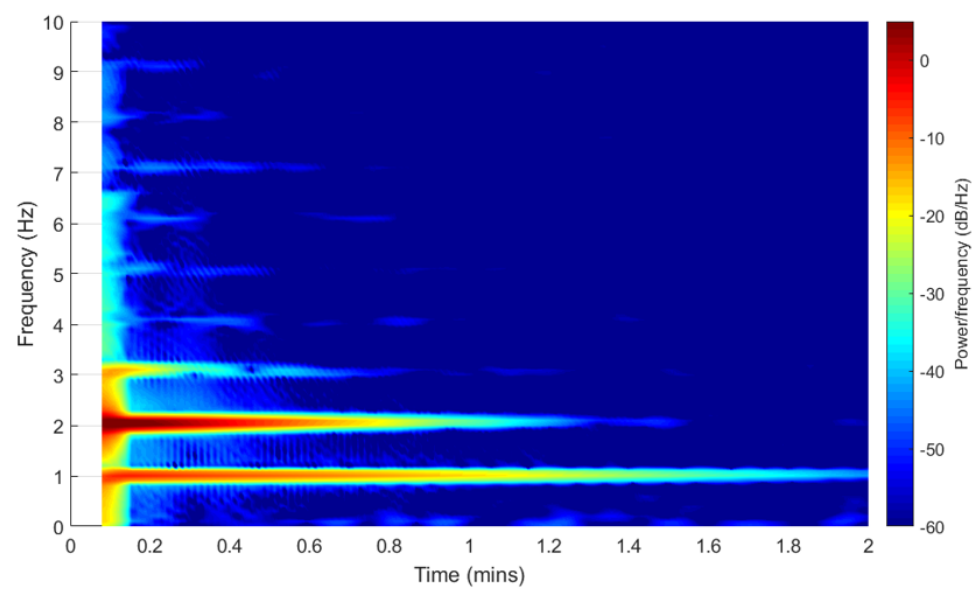

(b) Second mode

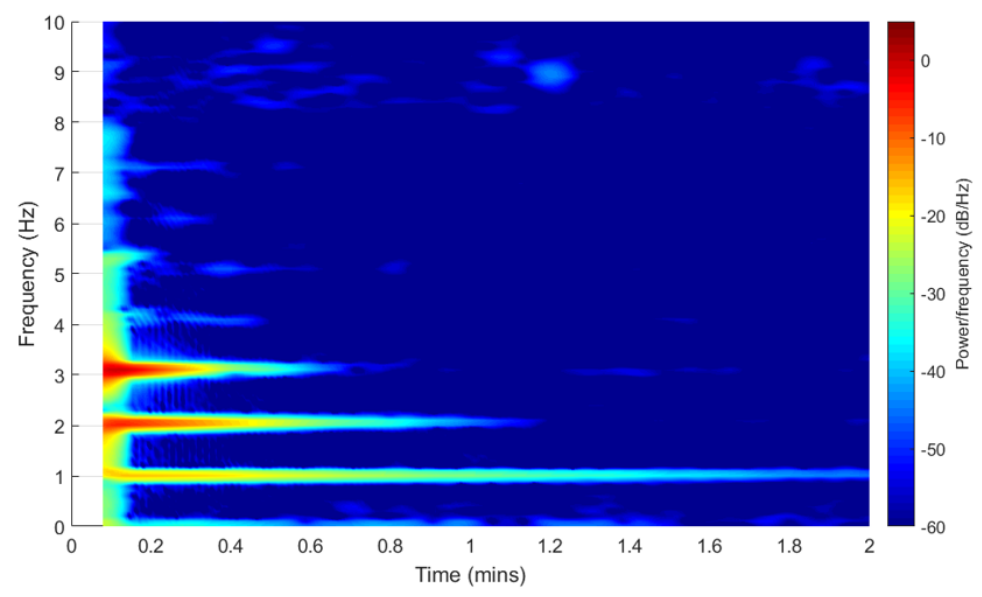

(c) Third mode 
Figure 5.24: Free-Decay Test 5 - Modal linear damping: Mode 1.
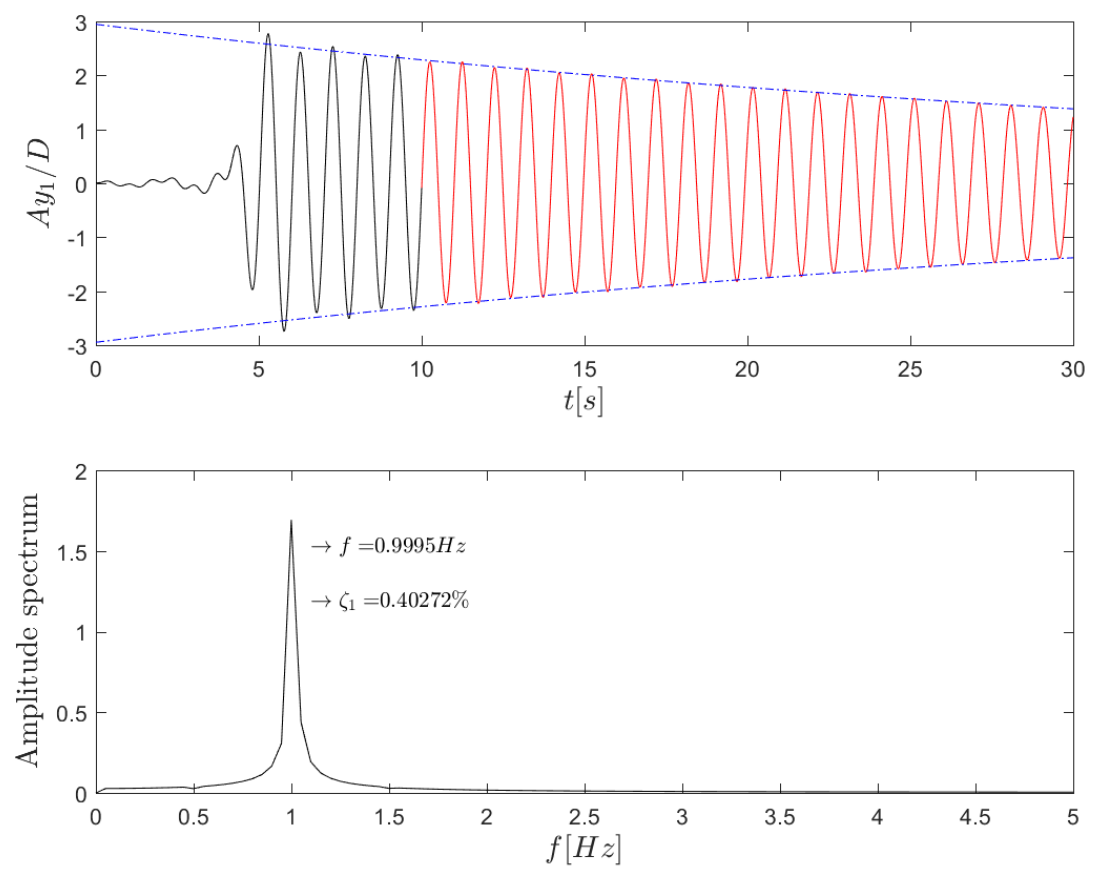

Figure 5.25: Free-Decay Test 5 - Modal linear damping: Mode 2.
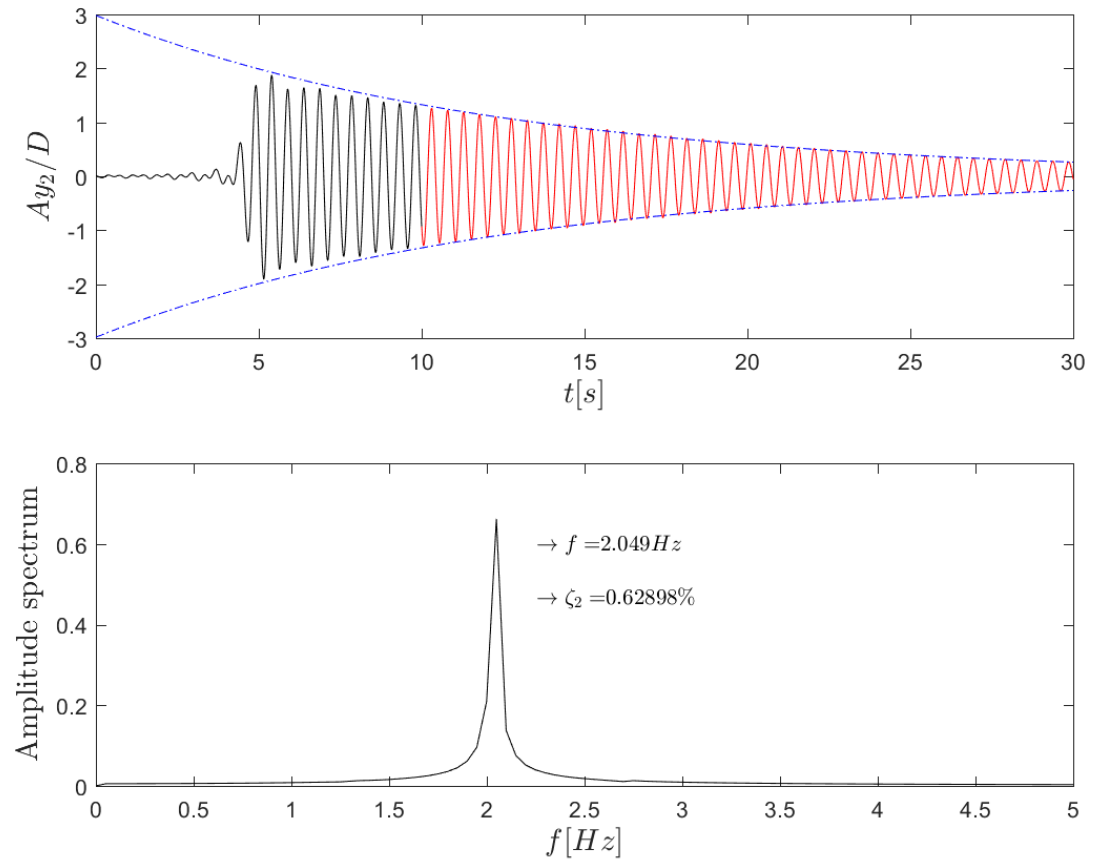
Figure 5.26: Free-Decay Test 5 - Modal linear damping: Mode 3.
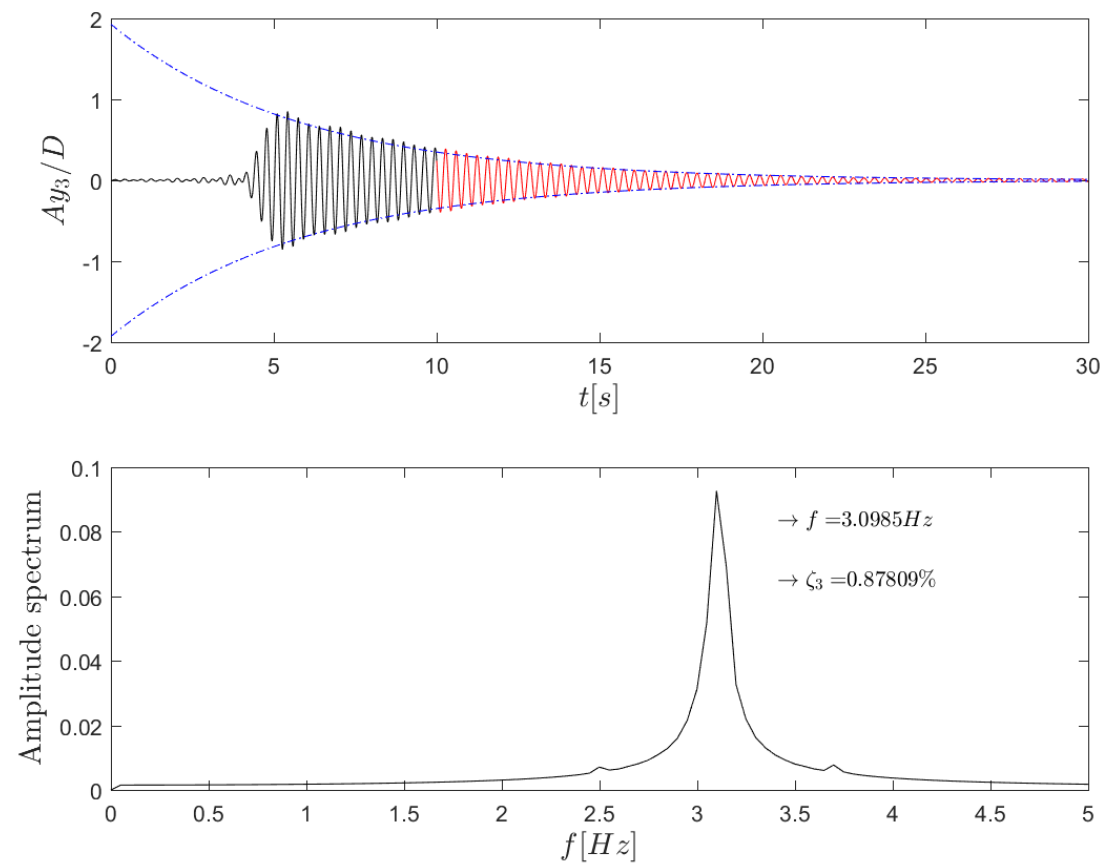
Figure 5.27: Free-Decay Test 6 - Amplitude spectrogram $\left(y^{\star}\right)$.

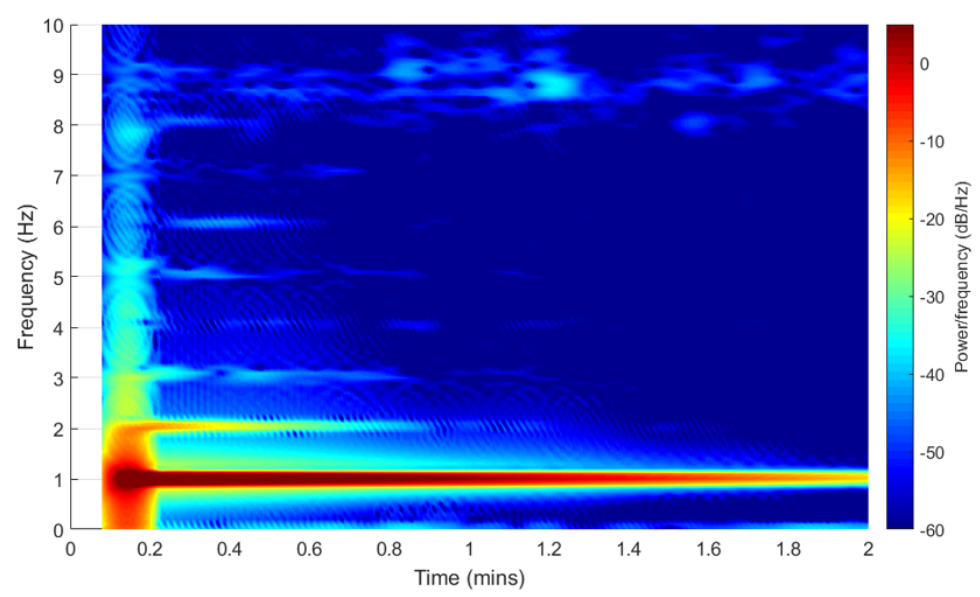

(a) First mode

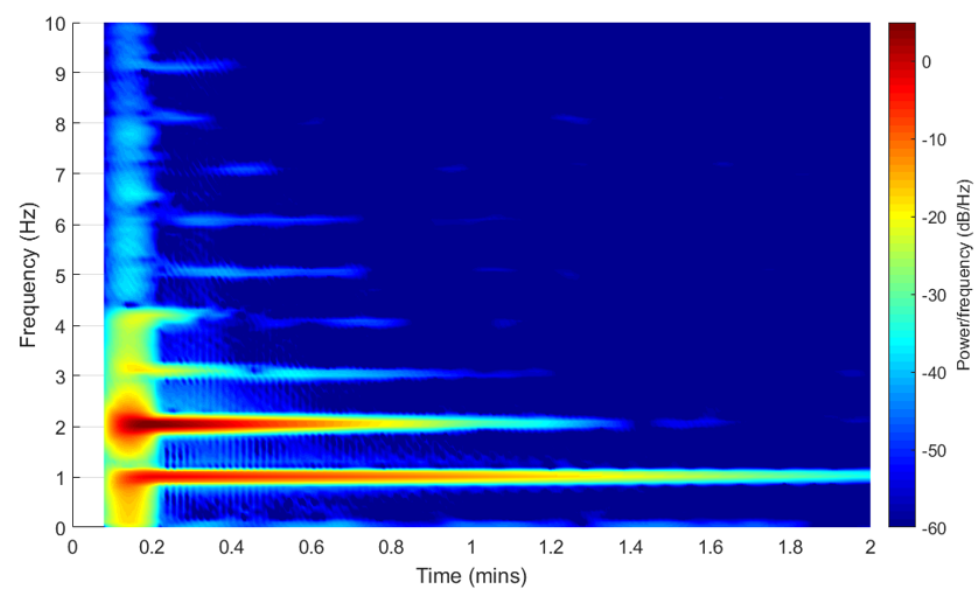

(b) Second mode

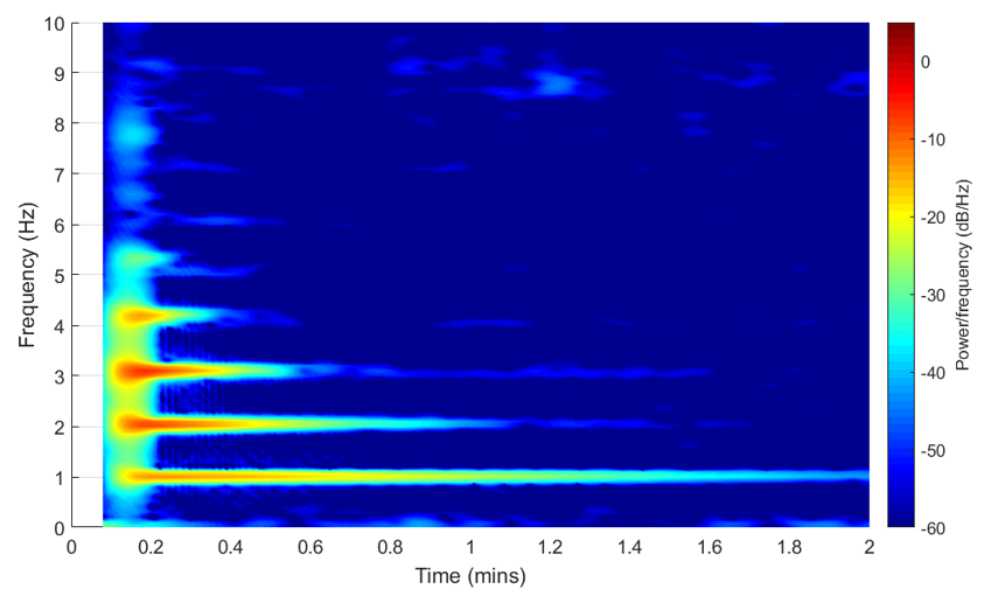

(c) Third mode 
Figure 5.28: Free-Decay Test 6 - Modal linear damping: Mode 1.
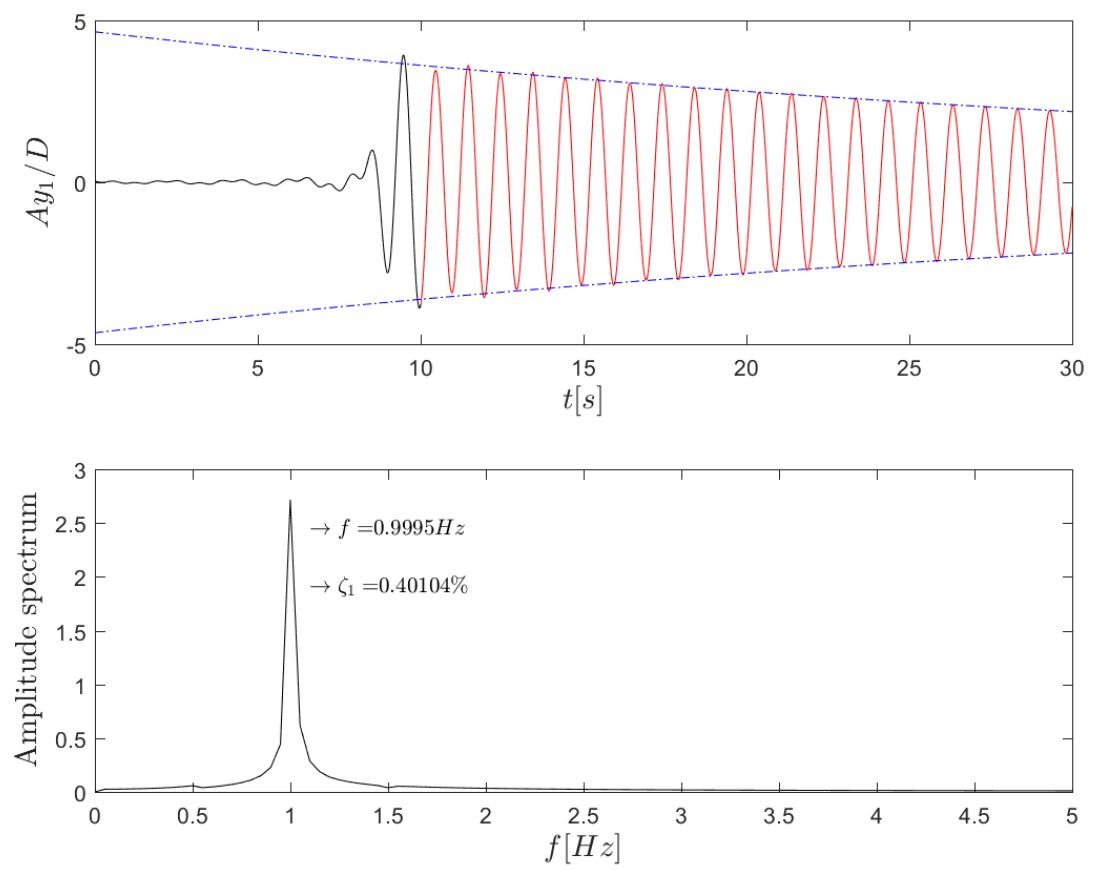

Figure 5.29: Free-Decay Test 6 - Modal linear damping: Mode 2.
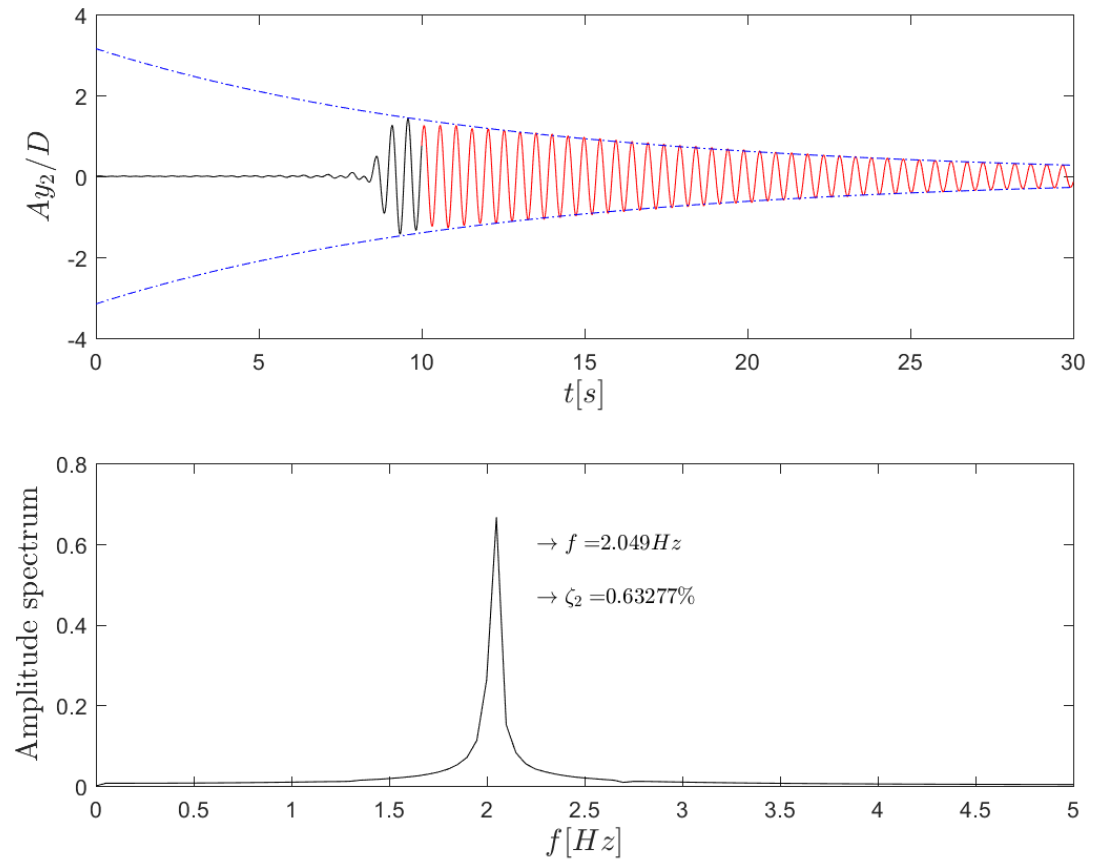
Figure 5.30: Free-Decay Test 6 - Modal linear damping: Mode 3.
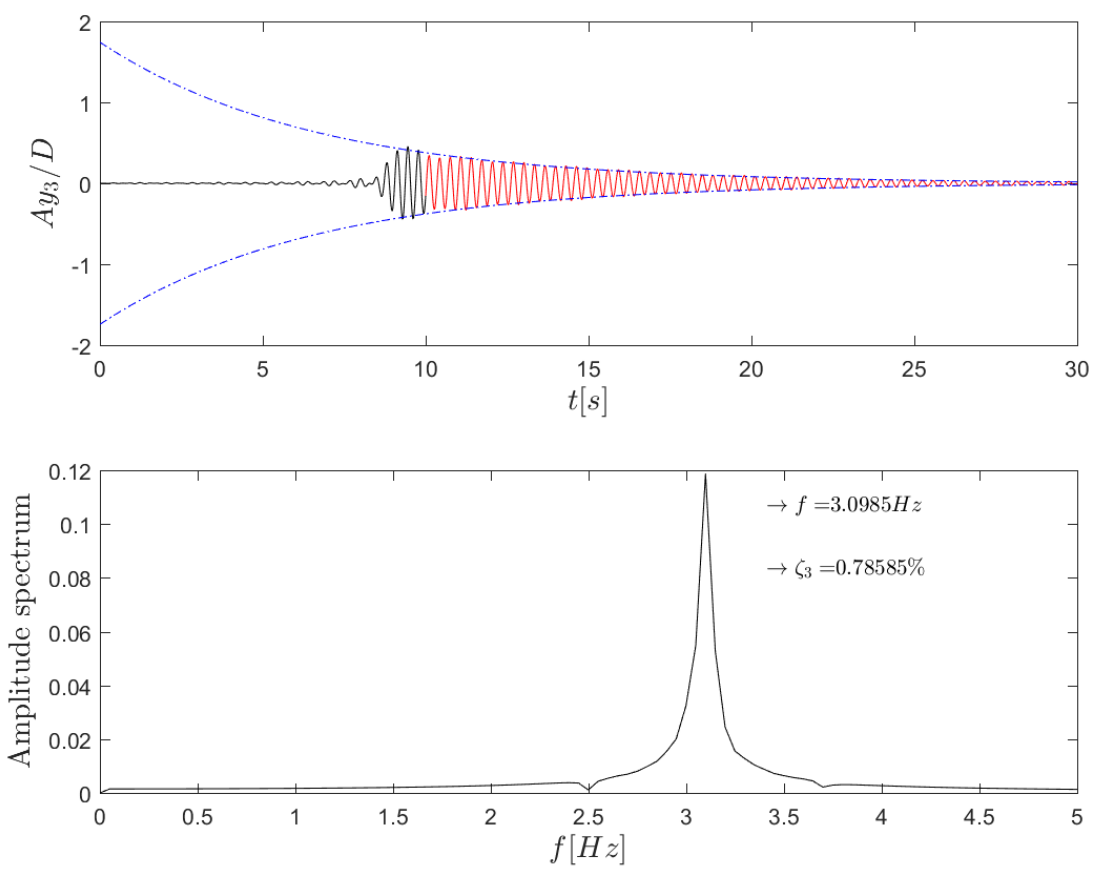

Table 5.1: Free-decay: eigenfrequencies and linear damping results - Tests: 4,5 and 6.

\begin{tabular}{cccc}
\hline & $n$ & $f_{N, n}[\mathrm{~Hz}]$ & $\zeta_{n}[\%]$ \\
\hline \multirow{4}{*}{ Test 4 } & $\underline{1}$ & 0.9995 & 0.4601 \\
& 2 & 2.0490 & 0.5607 \\
& $\underline{3}$ & 3.0985 & 0.9001 \\
& & & \\
Test 5 & $\underline{2}$ & 2.0490 & 0.6210 \\
& $\underline{3}$ & 3.0985 & 0.8781 \\
& & & \\
Test 6 & $\underline{2}$ & 2.0490 & 0.6328 \\
& 3 & 3.0988 & 0.7856 \\
\hline
\end{tabular}

Table 5.2: Mean values of frequency and linear damping considering the most suitable results from Tests 4,5 and 6.

\begin{tabular}{ccc}
\hline$n$ & $f_{N, n}[H z]$ & $\zeta_{n}[\%]$ \\
\hline 1 & 0.9995 & 0.4213 \\
2 & 2.0490 & 0.6269 \\
3 & 3.0985 & 0.8891 \\
\hline
\end{tabular}




\subsubsection{Modal Added Mass}

Resetting the added mass parameter has a direct influence on the eigenvalues of the system. Moreover, the buoyancy effect changes the immersed weight of the model, also changing some modal parameters, e.g., modal rigidity; see eq. 3.30.

It will be considered that the fundamental link between the model immersed in water and the other in air is their geometric rigidity. Using eq. 3.30 notation:

$$
\eta_{n_{w}}=\eta_{n_{a i r}}
$$

Considering only the sine eigenfunction, setting the weight function of the inner product to be unitary, $w(z)=1$; see eq. 3.24, Equation 5.1 can be stated as:

$$
\begin{aligned}
& \eta_{n_{w}}=\int_{0}^{L}\left\{\gamma_{i} \psi_{n, z}+\left[T_{t_{w}}-\gamma_{i}\right.\right.\left.(L-z)] \psi_{n, z z}\right\} \psi_{n} d z= \\
&=\int_{0}^{L}\left\{\gamma \psi_{n, z}+\left[T_{t_{a i r}}-\gamma(L-z)\right] \psi_{n, z z}\right\} \psi_{n} d z=\eta_{n_{a i r}}
\end{aligned}
$$

Using now the eigenfunction definition, $\psi_{n}(z)=\sin (n \pi z / L)$, and evaluating its first and second derivative, it is possible to perform the integral in eq. 5.2 analytically, reaching the following result shown in Table 5.3.

Table 5.3: Modal parameters eq. 3.30 analytical expressions, using sine eigenfunction base.

\begin{tabular}{ccc}
\hline Parameter & Symbol & Analytical expression \\
\hline Modal mass & $M_{n}$ & $\frac{\left(\mu+m_{a}\right) L}{2}$ \\
Modal rigidity & $\eta_{n}$ & $\left(\frac{n \pi}{2}\right)^{2}\left(\frac{2 T_{t}}{L}-\gamma\right)$ \\
Modal rigidity (modulation) & $\xi_{n}$ & $\left(\frac{n \pi}{2}\right)^{2}\left(\frac{E A}{L_{0}}\right) \frac{2 A_{t}}{L}$ \\
\hline
\end{tabular}

By definition, the immersed weight (per unit of length) is the structural weight decreased of the buoyancy effect, thus: $\gamma_{i}=\left(\mu-\pi \rho_{w} D^{2} / 4\right) g$ and $\gamma=\mu g$. Finally, together with eq. 5.3, it is possible to find the value of the traction at the top of the model which sets the same axial rigidity for both environments; see eq. 5.4.

$$
\frac{2 T_{t_{a i r}}}{L}-\gamma=\frac{2 T_{t_{w}}}{L}-\gamma_{i} \Rightarrow T_{t_{a i r}}=T_{t_{w}}+\frac{L}{2}\left(\gamma-\gamma_{i}\right)
$$




$$
T_{t_{a i r}}=T_{t_{w}}+\frac{\pi \rho_{w} g D^{2} L}{8}
$$

Regarding the model parameters, see Table 4.4, it is possible to approximately evaluate ${ }^{2}$ eq. 5.4, obtaining:

$$
T_{t_{a i r}}=45.10 \mathrm{~N}
$$

In the free decay experimental test analyzed, the tension imposed at the top time series may be seen in Fig.5.31. The mean value of the tension is $T=47.36 \mathrm{~N}$, being very close to the predicted value; see eq.5.5.

Figure 5.31: Free-Decay Test - Tension imposed at the top.

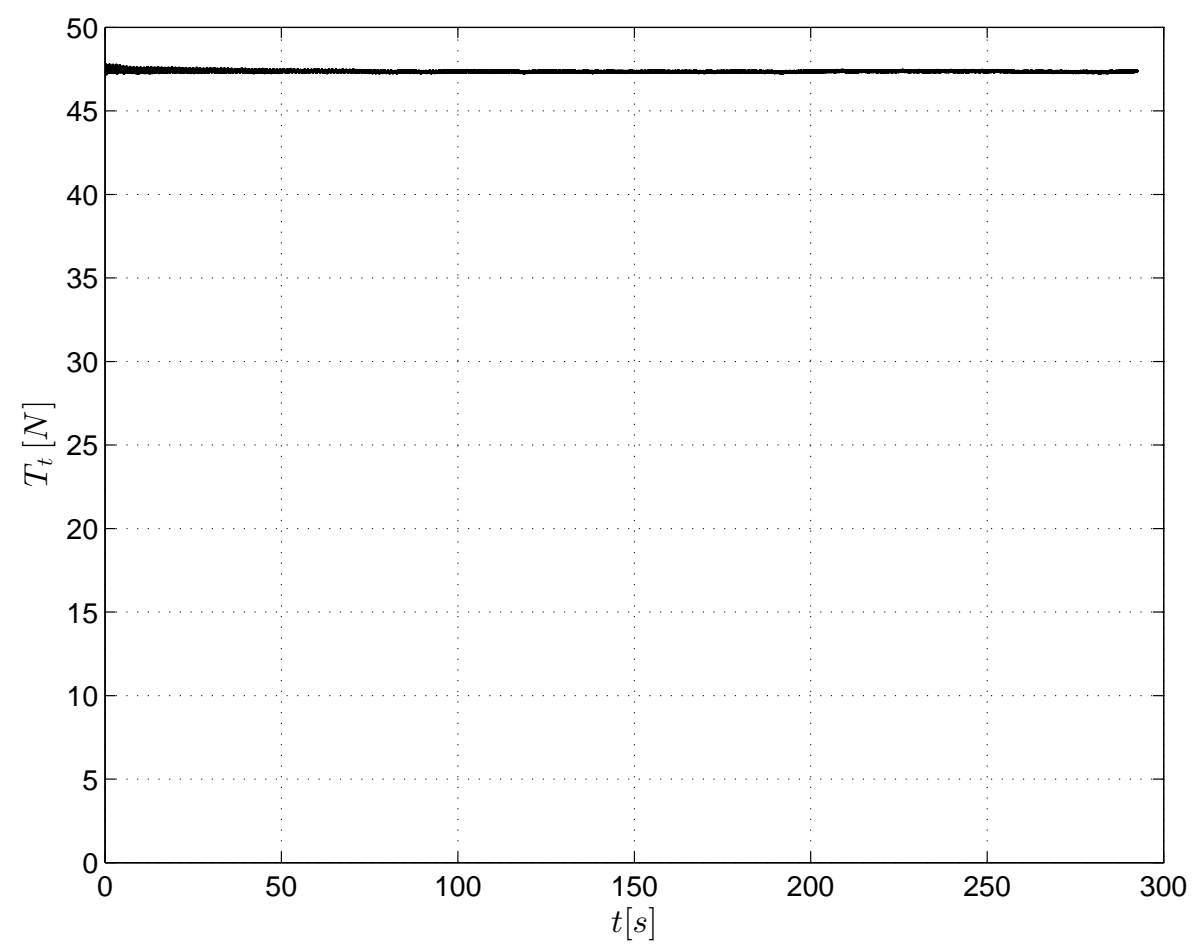

Table 5.4 shows numerical values of axial stiffness for both experimental set-ups in water and in air. The axial rigidity equivalence is shown evaluating the parameter in both cases and noting that they are equivalent mode by mode.

\footnotetext{
${ }^{2}$ In fact, given the elastic behavior of the material, the solution is iterative, since the model will stretch when solicited.
} 
Table 5.4: Axial rigidity equivalence

\begin{tabular}{ccc}
\hline Parameter & Water & Air \\
\hline$L[m]$ & 2.602 & 2.754 \\
$\gamma_{i}[N / m]$ & 7.31 & 11.13 \\
$\bar{T}_{t}[N]$ & 40 & 47.36 \\
$\eta_{1}[N / m]$ & $\underline{57.83}$ & $\underline{57.41}$ \\
$\eta_{2}[N / m]$ & $\underline{231.32}$ & $\underline{229.62}$ \\
$\eta_{3}[N / m]$ & $\underline{520.47}$ & $\underline{516.65}$ \\
\hline
\end{tabular}

Furthermore, using the axial rigidity equivalence, the modal added mass is a parameter easy to obtain from eq. 5.1, and Table 5.3.

$$
\begin{gathered}
\eta_{n_{\text {air }}}=\eta_{n_{w}} \Leftrightarrow\left(\frac{\omega_{N, n_{w}}}{\omega_{N, n_{a i r}}}\right)^{2}=\frac{M_{n_{\text {air }}}}{M_{n_{w}}} \text { with } M_{n_{w}}=M_{n_{w}}^{\text {struc }}+M_{n_{w}}^{a} \text { and } M_{n_{\text {air }}}=M_{n_{w}}^{\text {struc }} \\
\therefore M_{n_{w}}^{a}=M_{n}^{a}=C_{a_{n}} M_{n}^{d}=M_{n_{w}}^{\text {struc }}\left[\left(\frac{\omega_{N, n_{a i r}}}{\omega_{N, n_{w}}}\right)^{2}-1\right]
\end{gathered}
$$

Regarding the chosen eigenfunction and the definition of $M_{n}$, see eqs. $3.30 \& 5.6$, it follows:

$$
\begin{gathered}
M_{n}=\frac{\left(\mu+m_{a}\right) L}{2} \\
M_{n}^{d}=\frac{\pi \rho_{w} D^{2} L}{8} \\
M_{n}^{s t r u c}=\frac{\mu L}{2} \\
C_{a_{n}}=\frac{4 \mu}{\pi \rho_{w} D^{2}}\left[\left(\frac{\omega_{N, n_{a i r}}}{\omega_{N, n_{w}}}\right)^{2}-1\right]=\frac{4 \mu}{\pi \rho_{w} D^{2}}\left[\left(\frac{f_{N, n_{a i r}}}{f_{N, n_{w}}}\right)^{2}-1\right]
\end{gathered}
$$

The added mass coefficient defined in eq. 5.7 is a relation between the added mass and the fluid displaced mass from the body, $m_{a}=C_{a} m_{d}$. Alternatively, the added mass coefficient may be defined as a relation between added mass and the structure mass: 


$$
\begin{gathered}
M_{n}^{a}=a_{n} M_{n}^{\text {struc }}=a_{n} M_{n}^{\text {struc }} \frac{M_{n}^{d}}{M_{n}^{d}} \\
C_{a_{n}}=\frac{M_{n}^{a}}{M_{n}^{d}}=a_{n} \frac{M_{n}^{\text {struc }}}{M_{n}^{d}}=a_{n} M_{n}^{\star} \\
a_{n}=\frac{C_{a_{n}}}{M_{n}^{\star}}
\end{gathered}
$$

Another way to estimate the modal added mass coefficient is directly evaluate it from the Eigenvalue definition, considered the natural frequency known from experiments, as in eq. 5.9.

$$
\begin{gathered}
\left(\omega_{N, n}^{w}\right)^{2}=\frac{\eta_{n}}{M_{n}}=\frac{\eta_{n}}{\left(1+\tilde{a}_{n}\right) M_{n}^{\text {struc }}} \\
\tilde{a}_{n}=\frac{2 \eta_{n}}{\mu L\left(\omega_{N, n}^{w}\right)^{2}}-1=\frac{2 \eta_{n}}{\mu L\left(2 \pi f_{N, n}^{w}\right)^{2}}-1 \\
\tilde{a}_{n}=\frac{2 \eta_{n}}{\mu L\left(2 \pi f_{N, n}^{w}\right)^{2}}-1
\end{gathered}
$$

Amplitude spectrum density charts, see Figs.5.12-5.14, show which dominant frequencies coexist in the analyzed signal. The data is organized in Tab.5.5, as follows.

Table 5.5: Modal added mass

\begin{tabular}{cccccc}
\hline$n$ & $f_{N, n}^{w}[H z]$ & $f_{N, n}^{a}[H z]$ & $C_{a_{n}}$ & $a_{n}$ & $\tilde{a}_{n}$ \\
\hline 1 & 0.84 & 0.9995 & 1.2246 & 0.4158 & 0.4201 \\
2 & 1.68 & 2.0490 & 1.4859 & 0.4875 & 0.4014 \\
3 & 2.52 & 3.0985 & 1.5074 & 0.5118 & 0.3998 \\
\hline
\end{tabular}

Analyzing Table 5.5 added mass coefficients $a_{n}$ and $\tilde{a}_{n}$ evaluated from different methods, two behaviors come to attention. The first is quite immediate that the added mass $a_{n}$ increases with higher modes. On the other hand, the added mass $\tilde{a}_{n}$ decreases.

Both approaches lead to an equivalent result for the first mode, but the discrepancy of others results only increases higher the next mode is. The coefficient order is the same in both methods.

Considering the experiments presented in Sarpkaya (1977) with a rigid cylinder in a constant oscillatory flow, non-dimension numbers as Reynolds $(R e)$, 
Keulegan-Carpenter $(\mathrm{KC})$ and the frequency parameter $\left(\beta_{K C}\right)^{3}$ give a hint of what should be obtained for added mass coefficient and drag coefficient.

4

The free-decay test is non-stationary, so it is outside the experiments described in Sarpkaya (1977). On the other hand, the free-decay is asymptotic to the trivial equilibrium response and there is a possibility to find a initial range of the inertial coefficient in order to inspect consistency with respect to the results shown in Table 5.5.

Figure 5.32: Hydrodynamics coefficients vs. Keulegan-Carpenter number for constant values of the frequency parameter and Reynolds number

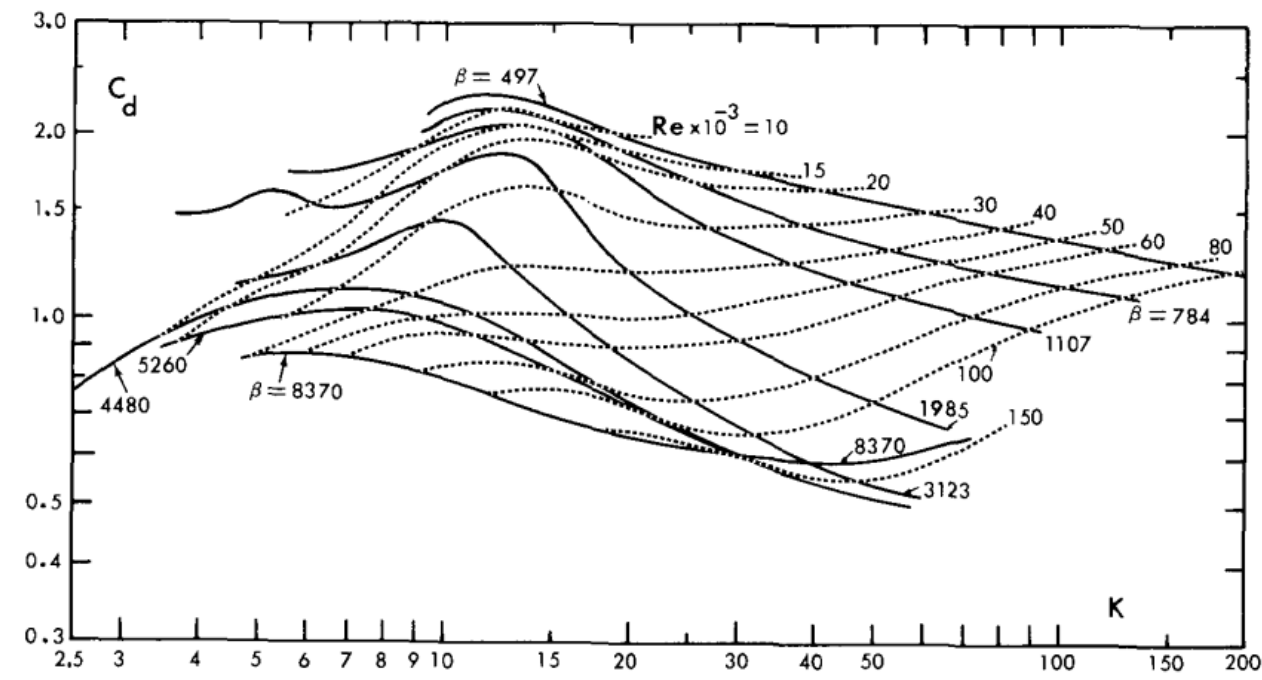

(a) Drag coefficient, $C_{d}$

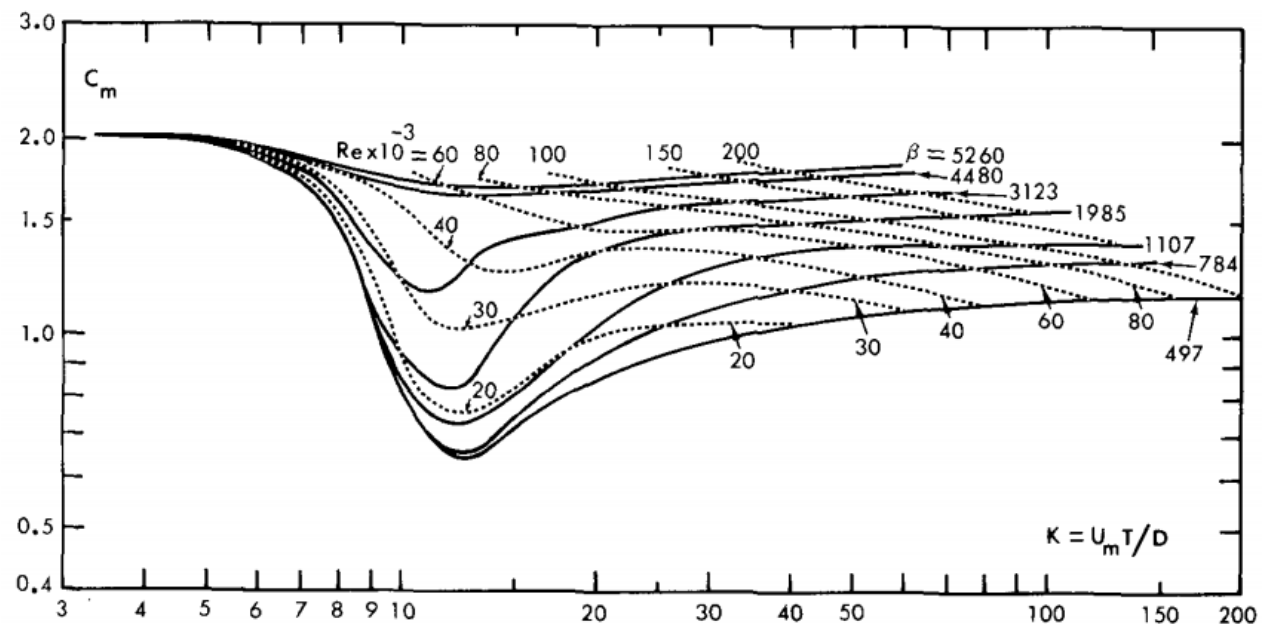

(b) Inertia coefficient, $C_{m}=C_{a}+1$

Source: Extracted from Sarpkaya (1977).

\footnotetext{
${ }^{4}$ In Figures 5.32a-b, $\beta_{K C}$ is called $\beta$. However, the present defines $\beta_{K C}$ the frequency parameter in order to avoid any confusion due to $\beta$ is been used for others purposes in the present text.
} 
If a monochromatic response is considered, $y(t)=A \sin \left(2 \pi f_{N} t\right)$, it leads to:

$$
\begin{aligned}
R e & =\frac{U_{\max } D}{\nu}=\frac{A D \omega_{N}}{\nu} \\
K C & =\frac{U_{\max }}{f_{N} D}=\frac{2 \pi A}{D} \\
\beta & =\frac{R e}{K C}=\frac{D^{2} f_{N}}{\nu}
\end{aligned}
$$

Figures 5.33a-b shown a typical free-decay in water. Choosing a modal amplitude of approximately $A y_{1} / D=0.05$ and $A y_{1} / D=0.04$, the KC number order is less than the unity $\left(\mathcal{O}(K C)=10^{-1}\right)$. Looking at Figure 5.32, the $C_{m}$ curve is asymptotic near 2 to low $\mathrm{KC}$ number and all Re range considered. The inertia coefficient indicates that $C_{a_{N}} \approx 1$ for both modes $\left(C_{m}=C_{a}+1\right)$. 
Figure 5.33: Normalized amplitude of free-decay test in water

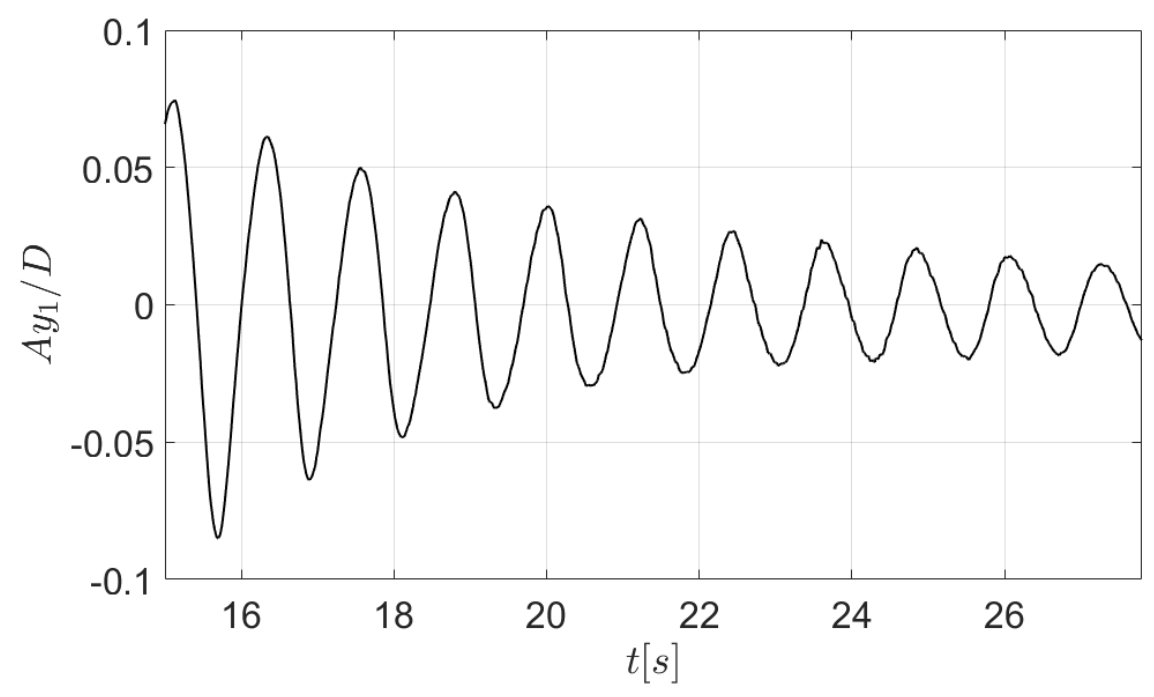

(a) First mode, $A y_{1} / D$

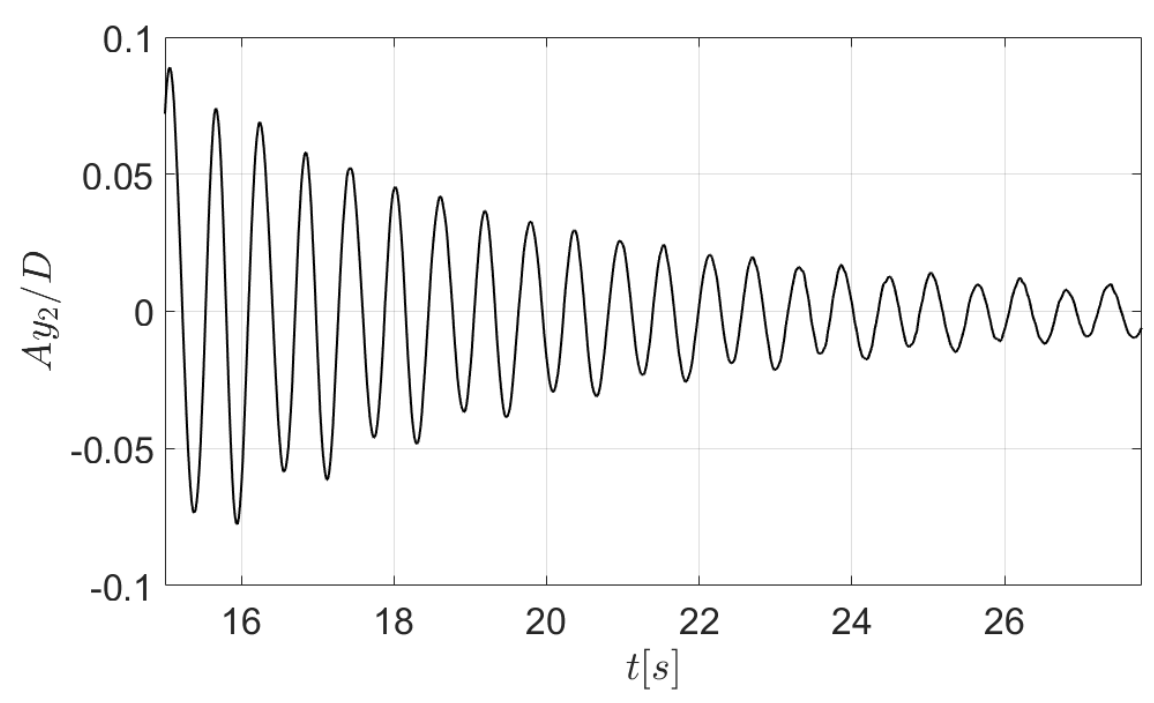

(b) Second mode, $A y_{2} / D$

Such result appears, at a first analysis, non consistent with the added mass coefficient presented in Table 5.5. In fact, the added mass evaluated from both experimental approaches are greater than the unity suggested by Sarpkaya (1977). Nevertheless, Pesce and Fujarra (2000) have also found an added mass value for a flexible cantilever greater than the unity $\left(C_{a, 1}=1.17\right)$. Thorsen et al. (2016), analyzing experimental data of a flexible cylinder subjected to an oscillatory flow presented by Fu et al. (2014), also found added mass coefficient value greater than the unity $\left(C_{a}=1.53\right)$. It should be recalled that the Sarpkaya charts were obtained for rigid cylinders under oscillatory flow, not for flexible cylinders. 


\subsection{Parametric resonance and Mathieu instability}

This section is dedicated to explore the possibility to occur a modal Mathieu instability due to imposed heave motion. Mathieu instability is caused by a bifurcation on the dynamical system, in which the stable center turns into unstable focus with a limit cycle (Hopf bifurcation) for the trivial solution.

The Strutt diagram relates the Mathieu oscillator parameters, see eqs. 3.32-3.33, into a chart-guide which can predict whether the system will be on a stable or unstable region for the trivial solution. The Strutt diagram is determined by a linear oscillator, with or without an equivalent damping term. However what would be the effects of considering a non-linear oscillator with a quadratically damping term, as the hydrodynamic drag?

This question might be answered by parts, using local pertubation techniques. Ramani et al. (2004) presented an approach in which, by considering the quadratically damping term, the Strutt diagram shows a second bifurcation, see Figure 5.34, determined by the red line. This line is evaluated using a local perturbation method and the bifurcation line is better defined when closer to the point $P$. The point $P$ is determined in the critical point where the derivative change its signal in the Strutt diagram first stability tongue. 
Figure 5.34: Second bifurcation on Strutt diagram by introducing a quadratically damping term.

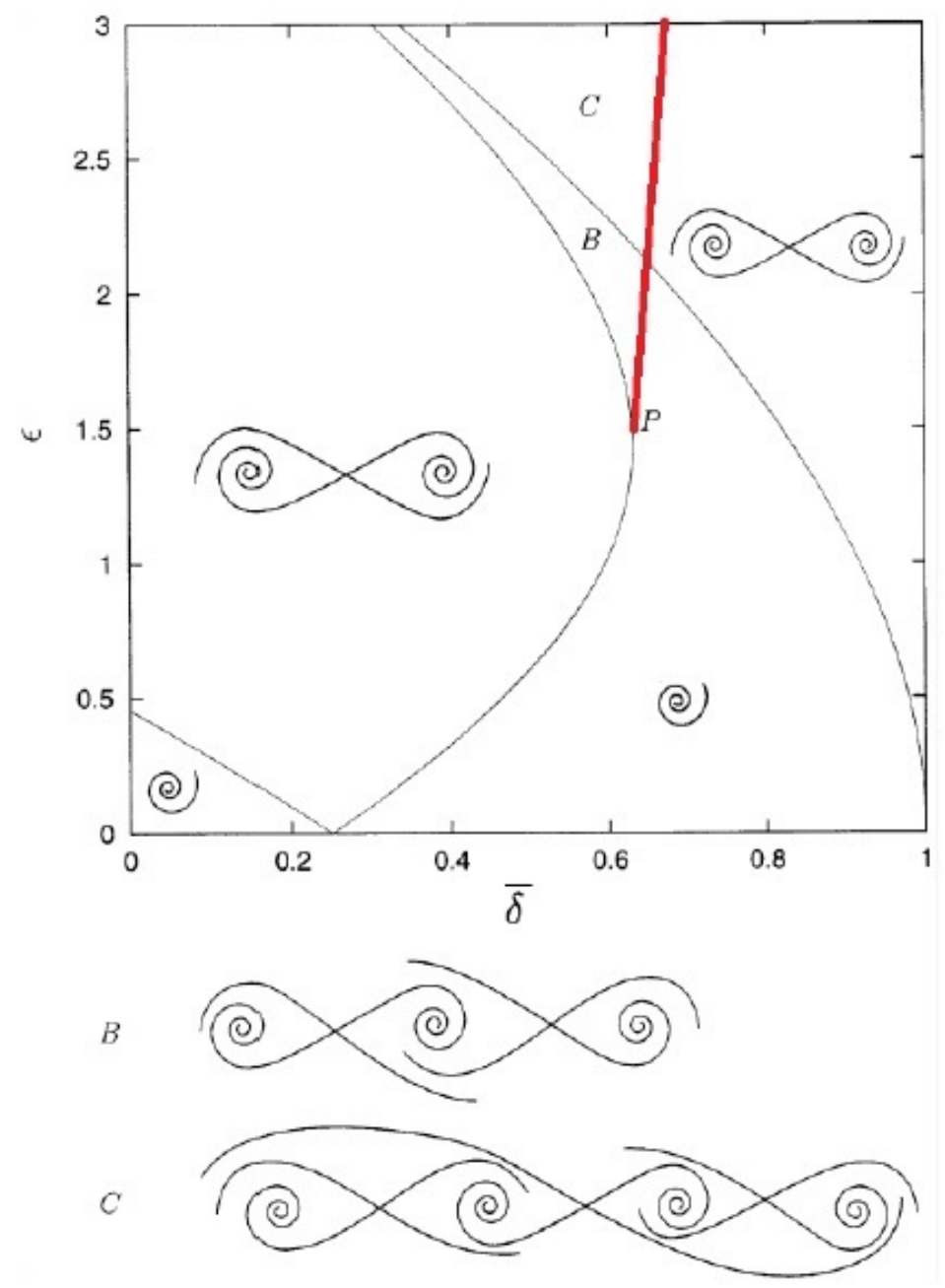

Source: Adapted from Ramani et al. (2004), Franzini et al. (2014) \& Franzini et al. (2015).

The modal oscillator presented in Ramani et al. (2004) uses $\bar{\delta}=\left(\frac{\omega_{N}}{\omega_{t}}\right)^{2}$. The difference between $\bar{\delta}$ and $\delta$ defined in eq. 3.32 is a factor of four, leading to a Strutt chart equivalent, however in a different scale.

The "correction" provided by the method is interesting, inasmuch as, for a linear system, whether it is located in the area above the second bifurcation line, but outside the tongues, the Strutt diagram would predict a stable behavior for the trivial solution; instead, the quadratically damping term causes the same region to be unstable.

Considering the Mathieu-Hill oscillator in eq. 3.33, the Strutt diagram seen in fig. 5.35 shows that the bifurcation occurs every time the system leaves the dashed area. Considering the tongue tips at the line $\varepsilon=0$, it is possible to determine the relation between driven frequency and natural frequency necessary to leads the system into a Mathieu instability analyzing the oscillator $\delta$-parameter. 
Figure 5.35: Strutt diagram of classical undamped Mathieu-Hill oscillator.

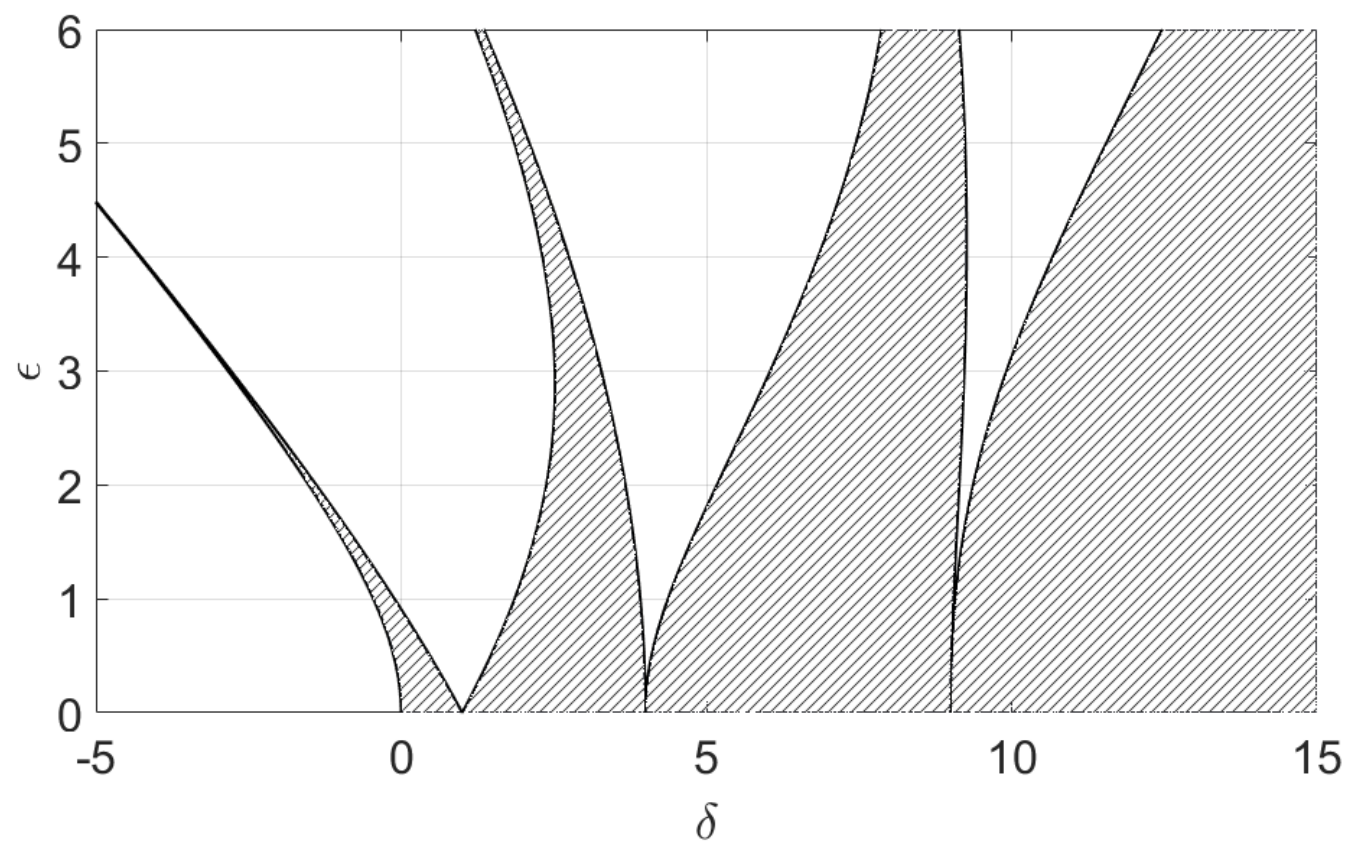

The Mathieu instability occurs every time the following relation is given:

$$
\begin{gathered}
\delta_{n}=\left(\frac{2 \omega_{N, 1}}{\omega_{t}}\right)^{2}=k^{2} \text { with } k \in \mathbb{N} \\
\left(\frac{2 \omega_{N, 1}}{\omega_{t}}\right)^{2}=1 \Longrightarrow 2 \omega_{N, 1}=\omega_{t} \\
\left(\frac{2 \omega_{N, 1}}{\omega_{t}}\right)^{2}=4 \Longrightarrow \omega_{N, 1}=\omega_{t} \\
\left(\frac{2 \omega_{N, 1}}{\omega_{t}}\right)^{2}=9 \Longrightarrow 2 \omega_{N, 1}=3 \omega_{t}
\end{gathered}
$$

Among the imposed heave experiments analyzed, there are cases in which the relation between the driven and natural frequencies are $f_{t}: f_{N, 1}=1: 1, f_{t}: f_{N, 1}=2: 1$ and $f_{t}: f_{N, 1}=3: 1$. In $f_{t}: f_{N, 1}=1: 1$ and $f_{t}: f_{N, 1}=2: 1$ cases, whether the trivial solution becomes unstable, it will be caused by a Mathieu instability; otherwise any other instability can not be Mathieu-type. The modal Strutt diagrams for the flexible vertical cylinder in water can be seen in fig. 5.36. 
Figure 5.36: Modal Strutt diagrams approximated to a Mathieu oscillator with quadratically damping function. Heave: Red square $-f_{t}: f_{N, 1}=1: 1$; Green square $-f_{t}: f_{N, 1}=2: 1$; \& Blue square $f_{t}: f_{N, 1}=3: 1$.

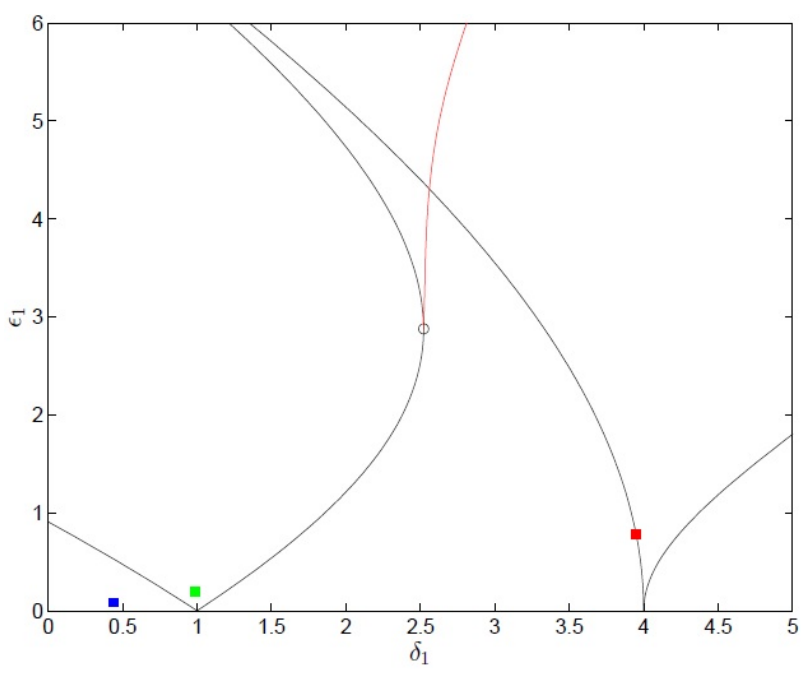

(a) First mode.

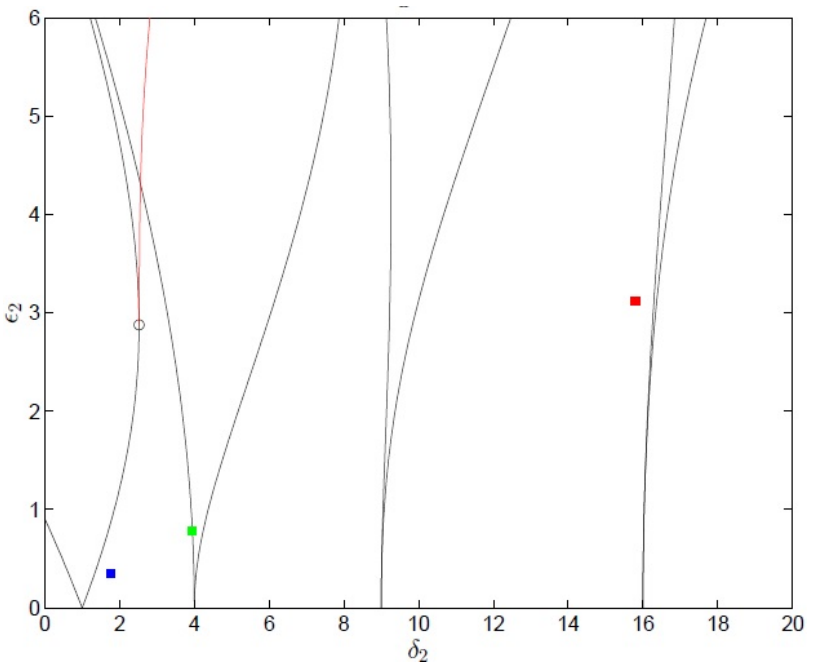

(b) Second mode.

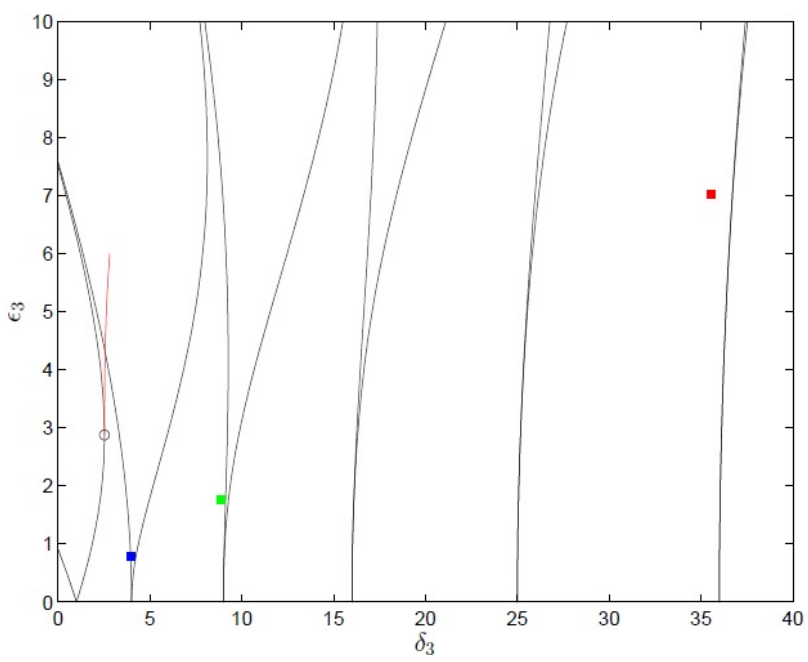

(c) Third mode.

Source: Extracted from Franzini et al. (2014) \& Franzini et al. (2015). 
Considering the first mode, the reader can identify the blue square data $\left(f_{t}: f_{N, 1}=3\right.$ : $1)$ in Fig. 5.36a. Only the red square $\left(f_{t}: f_{N, 1}=1: 1\right)$ and green square $\left(f_{t}: f_{N, 1}=2: 1\right)$ are to be considered as Mathieu instability if they are inside a unstable region. A close look can assure that both points are inside a unstable region. Therefore the trivial solutions for first mode in the $f_{t}: f_{N, 1}=1: 1$ and $f_{t}: f_{N, 1}=2: 1$ parametric excitation are unstable.

The second mode eigenfrequency $\omega_{N, 2} \approx 2 \omega_{N, 1}$, inasmuch as the model in water holds the Pythagorean harmonic series. In that case only there is only one Mathieu instability due to the $f_{t}: f_{N, 1}=2: 1$ that corresponds to the $f_{t}: f_{N, 2}=2: 2$; the green square in Figure 5.36b.

The same procedure for the third mode leads to $\omega_{N, 3} \approx 3 \omega_{N, 1}$ and, among the driven frequencies, only $f_{t}: f_{N, 1}=3: 1\left(f_{t}: f_{N, 3}=3: 3\right)$ and $f_{t}: f_{n, 1}=2: 1\left(f_{t}: f_{N, 3}=2: 3\right)$ are Mathieu instabilities of the trivial solution; respectively, the blue square and green square in Figure 5.36c.

The results obtained with the Strutt diagram is summed up in Table 5.6. Those results obtained are consistent with the power spectra shown in Figures 5.2, 5.3, 5.4c-d where the trivial solution is unstable and the system finds another periodic solution for each mode. The composition of modes provides scalograms as shown in Figures $5.4 \mathrm{a}-\mathrm{b}$ in which the coexistence of more than one mode in the elastica dynamics can be verified.

Table 5.6: Modal Strutt diagram stability of the trivial solution for experiments in water

\begin{tabular}{cccc}
\hline $\mathrm{n}$ & \multicolumn{3}{c}{$f_{t} / f_{N, n}$} \\
\cline { 2 - 3 } & $1: 1$ & $2: 1$ & $3: 1$ \\
\hline 1 & $\underline{\text { Unstable }}$ & $\underline{\text { Unstable }}$ & Stable \\
2 & Stable & Unstable & Stable \\
3 & Stable & Unstable & Unstable \\
\hline
\end{tabular}

Introducing Reduce Order Models (ROMs) similar to the ROM described in eq. 3.32, Franzini et al. (2015) has reached to numerical simulations of each mode, as seen in Figures 5.37-5.39; see Table 5.7.

Table 5.7: Parameters of Mathieu-Hill oscillators (ROMs) studied in Franzini et al. (2015)

\begin{tabular}{cccccccc}
\hline \multirow{2}{*}{$f_{t} / f_{N, 1}$} & \multicolumn{4}{c}{$\delta_{n}$} & & \multicolumn{3}{c}{$\varepsilon_{n}$} \\
\cline { 2 - 4 } \cline { 6 - 8 } & 1 & 2 & 3 & & 1 & 2 & 3 \\
\hline $1: 1$ & 3.95 & 15.80 & 35.54 & & 0.78 & 3.12 & 7.02 \\
$2: 1$ & 0.99 & 3.95 & 8.89 & & 0.20 & 0.78 & 1.76 \\
$3: 1$ & 0.44 & 1.76 & 3.95 & & 0.09 & 0.35 & 0.78 \\
\hline
\end{tabular}

Comparing the results obtained by means of Strutt diagrams and the response of the ROMs gives some interesting findings about the instability of the trivial solution when the Strutt diagram is not clear enough, e.g., regions near the bifurcation. Furthermore, 
multifrequency response composed of subharmonics are also found, a result that the Strutt diagrams could not anticipate. Quoting Franzini et al. (2015):

Another interesting finding is that, whenever the Strutt diagram did not clearly predict a single periodic solution for a given mode, the spectral analysis revealed a multifrequency response in that mode. In other words, periodic solutions emerging from Mathieu-like instabilities appear to dominate other possible harmonic components in a given mode. (FRANZINI ET AL., 2015, p. 11).

In the first mode, see Figure 5.37, there are periodic solutions for $f_{t} / f_{N, 1}=1: 1$ and $f_{t} / f_{N, 1}=2: 1$, as predicted in Table 5.6. Also as expected by Strutt diagram analysis, the $f_{t} / f_{N, 1}=3: 1$ is stable.

The second mode is a little more interesting as there is a subharmonic response, see Figure 5.38. There are periodic solutions for $f_{t} / f_{N, 1}=2: 1\left(f_{t} / f_{N, 2}=2: 2\right)$ and $f_{t} / f_{N, 1}=3: 1\left(f_{t} / f_{N, 2}=3: 2\right)$ as also predicted by the Strutt diagram. In addition, there is a quasi-periodic solution for $f_{t} / f_{N, 1}=1: 1\left(f_{t} / f_{N, 2}=1: 2\right)$ in which the power spectrum shows a composition of the harmonic $f_{r} / f_{N, 2}=2: 2$ with a subharmonic $f_{r} / f_{N, 2}=1 / 2$.

Figure 5.39 shows results for the third mode. By Strutt diagram analysis, the $f_{t} / f_{N, 1}=$ $2: 1\left(f_{t} / f_{N, 3}=2: 3\right)$ and $f_{t} / f_{N, 1}=3: 1\left(f_{t} / f_{N, 1}=3: 3\right)$ trivial solutions are known to be unstable. Besides, subharmonics $f_{r} / f_{N, 3}=1 / 3$ and $f_{r} / f_{N, 3}=2 / 3$ can be seen in the $f_{t} / f_{N, 1}=1: 1$ case; and a subharmonic $f_{r} / f_{N, 3}=4 / 3$ is associated with the $f_{t} / f_{N, 3}=2: 3$ in the response of $f_{t} / f_{N, 1}=2: 1$.

The results obtained using Strutt diagram analysis and the spectral analysis of ROMs response are summarized in Table 5.8 . 
Table 5.8: Frequency ratios of periodic response. Prediction by Strutt diagram versus determined from spectral analysis. The symbol "-" indicates stability of the trivial solution $u_{r}=0 ; f_{r}$ refers to the spectral components present in the response; * indicates negligible amplitudes — see, e.g., Figures 5.37-5.39

\begin{tabular}{|c|c|c|c|c|c|c|c|c|}
\hline \multirow[b]{2}{*}{$n$} & \multirow[b]{2}{*}{$f_{t} / f_{N, 1}$} & \multirow[b]{2}{*}{$f_{t} / f_{N, n}$} & \multicolumn{2}{|c|}{$f_{r} / f_{N, 1}$} & \multicolumn{2}{|c|}{$f_{r} / f_{t}$} & \multicolumn{2}{|c|}{$f_{r} / f_{N, n}$} \\
\hline & & & Strutt & Spectral & Strutt & Spectral & Strutt & Spectral \\
\hline \multirow{4}{*}{1} & $1: 1$ & $1: 1$ & $1: 1$ & $1: 1$ & $1: 1$ & $1: 1$ & $1: 1$ & $1: 1$ \\
\hline & $2: 1$ & $2: 1$ & $1: 1$ & $1: 1$ & $1: 2$ & $1: 2$ & $1: 1$ & $1: 1$ \\
\hline & $3: 1$ & $3: 1$ & - & $3: 1^{*}$ & - & $1: 1^{*}$ & - & $3: 1^{*}$ \\
\hline & $1: 1$ & $1: 2$ & - & $1: 1,2: 1$ & - & $1: 1,2: 1$ & - & $1: 2,1: 1$ \\
\hline \multirow[t]{2}{*}{2} & $2: 1$ & $2: 2$ & $2: 1$ & $2: 1$ & $1: 1$ & $1: 1$ & $1: 1$ & $1: 1$ \\
\hline & $3: 1$ & $3: 2$ & - & $3: 1^{*}$ & - & $3: 3^{*}$ & - & $3: 2^{*}$ \\
\hline \multirow{3}{*}{3} & $1: 1$ & $1: 3$ & - & $1: 1,2: 1$ & - & $1: 1,2,1$ & - & $1: 3,2: 3$ \\
\hline & $2: 1$ & $2: 3$ & - & $2: 1,4: 1$ & - & $1: 1,2: 1$ & - & $2: 3,4: 3$ \\
\hline & $3: 1$ & $3: 3$ & $3: 1$ & $3: 1$ & $1: 1$ & $1: 1$ & $1: 1$ & $1: 2$ \\
\hline
\end{tabular}


Figure 5.37: Amplitude series, frequency spectra (FFT) and phase portraits for the first mode.
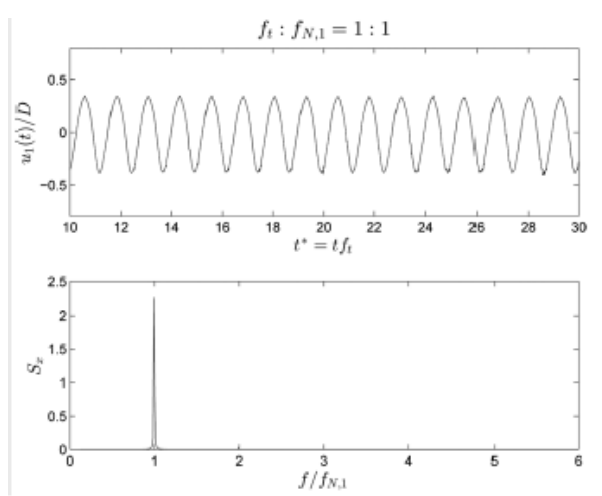

(a) $f_{t}: f_{N, 1}=1: 1$
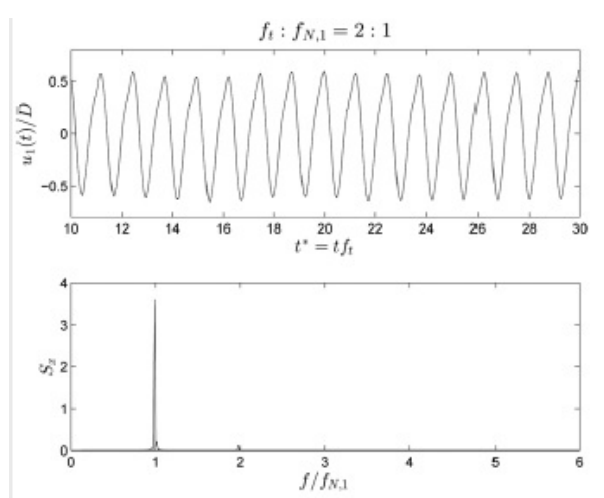

(b) $f_{t}: f_{N, 1}=2: 1$
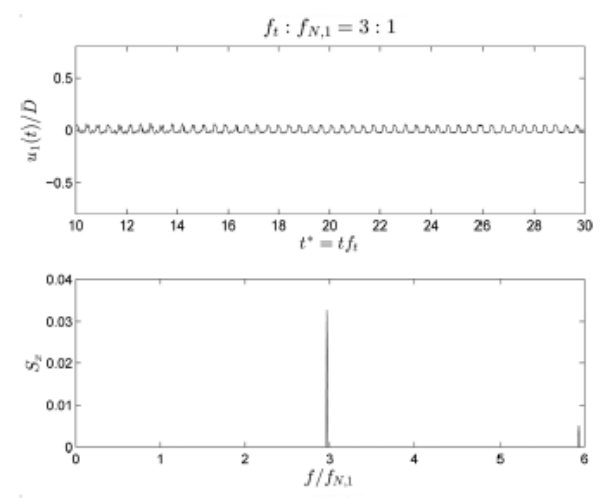
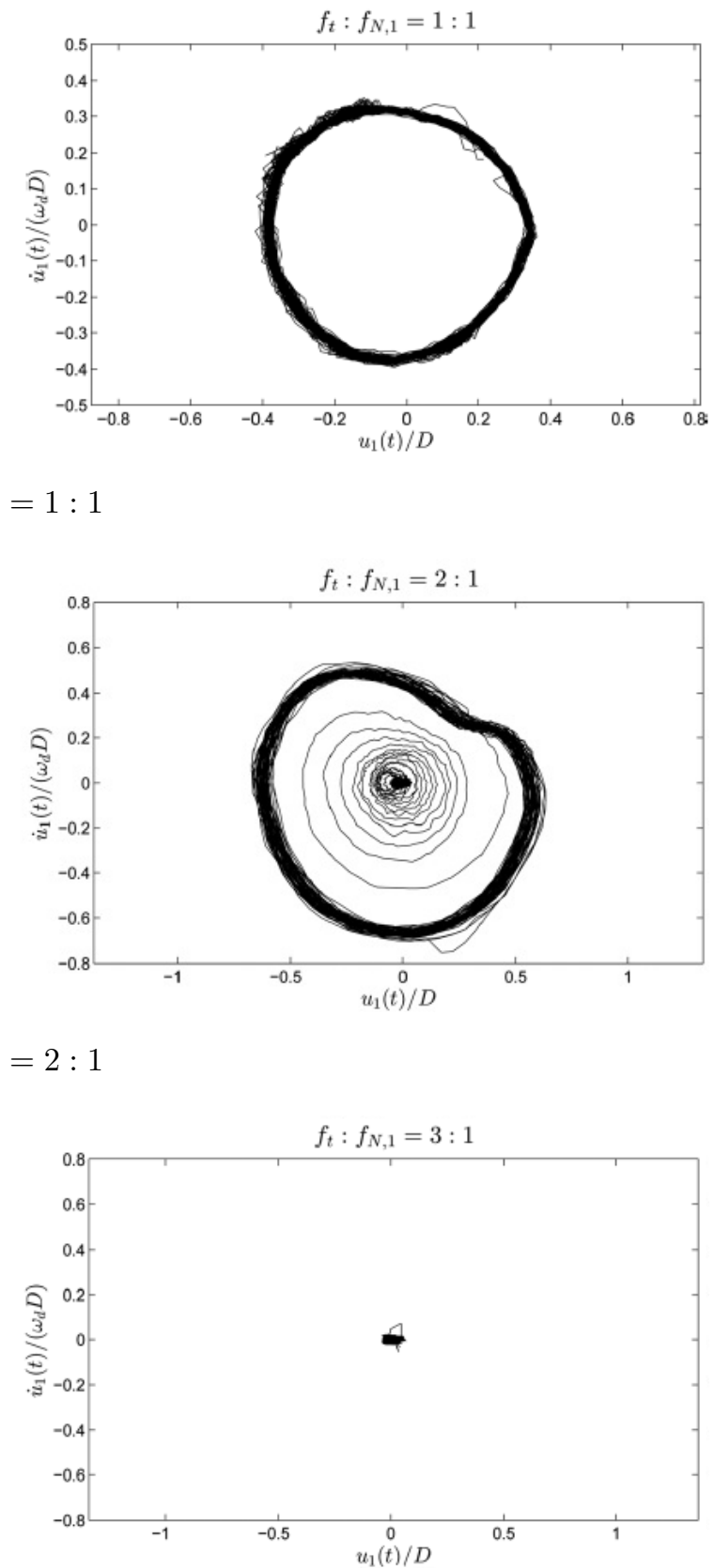

(c) $f_{t}: f_{N, 1}=3: 1$

Source: Extracted from Franzini et al. (2015). 
Figure 5.38: Amplitude series, frequency spectra (FFT) and phase portraits for the second mode.
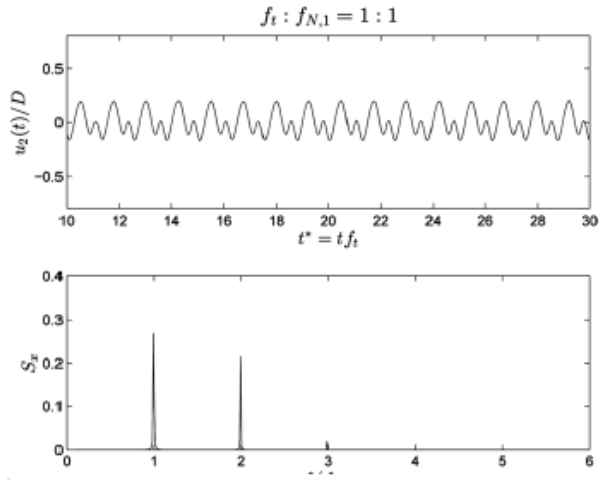

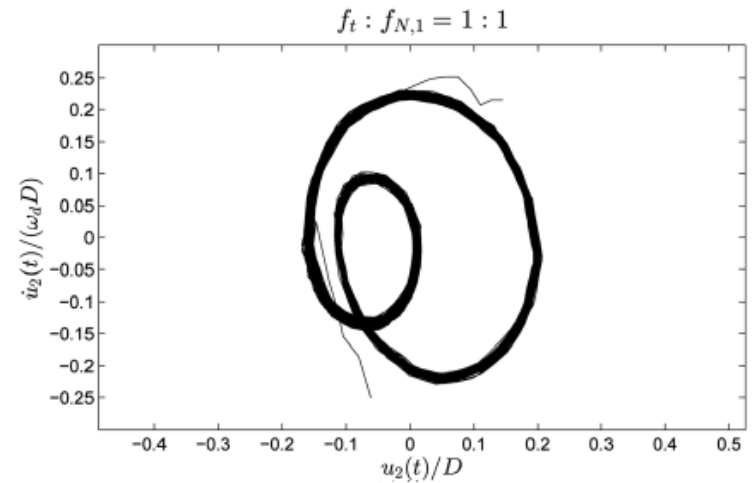

(a) $f_{t}: f_{N, 1}=1: 1$

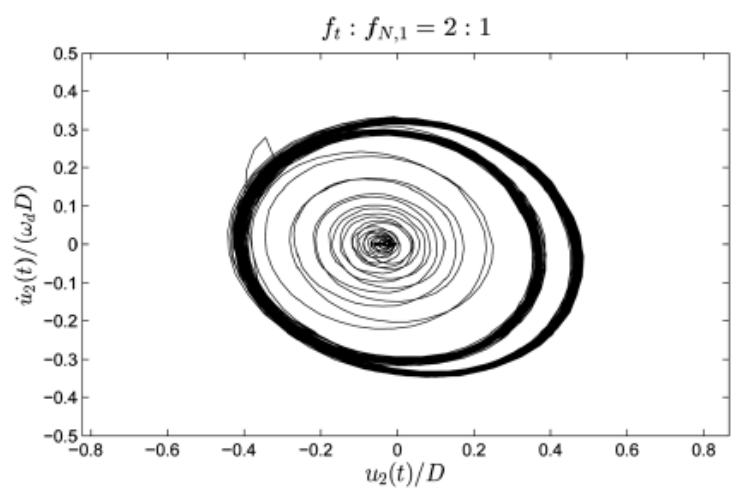

(b) $f_{t}: f_{N, 1}=2: 1$

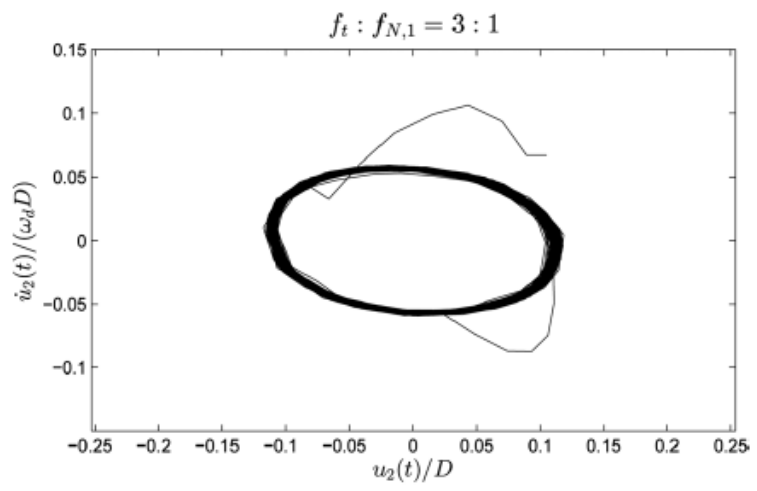

(c) $f_{t}: f_{N, 1}=3: 1$

Source: Extracted from Franzini et al. (2015). 
Figure 5.39: Amplitude series, frequency spectra (FFT) and phase portraits for the third mode.
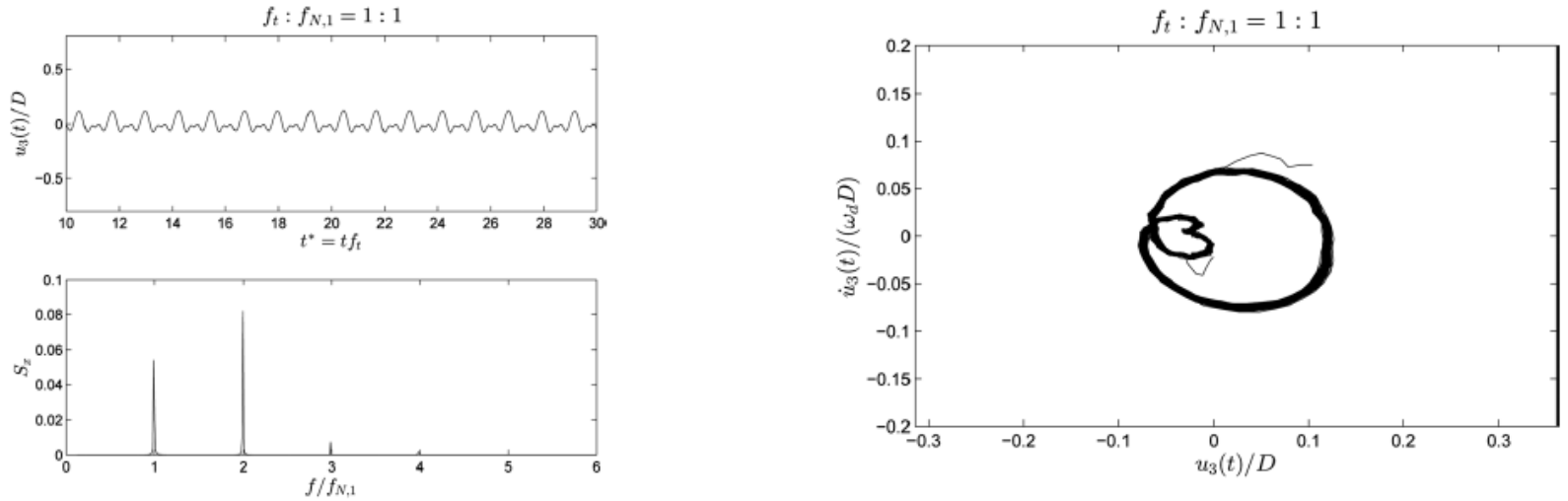

(a) $f_{t}: f_{N, 1}=1: 1$
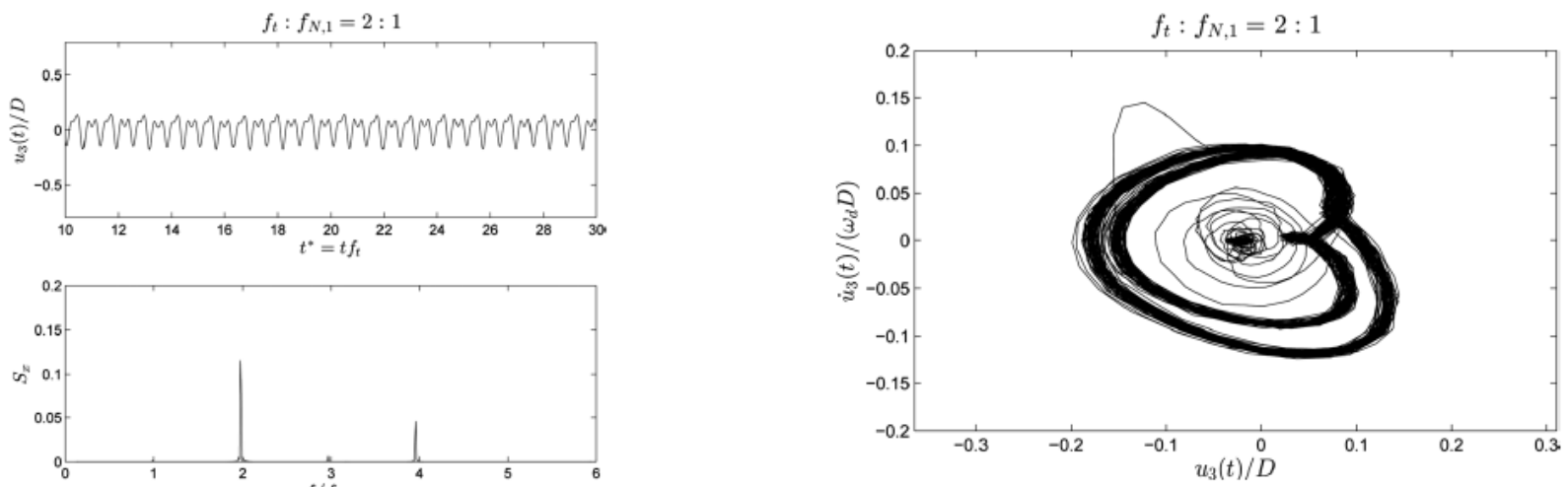

(b) $f_{t}: f_{N, 1}=2: 1$
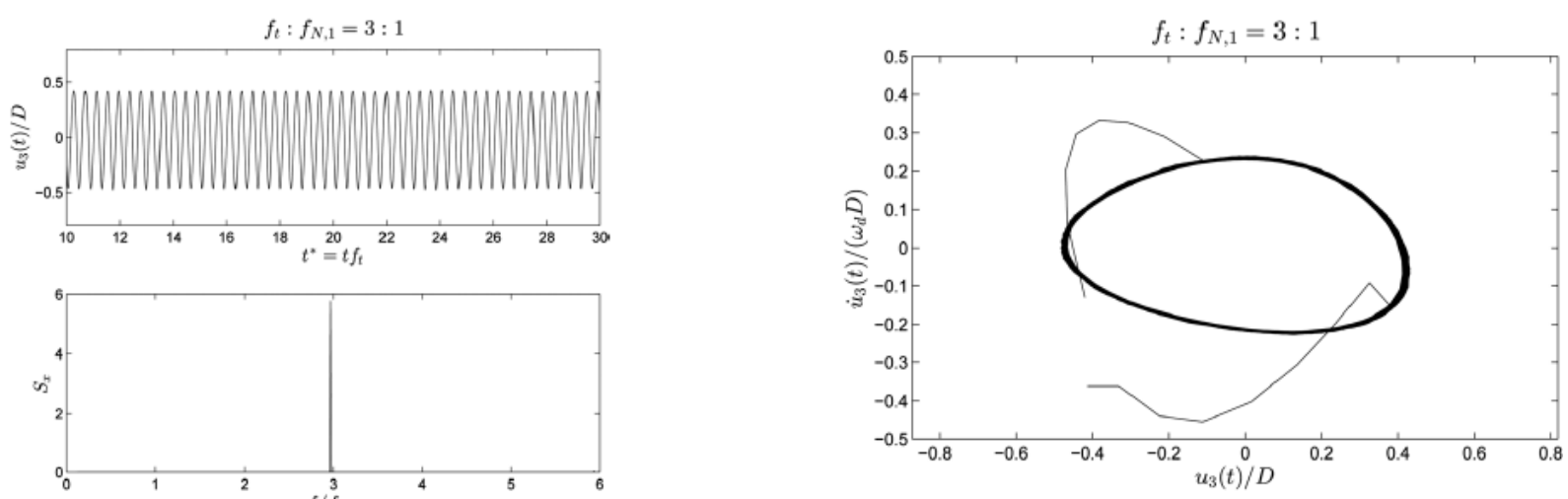

(c) $f_{t}: f_{N, 1}=3: 1$

Source: Extracted from Franzini et al. (2015). 


\section{Chapter 6}

\section{Conclusions}

Alongside the experimental tests with a vertical flexible cylinder immersed in water described in LIFE\&MO (2012d), a new experimental test using the same model in similar conditions was performed in order to obtain parameters, as: structural modal damping coefficients and modal added mass coefficient.

For the sake of simplicity, the modal decomposition chosen used eigenfunctions based on a trigonometric base. The results obtained by using the trigonometric base were both satisfactory in the experiments in water and in air.

The experimental test conducted in air provided, successfully, modal structural damping, when subjected to a free-decay test. The modal damping coefficients are shown in Tab.5.2. These coefficients will be of importance in further analysis in order to understand how much damping is caused by the structure itself, or by the drag force in water. Understanding the difference between structural and hydrodynamical damping is crucial, inasmuch as the structural damping is considered to be proportional do the relative velocity of the structure, while the hydrodynamic damping is essentially non-linear.

The most interesting result obtained using the free-decay test in air was the evaluation of the modal added mass coefficient in still water. Such prediction is said to be in still water, since it would be necessary a different experimental procedure to capture damping during a towing-tank run, by studying transitory responses.

As discussed before, there are some important points to be considered in the added mass results. The first is the difference between the increasing $\left(a_{N}\right)$ or decreasing $\left(\tilde{a}_{N}\right)$ behavior of added mass coefficient with increase of the mode number. Such behavior could not be explained with the present work. Additional analysis, including numerical models, could be used in order to verify which of the results are more consistent. However the first mode added mass is equivalent in both approaches used, $a_{1}=0.4158 \approx 0.4201=$ $\tilde{a}_{1}$.

The second important consideration to be done about the added mass coefficient refers to Sarpkaya (1977) results for a rigid cylinder under the effect of a oscillatory flow 
around it. Sarpkaya showed that the inertia coefficient has a asymptotic value $\left(C_{m}=\right.$ $1+C_{a} \approx 2, C_{a} \approx 1$ ) for low KC number at any Reynolds number considered.

The added mass, $C_{a}$ found in Table 5.5 is greater than the unity predicted by Sarpkaya. However another experiment with a flexible structure presented in Pesce and Fujarra (2000) has also shown a added mass coefficient greater than the unity. The case presented in Pesce and Fujarra (2000) is a cantilever and the added mass was evaluated using a relation between natural frequencies in air and in water likewise performed in the present work.

The discussion about the value of flexible cylinder added mass coefficient is not a closed subject. In fact, the recent work by Thorsen et al. (2016), e.g., on flexible cylinders in oscillating flows, still uses $C_{a}=1$ in its proposed empirical hydrodynamic model. The experimental work by Fu et. al (2014), where a flexible cylinder is transversally excited with sinusoidal displacements applied at the extremities, does not investigate the modal added mass, also using $C_{a}=1$.

Franzini et al. (2016), using nonlinear oscillators obtained through the Galerkin's projection technique, using sinusoidal modes, investigate the response sensitivity of the vertical flexible cylinder subjected to parametric excitation to the parameters $C_{D}$ and $C_{a}$, varied within a significant interval.

In the present work the modal added mass coefficients, $C_{a}$, are larger than unity, the asymptotic limit when KC goes to zero for rigid cylinders subjected to oscillatory flows; Sarpkaya (1977).

Reasons for that are not clear at this point and could be related to the choice of the modal basis. A more represantive basis, as that called quasi-Bessel, could lead to dinstinct results; see Dias (2015).

It should be emphasized that the methods applied in the present work to obtain the modal added mass coefficients rely on decay tests only. Such point is essential in the definition of representative KC and Reynolds numbers, inasmuch as the amplitude varies in the free-decay and many of the hydrodynamic coefficients are function of the amplitude.

Parametric resonance and Mathieu instability of the heave imposed experimental tests in water were also explored in the present analysis. Strutt diagram predicted unstable trivial solutions for some modes, leading to periodic solutions. The Mathieu instability was discussed in the previous chapter; see p. 71.

Numerical results obtained with reduced-order models in Franzini et al. (2015) show that, even in situations when the Strutt diagram does not predict instability of the trivial solution, a response dominated by subharmonics is possible, leading the system to a new quasi-periodical modal amplitude solution; see Table 5.8.

The presence of dominant subharmonic response in cases in which Strutt diagram did not predict any stable periodical solution is a very interesting result found. This is closely 
linked to the process of choosing a modal basis in order to decompose the movement. The trigonometric base used has monochromatic modes and the experimental analysis indicates the presence of subharmonics, associating the structure frequency modes with the driven frequency.

In fact, the discussion on the proper modal basis choice permeates the whole analysis. The Galerkin criteria must hold for any Eigen function chosen and the trigonometric modes are, in fact, the simplest possible. The finite number of modes necessary in order to reconstruct the elastica dynamics would decrease with a better choice of modal shapes. Quasi-Bessel modes appear as a good choice, since written in a closed-form and they already including non-linear effects of curvature and elongation; see Mazzilli et al. (2014).

As further topics of research, a few are mentioned in the following paragraphs.

Similar to the study of parametric resonance and Mathieu instability of the experiments with imposed parametric heave in water, there is a database of experiments in air with parametric imposed heave. The difference between being immersed in water and air will be to enlarge the Strutt diagram unstable tongue regions as effect of the damping be greater in water than in air. Such difference might bring more subharmonics to the response, or cause parametric resonance in modes that were stable in water.

It is also possible to use a non-linear Reduced Order Model (ROM) in order to evaluate the effect of some hydrodynamical coefficients, as added mass and non-linear damping; Franzini et al. (2015) and Franzini et al. (2016). It would be interesting to compare the results obtained using the analytical-numerical ROM with the experimental results in water obtained with modal decomposition techniques.

The use of Floquet theory and evaluation of characteristic exponents to study the stability of trivial solution of a modal Mathieu-Hill oscillator can also provide a new methodology to predict when the bifurcation will occur in some mode for a certain imposed heave, complementary to the use of Strutt diagram.

Other possible inroad to take is using some better representation base for the modal decomposition. The quasi-Bessel base has a closed-form solution, being very easy to be adapted to the modal decomposition algorithms. Some advantages of the quasi-Bessel mode was already mentioned, but it is very useful to point out again that those modes inherited properties of a non-linear beam model, which is more robust than any cable model using trigonometric ou classical Bessel modes; see Mazzilli et al. (2014).

Numerical models (CFD) could be used in order to assess the added mass experimental estimates. Besides, numerical models could lead to a full characterization of hydrodynamics coefficients in a wide range of Reynolds number and Keulegan-Carpenter number. 
Fu et al. (2014) and Thorsen et al. (2016) present interesting results of hydrodynamic forces on a flexible cylinder. Further experiments in order to assess the inertia coefficient would assist towards a deeper understanding of added mass results obtained in the present work.

Another topic that may be addressed is the experimental tests with incident flow, dealing with concomitant fluid-structure phenomena, as Vortex Induced Vibration and parametric resonance. This can be carried out by analysing the enormous experimental data base already available from the experiments at IPT; see LIFE\&MO (2012d).

In the present work, only the vertical flexible cylinder was studied. However, as presented in the Preface, p. xxi, experimental tests were also carried out for "catenary" configurations at the IPT facility; see LIFE\&MO (2012c). Pereira (2014) has already carried out an extensive experimental analsyis ov VIV of "catenary" configurations, by using Galerkin's projections on proper numerically determined modes. Further modal analysis with the "catenary" model in order to assess hydrodynamics coefficients, numerical calibration of modal phenomenological models, parametric resonance and Mathieu instabilities, as well as the effects of modal decomposition using non-linear modes. 


\section{References}

Abramowitz, M. and I.A. Stegun. 1972. Handbook of mathematical functions: With formulas, graphs, and mathematical tables, Applied mathematics series, Dover Publications.

Aranha, J.A.P., C.A. Martins, and C.P. Pesce. 1997. Analytical approximation for the dynamic bending moment at the touchdown point of a catenary riser, International Journal of Offshore and Polar Engineering 7, no. 4.

Assi, G.R.S. 2009. Mechanisms for flow-induced vibration of interfering bluff bodies, Ph.D. Thesis, Imperial College, London, England.

Bowman, F. 1958. Introduction to Bessel Functions, Dover Books on Mathematics, Dover Publications.

Casetta, L. and C.P. Pesce. 2013. The generalized Hamilton's principle for a non-material volume, Acta Mechanica 224, no. 4, 919-924. doi 10.1007/s00707-012-0807-9.

Chatjigeorgiou, I.K. 2008a. Application of the WKB method to catenary-shaped slender structures, Mathematical and Computer Modelling 48, 249-257.

$2008 \mathrm{~b}$. Solution of the boundary layer problem for calculating the natural modes of riseriser slender structures, Journal of Offshore Mechanics and Arctic Engineering 130.

Dias, T. 2015. Análise da Instabilidade Paramétrica de Risers Retos via Modelo de Ordem Reduzida Baseado em Modo Não Linear com Função do Tipo Bessel, Master's Thesis, Escola Politécnica da Universidade de São Paulo, São Paulo, Brazil. In Portuguese.

Dugas, R. 1988. A history of mechanics, Dover Books on Physics Series, Dover Publications.

Euclid. c. 300 BC, 2002. Euclid's Elements: all thirteen books complete in one volume: the Thomas L. Heath's translation, Green Lion Press.

Ewins, D.J. 2000. Modal testing: theory, practice and application, 2nd ed., Mechanical engineering research studies: Engineering dynamics series, Research Studies Press. 
Franzini, G.R., C.P.Pesce, R.T. Gonçalves, A.L.C. Fujarra, and J.R. Meneghini. 2010. An experimental investigation on frequency modulated viv in a water channel, IUTAM Symposium on Bluff Bodies Wakes and Vortex-Induced Vibrations Conference, BBVIV-6.

Franzini, G.R., A.L.C. Fujarra, J.R. Meneghini, I. Korkischko, and R. Franciss. 2009. Experimental investigation of vortex-induced vibration on rigid, smooth and inclined cylinders, Journal of Fluids and Structures 25, no. 4, 742-750. doi 10.1016/j.jfluidstructs.2009.01.003.

Franzini, G.R., R.T. Gonçalves, C.P. Pesce, A.L.C. Fujarra, C.E.N. Mazzilli, J.R. Meneghini, and P. Mendes. 2013. Vortex-Induced Vibrations Experiments with a Very Flexible Cylinder under Tension Modulation: Fourier Transform and Hilbert-Huang Spectral Analysis, Proceedings of the 15th (2013) International Symposium on Dynamic Problems of Mechanics (DINAME).

Franzini, G.R., C.E.N. Mazzilli, and C.P. Pesce. 2015. Reduced-order model for parametric excitation of a submerged, vertical and flexible cylinder: a calibration approach study, Proceedings of the 17th (2015) International Symposium on Dynamic Problems of Mechanics (DINAME).

Franzini, G.R., A.A.P Pereira, A.L.C. Fujarra, and C.P. Pesce. 2008. Experiments on VIV under frequency modulation and at constant Reynolds number, Proceedings of the 27rd (2008) International Conference on Ocean, Offshore and Arctic Engineering.

Franzini, G.R., F.R. Pereira, R.T. Gonçalves, C.P. Pesce, and C.E.N. Mazzilli. 2012. An experimental assessment of rigidity parameters of a small-scaled riser model, The Pan American Congress of Applied Mechanics (PACAM).

Franzini, G.R., C.P. Pesce, R.T. Gonçalves, A.L.C. Fujarra, and A.A.P. Pereira. 2011a. Concomitant Vortex Induced Vibration Experiments: a Cantilevered Flexible Cylinder and a Rigid Cylinder Mounted on a Leaf-spring Apparatus, Proceedings of the 14th (2011) International Symposium on Dynamic Problems of Mechanics (DINAME).

2011b. Multimodal and Mode Transition Vortex-Induced Vibrations Analysis using the Hilbert-Huang Transform Method, The 3rd International Conference on Hilbert-Huang Transform: theory and applications. Qingdao, China.

Franzini, G.R., C.P. Pesce, R. Salles, R.T. Goncalves, A.L.C. Fujarra, and P. Mendes. 2015. Experimental Analysis of a Vertical and Flexible Cylinder in Water: Response to Top Motion Excitation and Parametric Resonance, Journal of Vibration and Acoustics 137. doi 10.1115/1.4029265.

Franzini, G.R., C.P. Pesce, R. Salles, R.T. Gonçalves, A.L.C. Fujarra, and P. Mendes. 2014. Experimental analysis of a vertical and flexible cylinder in water: Response to top motion excitation and parametric resonance, Proceedings of the 33rd (2014) International Conference on Ocean, Offshore and Arctic Engineering.

Franzini, G.R., C.C.P. Santos, C.P. Pesce, and C.E.N. Mazzilli. 2016. Parametric excitation of an immersed, vertical and slender beam using reduced-order models: influence of hydrodynamic coefficients, Marine Syst Ocean Technol. doi 10.1007/s40868-016-0013-z. 
Fu, S., J. Wang, R. Baarholm, J. Wu, and C.M. Larsen. 2014. Features of Vortex-Induced Vibration in Oscillatory Flow, ASME J. Offshore Mech. Arct. Eng. 136(1):011801-011801-10. doi 10.1115/1.4025759.

Fujarra, A.L.C. 1997. Estudo em modelo reduzido de tubo flexível e liso, submetido ao fenômeno de vibração induzida por emissão de vórtices, Master's Thesis, Escola Politécnica da Universidade de São Paulo, São Paulo, Brazil. In Portuguese.

2002. Estudos Expermentais e Analíticos das Vibrações Induzidas pela Emissão de Vórtices em Cilindros Flexíveis e Rígidos, Ph.D. Thesis, Escola Politécnica da Universidade de São Paulo, São Paulo, Brazil. In Portuguese.

Fujarra, A.L.C., C.P. Pesce, F. Flemming, and C.H.K. Williamson. 2001. Vortex-induced vibration of a flexible cantilever, Journal of Fluid and Structures 15, (3-4), 651-658.

Fujarra, A.L.C., C.P. Pesce, and R. Franciss. 1997. Investigação analítico-numérica sobre a dinâmica de tubos verticalmente submersos sujeitos à ação do fenômeno de vibração induzida pela vorticidade - VIV. In Congreso Panamericano de Ingeniería Naval y Portuaria - IPIN. Montevideo, Uruguay. In Portuguese.

Fujarra, A.L.C., C.P. Pesce, and P.P.H. Parra. 1998. Vortex induced vibrations experiments on a flexible cylinder, The 8th International Offshore and Polar Engineering Conference (OMAE), pp. 393-399.

Galerkin, B.G. 1915. Rods and plates: Series in some questions of elastic equilibrium of rods and plates, Vestnik Inzhenerov i Tekhnokov 19, 897-908.

Galilei, G. 1638. Mathematical Discourses Concerning Two New Sciences Relating to Mechanicks and Local Motion,: In Four Dialogues. I. Of the Resistance of Solids Against Fractions. II. Of the Cause of Their Coherence. III. Of Local Motion, Viz. Equable, and Naturally Accelerate. IV. Of Violent Motion, Or of Projects.

Gantmacher, F. 1975. Lectures in Analytical Mechanics, Mir.

Gay Neto, A. and C.A. Martins. 2013. Structural stability of flexible lines in catenary configuration under torsion, Marine Structures 34, 16-40.

Gelfand, I.M. and S.V. Fomin. 2000. Calculus of Variations, Dover Books on Mathematics, Dover Publications. Translated from russian and edited by R.A. Silverman. Unabridged Dover (2000) republication of the translation first published by Prentice-Hall, New Jersey, 1963.

Goldstein, H. 1965. Classical Mechanics, A-W series in advanced physics, Addison-Wesley.

Gonçalves, R.T., G.R. Franzini ans C.P. Pesce, A.L.C. Fujarra, and P. Mendes. 2012. VIV experiments with a semi-immersed vertical flexible cylinder driven by top motion in a re-circulating water channel, The 65th Annual Meeting of the APS Division of Fluid Dynamics.

Gonçalves, R.T., G.R. Franzini, G.F. Rosetti, A.L.C. Fujarra, and K. Nishimoto. 2012. Analysis methodology for vortex-induced motions (VIM) of a monocolumn platform applying the hilbert-huang transform method, Journal of Offshore Mechanics and Arctic Engineering 134, no. 1, 011103-1-011103-7. 
Gonçalves, R.T., G.F. Rosetti, G.R. Franzini, J.R. Meneghini, and A.L.C. Fujarra. 2013. Two-degree-of-freedom vortex-induced vibration of circular cylinders with very low aspect ratio and small mass ratio, Journal of Fluids and Structures 39, 237-257.

Gu, J., M. Vitola, J. Coelho, W. Pinto, M. Duan, and C. Levi. 2013. An experimental investigation by towing tank on VIV of a long flexible cylinder for deepwater riser application, Journal of the Japan Society of Naval Architects and Ocean Engineers (JASNAOE) 18(3), 358-369.

Hamdan, M.N., B.A. Jubran, N.H. Shabanneh, and M. Abu-Samak. 1996. Comparison of various basic wwavelet for the analyisis of flow-induced vibration of a cylinder in cross-flow, Journal of Fluids and Structures 10, 633-651.

Hamilton, W.R. 1853. Lectures on Quaternions.

Helmholtz, H. von. 1885. On the Sensations of Tone as a Physiological Basis for the Theory of Music, Longmans, Green.

Hönig, C.S. 1978. Análise funcional e o problema de Sturm-Liouville, Edgard Blücher. In Portuguese.

Huera-Huarte, F.J. 2006. Multi-mode Vortex-Induced Vibrations of a Flexible Circular Cylinder, Ph.D. Thesis, Imperial College, London, England.

Huygens, C. 1673. Horologium Oscillatorium.

Ikoma, T., K. Masuda, and H. Maeda. 2012. Application of Wavelet Analysis for the Understanding of Vortex-Induced Vibration. Advances in Wavelet Theory and Their Applications in Engineering, Physics and Technology, Dr. Dumitru Baleanu (Ed.), InTech, Available from: http://goo.gl/RhYhLL (Retrieved: 09-26-2014).

Ilanko, S. 2009. Comments on the historical bases of the Rayleigh and Ritz methods, Journal of Sound and Vibration 319, 731-733. doi 10.1016/j.jsv.2008.06.001.

Jubran, B.A., M.N Hamdan, N.H. Shabanneh, and S. Szepessy. 1998. Wavelet and chaos analysis of irregularities of vortex shedding, Mechanics Research Communications 25, no. 5, 583-591.

Kassen, K.E. 2002. On Identification of VIV Modes from Measurements, In Proceedings of the 12th International Offshore and Polar Engineering Conference, pp. 827-833.

Kuiper, G.L. 2008. Stability of Offshore Risers Conveying Fluid, Eburon.

Lagrange, J.-L. 1989. Mécanique Analytique, Éditions Jacques Gabay. Reprint of the 1788 edition.

Lanczos, C. 1970. The Variational Principles of Mechanics, Dover Publications. Unabridged Dover (1986) republication of the 4th edition published by University of Toronto Press, Toronto, 1970.

Leissa, A.W. 2005. The historical bases of the Rayleigh and Ritz methods, Journal of Sound and Vibration 287, 961-978. doi 10.1016/j.jsv.2004.12.021. 
LIFE\&MO. 2011a. Experimentos hidro-elásticos com cilindros flexíveis - concepção e técnicas experimentais, São Paulo, Brazil. Relatório Técnico do Projeto de Dinâmica Não-Linear de Risers: Interações Não-Lineares de Natureza Hidro-Elástica e de Contato RT-1, Módulo 4. Laboratório de Interação Fluido-Estrutura \& Mecânica Offshore (LIFE\&MO) - EPUSP. Projeto de Pesquisa para a Petrobras. In Portuguese.

2011b. Experimentos hidro-elásticos com cilindros flexíveis e modelos de risers - construção, caracterização de modelos e planejamento de ensaios, São Paulo, Brazil. Relatório Técnico do Projeto de Dinâmica Não-Linear de Risers: Interações Não-Lineares de Natureza Hidro-Elástica e de Contato RT-2, Módulo 4. Laboratório de Interação Fluido-Estrutura \& Mecânica Offshore (LIFE\&MO) - EPUSP. Projeto de Pesquisa para a Petrobras. In Portuguese.

2011c. Modelagem matemática da dinâmica de risers através de técnicas de redução fazendo uso de representação por modos não-lineares, São Paulo, Brazil. Relatório Técnico do Projeto de Dinâmica Não-Linear de Risers: Interações Não-Lineares de Natureza Hidro-Elástica e de Contato RT-2, Módulo 1. Laboratório de Interação Fluido-Estrutura \& Mecânica Offshore (LIFE\&MO) - EPUSP. Projeto de Pesquisa para a Petrobras. In Portuguese.

2011d. Modelagem matemática da dinâmica de risers através de técnicas de redução fazendo uso de representação por modos não-lineares de natureza hidro-eléstica e de contato (versão revisada, com apêndices), São Paulo, Brazil. Relatório Técnico do Projeto de Dinâmica Não-Linear de Risers: Interações Não-Lineares de Natureza Hidro-Elástica e de Contato RT-1, Módulo 1. Laboratório de Interação Fluido-Estrutura \& Mecânica Offshore (LIFE\&MO) - EPUSP. Projeto de Pesquisa para a Petrobras. In Portuguese.

2011e. Modelagem no domínio do tempo com modelos fenomenológicos de VIV, São Paulo, Brazil. Relatório Técnico do Projeto de Dinâmica Não-Linear de Risers: Interações Não-Lineares de Natureza Hidro-Elástica e de Contato RT-1, Módulo 2. Laboratório de Interação Fluido-Estrutura \& Mecânica Offshore (LIFE\&MO) - EPUSP. Projeto de Pesquisa para a Petrobras. In Portuguese.

2011f. Modelagem no domínio do tempo com modelos fenomenológicos de VIV, São Paulo, Brazil. Relatório Técnico do Projeto de Dinâmica Não-Linear de Risers: Interações Não-Lineares de Natureza Hidro-Elástica e de Contato RT-2, Módulo 2. Laboratório de Interação Fluido-Estrutura \& Mecânica Offshore (LIFE\&MO) - EPUSP. Projeto de Pesquisa para a Petrobras. In Portuguese.

2012a. Experimentos hidro-elásticos com cilindro flexível vertical em canal de água cirulante (NDF), São Paulo, Brazil. Relatório Técnico do Projeto de Dinâmica Não-Linear de Risers: Interações Não-Lineares de Natureza Hidro-Elástica e de Contato RT-3.1, Módulo 4. Laboratório de Interação Fluido-Estrutura \& Mecânica Offshore (LIFE\&MO) - EPUSP. Projeto de Pesquisa para a Petrobras. In Portuguese.

2012b. Experimentos hidro-elásticos com modelo imerso de riser em catenária, sujeito à imposição de movimentos verticais no topo, São Paulo, Brazil. Relatório Técnico do Projeto de Dinâmica Não-Linear de Risers: Interações Não-Lineares de Natureza Hidro-Elástica e de Contato RT-3.2, Módulo 4. Laboratório 
de Interação Fluido-Estrutura \& Mecânica Offshore (LIFE\&MO) - EPUSP. Projeto de Pesquisa para a Petrobras. In Portuguese.

2012c. Experimentos hidro-elásticos com modelo de riser em catenária, São Paulo, Brazil. Relatório Técnico do Projeto de Dinâmica Não-Linear de Risers: Interações Não-Lineares de Natureza Hidro-Elástica e de Contato RT-3.3, Módulo 4. Laboratório de Interação Fluido-Estrutura \& Mecânica Offshore (LIFE\&MO) - EPUSP. Projeto de Pesquisa para a Petrobras. In Portuguese.

2012d. Experimentos hidro-elásticos com modelo de riser vertical, São Paulo, Brazil. Relatório Técnico do Projeto de Dinâmica Não-Linear de Risers: Interações Não-Lineares de Natureza Hidro-Elástica e de Contato RT-3.4, Módulo 4. Laboratório de Interação Fluido-Estrutura \& Mecânica Offshore (LIFE\&MO) - EPUSP. Projeto de Pesquisa para a Petrobras. In Portuguese.

Lucor, D., H. Mukundan, and M.S. Triantafyllou. 2006. Riser modal identification in CFD and full-scale experiments, Journal of Fluids and Structures 22, 905-917.

Lucor, D. and M.S. Triantafyllou. 2008. Riser Response Analysis by Modal Phase Reconstruction, Journal Offshore Mech and Arctic Engineering 130.

Mainçon, P. and C. Bernardo-Viljoen. 2013. An inverse finite element method for the analysis of VIV data, Marine Structures 33, 143-159.

Mazzilli, C.E.N. 2009. Buckling and post-buckling of extensible rods revisited: $A$ multiple-scale solution, International Journal of Non-Linear Mechanics 44, 200-208.

Mazzilli, C.E.N., S. Lenci, and L. Demeio. 2014. Nonlinear free vibrations of tensioned vertical risers, In Proceedings of the 8th European Nonlinear Dynamics Conference (ENOC2014).

Mazzilli, C.E.N., G.C. Monticelli, and N.A.G. Neto. 2011. Reduced-order modeling in non-linear dynamics: an approach based on non-linear modes, In Proceedings of the Institution of Mechanical Engineers. Part C, Journal of Mechanical Engineering Science, pp. 2354-2368.

Mazzilli, C.E.N. and C.T. Sanches. 2011. Active control of cortex-induced vibrations in offshore catenary risers: A nonlinear normal mode approach, Journal of Mechanics of Materials and Structures 6, 1079-1088.

Mclver, D.B. 1973. Hamilton's principle for systems of changing mass, Journal of Engineering Mathematics 7, no. 3, 249-261. doi 10.1007/BF01535286.

Meirovitch, L. 1980. Computational Methods in Structural Dynamics, Sijthoff and Noordhoff, Alphen aan den Rijn, The Netherlands Rockville, Md., U.S.A.

2003. Methods of Analytical Dynamics, Dover Publications. Unabridged Dover (2003) republication of the edition published by McGraw-Hill, New York, 1970.

Modarres-Sadeghi, Y., F. Chasparis, M.S. Triantafyllou, M. Tognarelli, and P. Beynet. 2011. Chaotic response is a generic feature of vortex-induced vibrations of flexible risers, Journal of Sound and Vibration 330, no. 11, 2565-2579. doi 10.1016/j.jsv.2010.12.007. 
Morooka, C.K. and R.I. Tsukada. 2011. Dynamic Behavior of Pipelines and Risers due to Vortex-Induced Vibration in Time Domain, Marine Systems and Ocean Research, $6-17$.

Morooka, C.K., R.I. Tsukada, S. da Silva, R. Franciss, and C.G.C. Matt. 2009. Model test of a steel catenary riser in a towing tank, The 28th International Conference on Ocean, Offshore and Arctic Engineering. doi 10.1115/OMAE2009-79499.

Mukundan, H. 2008. Vortex-induced vibration of marine risers: motion and force reconstruction from field and experimental data, Ph.D. Thesis, Massachusetts Institute of Technology, Cambridge, MA, USA.

Mukundan, H., F.S. Hover, and M.S. Triantafyllou. 2009. Applications od accurate VIV response reconstruction schemes, Proceedings of the 28th (2009) International Conference on Offshore Mechanics and Arctic Engineering.

Newton, I. 1687. Philosophiae Naturalis Principia Mathematica.

Pereira, F. R., R.T. Gonçalves, C.P. Pesce, A.L.C. Fujarra, G.R. Franzini, and P. Mendes. 2013. A model scale experimental investigation on vortex-self induced vibrations (VSIV) of catenary risers, Ocean, Offshore and Arctic Engineering Division. doi OMAE2013-10447.

Pereira, F. R., C.P. Pesce, R.T. Gonçalves, G.R. Franzini, A.L.C. Fujarra, R. Salles, and P. Mendes. 2012. Risers Model Test: Scaling Methodology and Dynamic Similarity, Proceedings of the 22nd (2012) international society of offshore and polar engineers.

Pereira, F.R. 2014. Investigação das vibrações induzidas pela emissão de vórtices em modelos reduzidos de riser lançados em catenária, Ph.D. Thesis, Escola Politécnica da Universidade de São Paulo, São Paulo, Brazil. In Portuguese.

Pesce, C.P. 1997. Mecânica de Cabos e Tubos Submersos Lançados em "Catenária": Uma Abordagem Analítica e Experimental, São Paulo, Brazil. Habilitation thesis. Escola Politécnica da Universidade de São Paulo. In Portuguese.

2013. Riser Dynamics: experiments with small scale models, LabOceano Ten-Years Aniversary Celebration Workshop. Rio de Janeiro, Brazil. Presentation.

Pesce, C.P., J.A.P. Aranha, and C.A. Martins. 1996. Modelo de análise da dinâmica de um SCR - Desenvolvimento de metodologia de projeto de risers rígidos em catenária. Relatório Técnico, Escola Politécnica da Universidade de São Paulo - EPUSP. Projeto de Pesquisa para a Petrobras. In Portuguese.

Pesce, C.P., J.A.P. Aranha, C.A. Martins, and M.M.O. Pinto. 1994. Análise preliminar de três risers rígidos em catenária para operação na Bacia de Campos. Relatório Técnico, Escola Politécnica da Universidade de São Paulo - EPUSP. Projeto de Pesquisa para a Petrobras. In Portuguese.

Pesce, C.P., J.A.P. Aranha, C.A. Martins, O.G.S. Ricardo, and S Silva. 1998. Dynamic curvature in catenary risers at the touch down point region: An experimental study and the analytical boundary-layer solution, International Journal of Offshore and Polar Engineering 8, no. 4, 302-310. 
Pesce, C.P. and A.L.C. Fujarra. 1997. Vibrações induzidas pela emissão de vórtices: Estimativa da vida Útil em um riser vertical. Relatório Técnico, Escola Politécnica da Universidade de São Paulo - EPUSP. Projeto de Pesquisa para a Petrobras. In Portuguese.

2000. Vortex-induced vibrations and jump phenomenon: Experiments with a clamped flexible cylinder in water, International Journal of Offshore and Polar Engineering 10, no. 1, 26-33.

Pesce, C.P., A.L.C. Fujarra, and L.K. Kubota. 2006. The Hilbert-Huang Spectral Analysis Method Applied to VIV, Proceedings of the 25th (2006) International Conference on Offshore Mechanics and Arctic Engineering.

Pesce, C.P., A.L.C. Fujarra, A.N. Simos, and E.A. Tannuri. 1999. Analytical and closed form solutions for deep water riser-like eigenvalue problem, The 9th International Ocean and Polar Engineering Conference (ISOPE).

Pesce, C.P. and C.A. Martins. 2005. Numerical Computation of Riser Dynamics. In: Chakrabarti, S.K. (Ed.) Numerical Methods in Fluid Structure Interaction, Advances in Fluid Mechanics vol. 42, WIT Press, 429 pp, Chapter 7, pgs. 253-309.

Pesce, C.P., C.A. Martins, and L.M.Y. Silveira. 2006a. Riser-soil interaction : local dynamics at TDP and a discussion on the eigenvalue and the VIV problems, Journal of Offshore Mechanics and Arctic Engineering 128, no. 1, 39-55.

2006b. Riser-Soil Interaction: Local Dynamics at TDP and a Discussion on the Eigenvalue and the VIV Problems, Journal of Offshore Mechanics and Arctic Engineering 128, 39-55.

Prado, F.S., F.Y. Sakamoto, and C.E.N. Mazzilli. 2013. An Analysis of Parametric Instability of Risers, Latin American Journal of Solids and Structures 11, 348-368.

Proakis, J.G. and D.G. Manolakis. 2007. Digital signal processing: Principles, algorithms, and applications, 4th ed., Pearson Education.

Ramani, D.V., W.L. Keith, and R.H. Rand. 2004. Perturbation solution for secondary bifurcation in the quadratically-damped mathieu equation, International Journal of Non-Linear Mechanics 39, 491-502. doi:10.1016/S0020-7462(2)00218-4.

Ramos Jr., R. and C. P. Pesce. 2003. A stability analysis of risers subjected to dynamic compression coupled with twisting, Journal of Offshore Mechanics and Arctic Engineering 125, no. 3, 183-189.

Rayleigh, Lord. 1896. Theory of sound, Dover Publications.

Ritz, W. 1909. Über eine neue methode zur lösung gewisser variationsprobleme der mathematischen physik, Journal f'ur die reine und angewandte Mathematik 135, 1-61. In German.

Rustad, A.M., C.M. Larsen, and A.J. Sørensen. 2008. FEM modeling and automatic control for collision prevention of top tensioned risers, Marine Structures 21, Issue 1, 80-112. 
Sanches, C.T., C.E.N. Mazzilli, L.D. Cunha, and C.P. Pesce. 2007. Non-linear Modal Analysis Applied to Riser Dynamics, In Proceedings of the 17th International Offshore and Polar Engineering Conference (ISOPE2007).

Sarpkaya, Turgut. 1977. In-Line and Transverse Forces on Cylinders in Oscillatory Flow at High Reynolds Number, Journal of Ship Research, 200-2016. doi 10.1016/0167-2789(83)90298-1.

Shaw, S. and C. Pierre. 1991. Non-linear normal modes and invariant manifolds, Journal of Sound and Vibration 150, 170-173.

Shaw, S.W. and C. Pierre. 1993. Normal modes for nonlinear vibratory systems, Journal of Sound and Vibration 164, 85-124.

Silveira, L.M.Y., C.A. Martins, L.D. Cunha, and C.P. Pesce. 2007. An investigation on the effect of tension variation on VIV of risers, The 26th International Conference on Ocean, Offshore and Arctic Engineering (OMAE). doi 10.1115/OMAE2007-29247.

Takafuji, F.C.M. and C.A. Martins. 2012. Comparison Between Frequency Domain and Time Domain Riser Analysis, Journal of Offshore Mechanics and Arctic Engineering 134.

Thorsen, M.J., S. Saevik, and C.M. Larsen. 2016. Time domain simulation of vortex-induced vibrations in stationary and oscillating flows, Journal of Fluids and Structures 61, 1-19. doi http://dx.doi.org/10.1016/j.jluidstructs.2015.11.006.

Tikhonov, A.N. and A.A. Samarskii. 1980. Ecuaciones de la Física Matemática, Mir Moscow. Translated from Russian to Spanish by Juán José Tolosa.

Triantafyllou, M.S. 1984. The Dynamics of Taut Inclined Cables, Quarterly Journal of Mechanics and Applied Mathematics 37. Pt. 3.

Whittaker, E.T. 1988. A Treatise on the Analytical Dynamics of Particles and Rigid Bodies, Cambridge Mathematical Library, Cambridge University Press.

Williamson, C.H.K. and R. Govardhan. 2004. Vortex-Induced Vibrations, Annual Review Fluid Mechanics 36, 313-455. 